\title{
LOW VELOCITY IMPACT ASSESSMENT OF A CARBON FIBER BICYCLE WHEEL
}

\author{
by \\ Karmanya Ratra \\ Bachelor of Applied Science, University of Waterloo (2013)
}

\author{
A thesis \\ presented to Ryerson University \\ in partial fulfillment of the \\ requirements for the degree of \\ Master of Applied Science \\ in the program of \\ Aerospace Engineering
}

Toronto, Ontario, Canada, 2019

(C) Karmanya Ratra, 2019 


\section{AUTHOR'S DECLARATION FOR ELECTRONIC SUBMISSION OF A THESIS}

I hereby declare that I am the sole author of this thesis. This is the true copy of the thesis, including any required final versions, as accepted by my examiners.

I authorize Ryerson University to lend this thesis to other institutions or individuals for scholarly research.

I further authorize Ryerson University to reproduce this thesis by photocopying or by other means, in total or in part, at the request of other institutions or individuals for scholarly research.

I understand that my thesis may be made electronically available to the public. 


\title{
LOW VELOCITY IMPACT ASSESSMENT OF A CARBON FIBER BICYCLE WHEEL
}

\author{
Karmanya Ratra
}

Master of Applied Science, Aerospace Engineering, Ryerson University, Toronto (2019)

\begin{abstract}
Carbon fiber bicycle wheels were tested under low velocity impact to monitor the damage evolution of the impact event. A wheel model designed by KQS Inc. (industrial partner) with eight different configurations, including spoke tension, number of spokes, and location of impact on the rim were investigated. IR thermography combined with PCA was used to monitor the damage during impact. Results showed that wheels in line with spokes had 16\% higher impact energy absorption compared to those impacted in between spokes on average (58.9 J vs $70.2 \mathrm{~J}$ ). The 20 spoked wheels had a slightly higher (6\%) impact energy absorption than the 24 spoked wheels. The added stiffness due to the extra spokes reduced the impact energy absorption of rim. Wheels with higher spoke tension also had slightly improved impact energy absorption (4\%). The test protocol established in this study provides a good understanding of the wheel's impact damage evolution.
\end{abstract}




\section{ACKNOWLEDGEMENTS}

This work could not be completed without the support and belief towards me from my thesis supervisor, faculty staff, and my family and friends.

I would like to thank Dr. Habiba Bougherara for her support. I am grateful for the resources she has provided me with, and the opportunities to work in her laboratories. I would also like to thank Dr. Zouheir Fawaz and Dr. Kazem Fayazbakhsh for access to their resources and providing their support.

My sincere thanks to Dr. Hamid Ghaemi for his constant feedback, valuable support, and his technical expertise. I would like to thank my peers Mr. Sadben Khan, and Mr. Jordan Kalman for their support and friendship.

I am grateful to Mr. Peter Bradley and Mr. Alan. Machin for providing their expertise whenever required and helping me with any manufacturing task.

I would like to acknowledge KQS Inc. Kevin Quan and Chris Leiphart for the opportunity to work with their products, and for the supply of bicycle wheels. The opportunity to work with them allowed me to gain valuable experience working for the cycling industry. 


\section{DEDICATION}

I would like to dedicate this thesis to my wonderful family: my fiancé, Ms. Diljot Chhina; my grandparents Dr. Sahdev and late Mrs. Raj Ratra; my parents, Dr. Navin and Mrs. Vandana Ratra; my brother, Dr. Nakul Ratra; my cousin, Mr. Vedant Khattar; my sister-in-law Kiran Chhina and my mother-in-law Mrs. Guriqbal Kaur Chhina. Thank you for your unconditional love, support, and patience throughout my journey. 


\section{TABLE OF CONTENTS}

AUTHOR'S DECLARATION FOR ELECTRONIC SUBMISSION OF A THESIS ................... ii

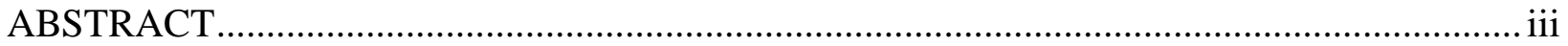

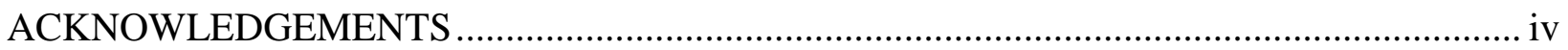

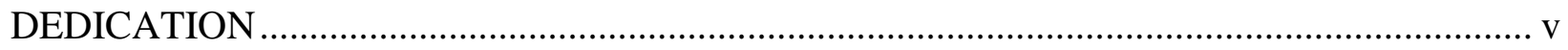

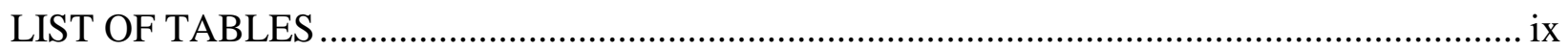

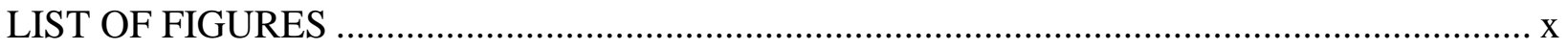

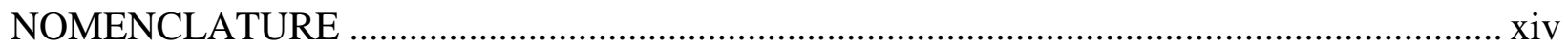

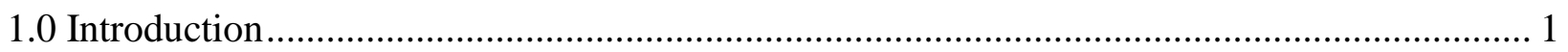

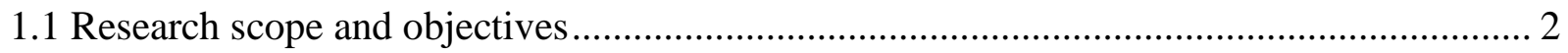

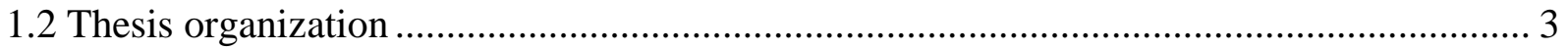

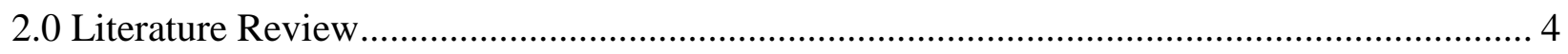

2.1 Bicycle Wheel theory .................................................................................................. 4

2.1.1 History of bicycle wheel ....................................................................................... 4

2.1.2 Forces on a bicycle wheel ................................................................................... 5

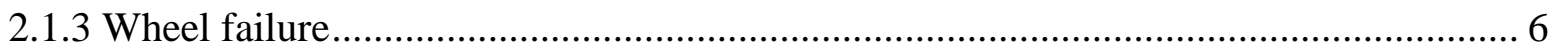

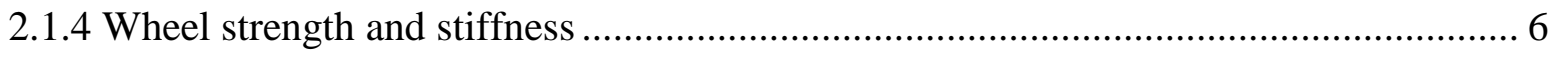

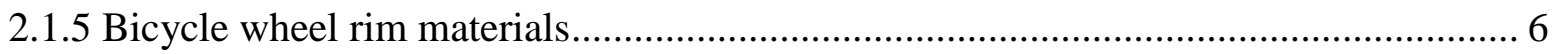

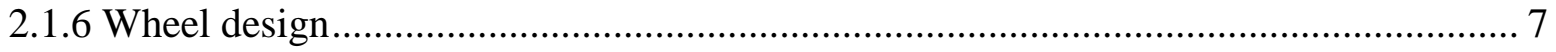

2.1.7 Radial Wheel stiffness - analytical and experimental studies ...................................... 7

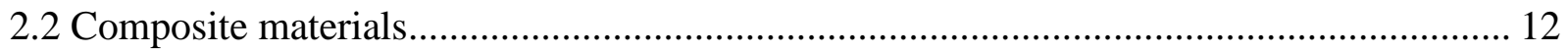

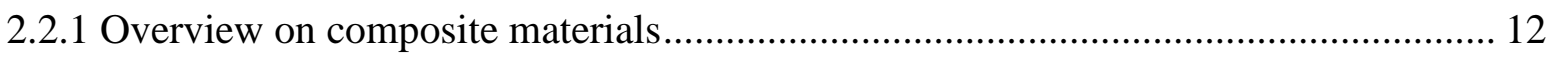

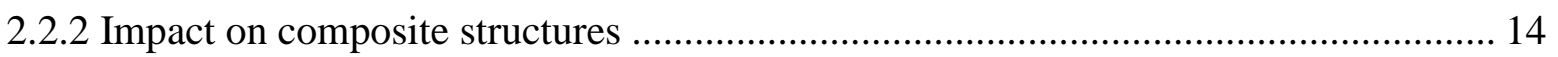

2.2.3 Low velocity impact ....................................................................................... 15

2.2.4 Composite Impact Parameters and Failure Modes ..................................................... 15 


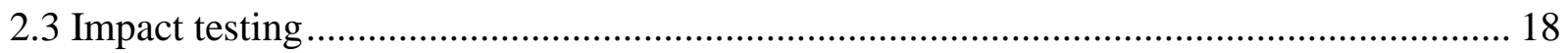

2.3.1 ASTM D7136 Low velocity impact testing for composite materials ........................... 18

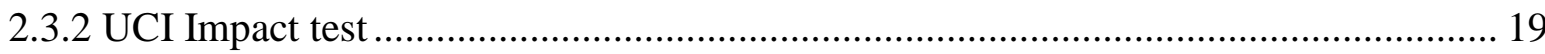

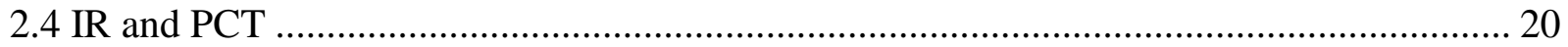

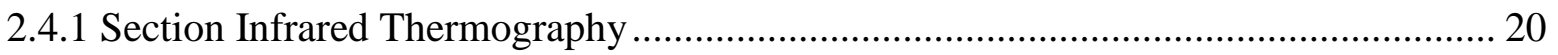

2.4.3 Active Non-Destructive Testing ........................................................................... 22

2.4.4 Post Processing - Principle Component Thermography .............................................. 23

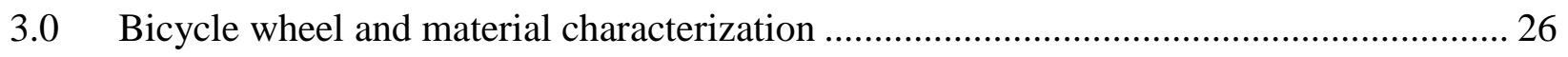

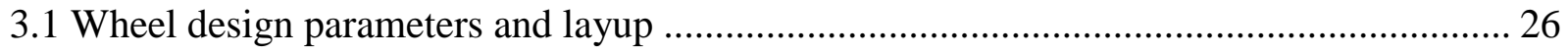

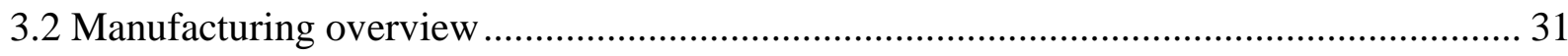

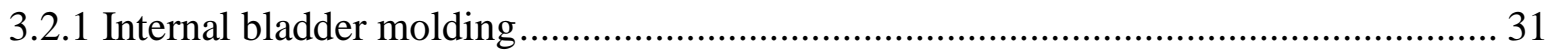

3.2.2 Manufacturing quality review ............................................................................ 32

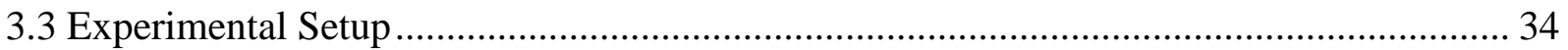

3.3.1 Design of Impact Test Apparatus ................................................................................... 34

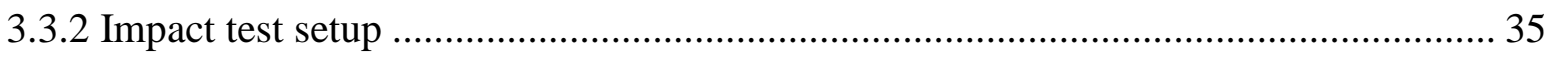

3.4 Impact testing - Design of experiment.............................................................................. 39

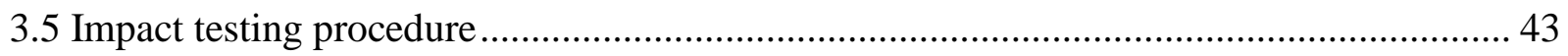

3.6 Validation of Pre-test/Post-processing Non-Destructive Inspection .................................. 45

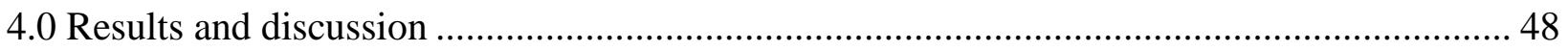

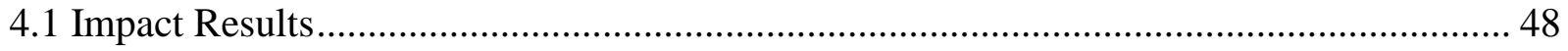

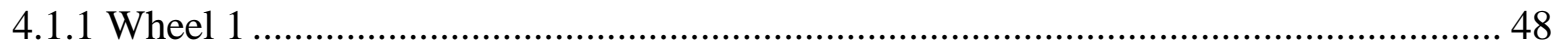

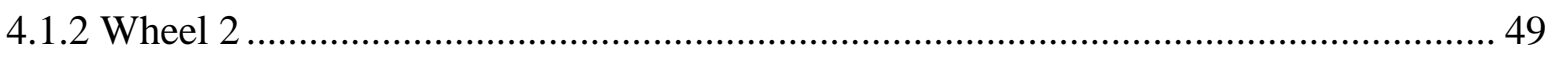

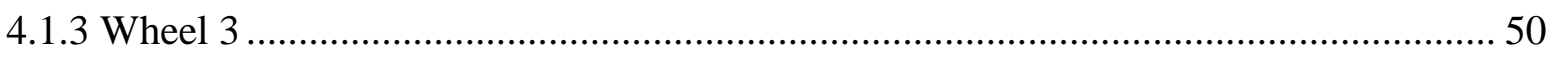

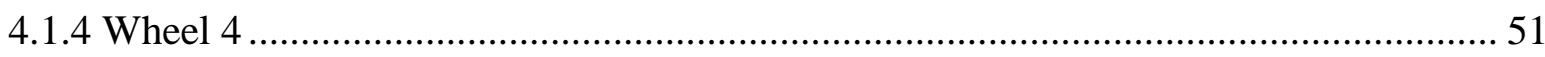

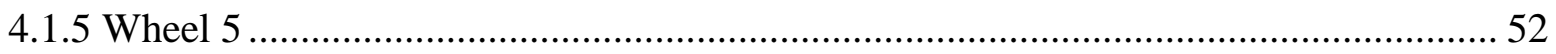


4.1.6 Wheel 6

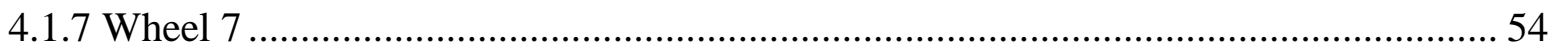

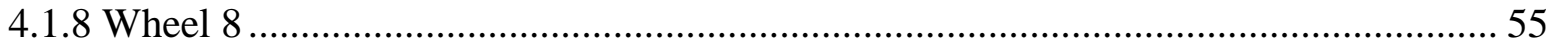

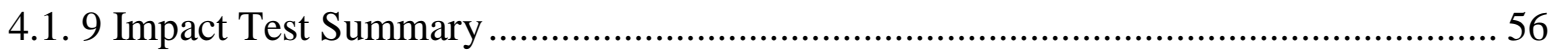

4.2 Impact Damage Assessment (Microscopy) .......................................................... 59

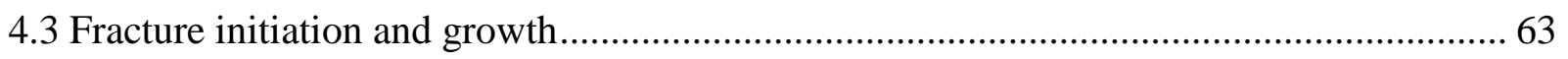

4.4 Impact Response using IR thermography ......................................................... 71

4.4.1 PCT impact damage monitoring ................................................................ 71

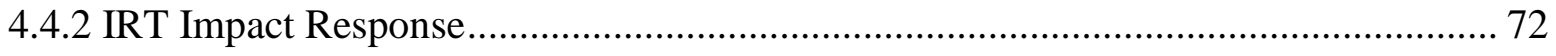

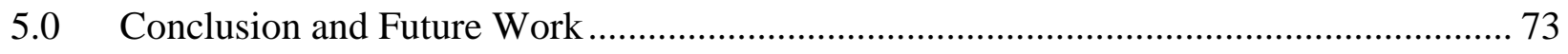

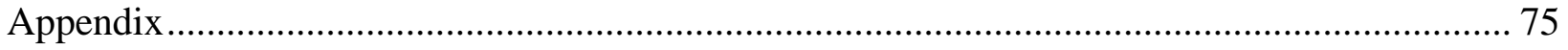

A1 Raw Data - Experimental Results........................................................... 75

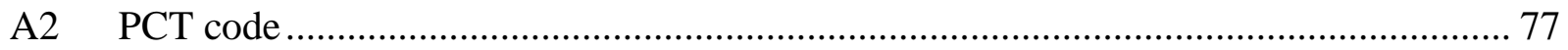

A3 Unsymmetrical laminates Modulus formulae - CLT [35] .................................... 78

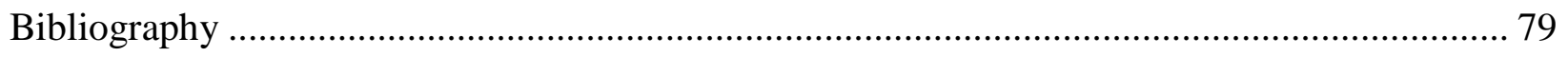




\section{LIST OF TABLES}

Table 1 - Physical specifications of wheel under test ......................................................... 27

Table 2 - Mechanical properties of T700 2510 prepeg [33] ................................................. 27

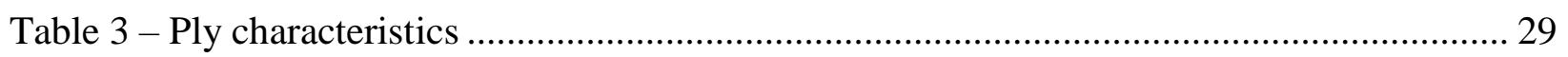

Table 4 - Homogenized membrane properties of zone-based wheel sections ........................... 30

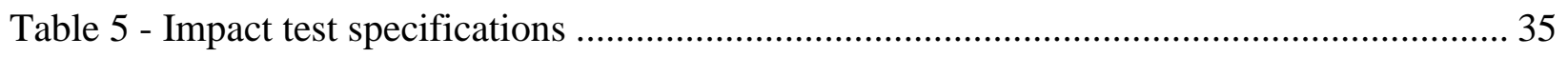

Table 6 - Jig validation - Impact velocity actual vs theoretical ........................................... 39

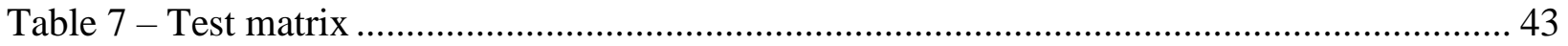




\section{LIST OF FIGURES}

Figure 1 - Anatomy of a bicycle a wheel - [2] …………..................................................... 1

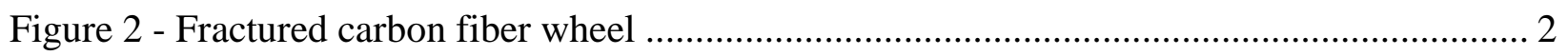

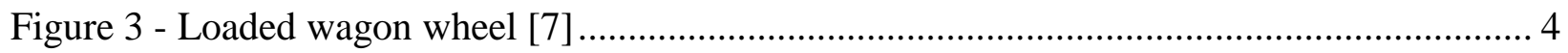

Figure 4 - Loading types on bicycle wheel [7] .................................................................... 5

Figure 5 - Spoke lacing patterns ( left to right: Radial, 2X, 3X, 4X) [7] ....................................... 7

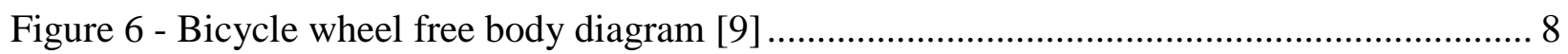

Figure 7 - Burgoyne static load test setup [4] ................................................................... 9

Figure 8 -Rim displacement [13] ...................................................................................... 11

Figure 9 - Radial structure evaluation setup by Petrone et al. [14] ............................................ 12

Figure 10 - Impact energy threshold vs ply angle [18] .............................................................. 16

Figure 11 - SEM image of prominent CFRP failure mechanisms [20] ......................................... 17

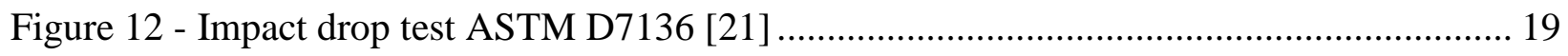

Figure 13 - UCI Wheel impact tester sample design [22] ............................................................ 20

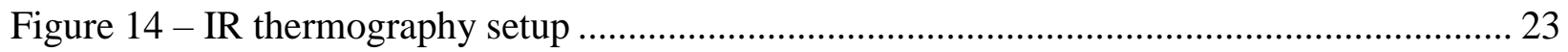

Figure 15 - Sequence of Nt image frames each with NxNy elements for Ã matrix in principal

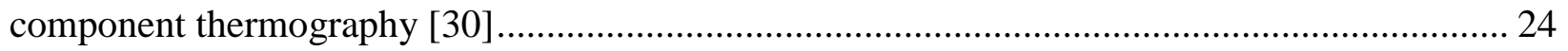

Figure 16 - Size and cross section of wheel under test ............................................................. 26

Figure 17 - Zone based and layer-based model of the wheel layup and stacking sequence......... 28

Figure 18 - Optical inspection of manufactured wheel cross section - (a) wheel cross section (b) top delamination (c) top wavy fibers and hole edge (d) wavy fibers and resin rich areas at top left corner (e) ply tapering and resin rich area - R2 (f) ply tapering and resin rich area - R3 (g) top of right bottom hole edge with micro cracks (h) bottom of left bottom hole edge with resin rich areas and delamination (i) delamination at L5 (j) new ply introduction at L5 (k) ply tapering and resin

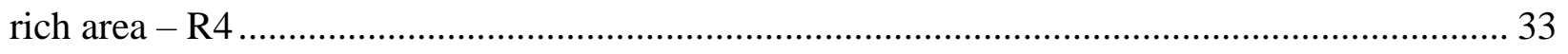

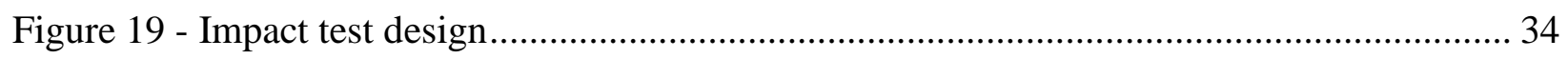

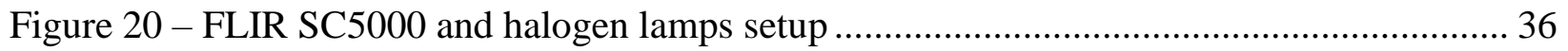

Figure 21 - High speed camera and LED illumination setup ..................................................... 37

Figure 22 - Double caliper quick release ……………...................................................... 38

Figure 23 - Spring loaded impactor stop (a) side (b) front pre-impact position (c) front post-impact position. 
Figure 24 - Design Parameters of Bicycle Wheel and Impact test ........................................ 40

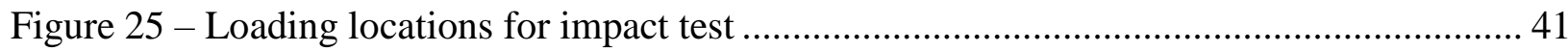

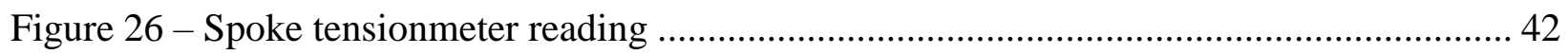

Figure 27 - Wheel truing dial gage (a) left true (b) center true (c) right true ........................... 42

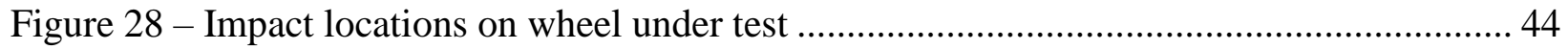

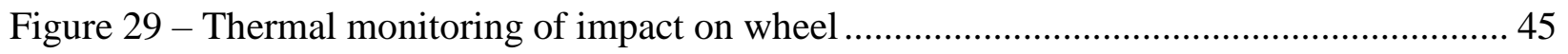

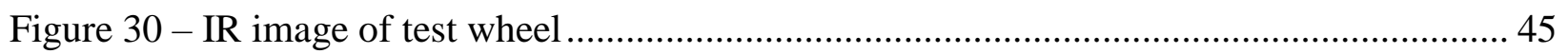

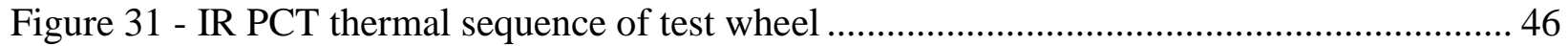

Figure 32 - (a) surface of wheel (b) PCT of surface (c) section of wheel in area of point 4 ...... 47

Figure 33 - Impact results of wheel 1 - 24 spoke - high tension - in between spokes............... 49

Figure 34 - Absorbed Energy vs Radial Deflection of wheel 1 .............................................. 49

Figure 35 - Impact Energy vs Absorbed Energy of wheel 1............................................... 49

Figure 36 - Impact results of wheel 2 - 24 spoke - low tension - in between spokes................ 50

Figure 37 - Absorbed Energy vs Radial Deflection of wheel 2 ............................................. 50

Figure 38 - Impact Energy vs Absorbed Energy of wheel 2 ............................................. 50

Figure 39 - Impact results of wheel 3 - 24 spoke wheel - high tension - in line....................... 51

Figure 40 - Absorbed Energy vs Radial Deflection of wheel 3 ........................................... 51

Figure 41 - Impact energy vs Absorbed energy of wheel 3 .............................................. 51

Figure 42 - Impact results of wheel $4-24$ spoke wheel - low tension - in line ...................... 52

Figure 43 - Absorbed Energy vs Radial Deflection of wheel 4 .......................................... 52

Figure 44 - Impact energy vs Absorbed energy of wheel 4 .............................................. 52

Figure 45 - Impact results of wheel 5 - 20 spoke wheel - high tension - in between ................. 53

Figure 46 - Absorbed Energy vs Radial Deflection of wheel 5 .......................................... 53

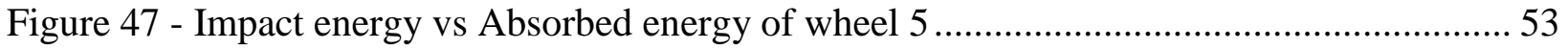

Figure 48 - Impact results of wheel 6 - 20 spoke wheel - low tension - in between ................. 54

Figure 49 - Absorbed Energy vs Radial Deflection of wheel 6 .............................................. 54

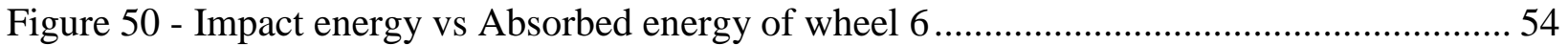

Figure 51 - Impact results of wheel 7 - 20 spoke wheel - high tension - in line ....................... 55

Figure 52 -Absorbed Energy vs Radial Deflection of wheel 7 ............................................ 55

Figure 53 - Impact energy vs Absorbed energy of wheel 7 .................................................. 55

Figure 54 - Impact results of wheel 8 - 20 spoke wheel - low tension - in line........................ 56 
Figure 55 - Absorbed Energy vs Radial Deflection of wheel 8 .................................................... 56

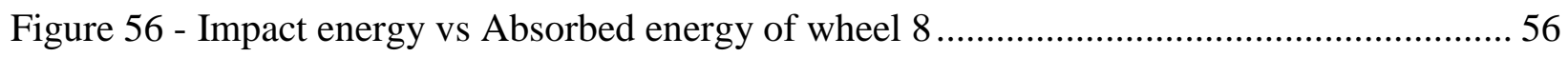

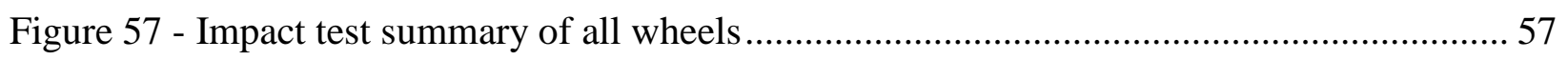

Figure 58 - Maximum rim radial displacement before failure.................................................... 58

Figure 59 - Absorbed Energy vs Radial Deflection...................................................................... 58

Figure 60 - Wheel 1 Location 1 - $33 J$ absorbed energy (a) top view - spoke hole (b) IR side view

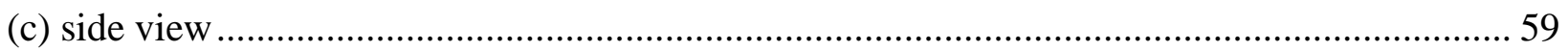

Figure 61 - Wheel 4 location 2-41 $J$ absorbed energy (a) top view - spoke hole (b) IR side view

(c) side view.

Figure 62 - Wheel 8 location 3-46 J absorbed energy (a) top view - spoke hole (b) IR side view

(c) side view. 60

Figure 63 - Wheel 7 location 1 - $51 J$ absorbed energy(a) top view - spoke hole (b) IR side view (c) side view. 61

Figure 64 - Wheel 7 location 2-61 J absorbed energy (a) top view - spoke hole (b) IR side view (c) side view. 61

Figure 65 - Wheel 7 location $374 J$ absorbed energy (a) top view - spoke hole (b) IR side view

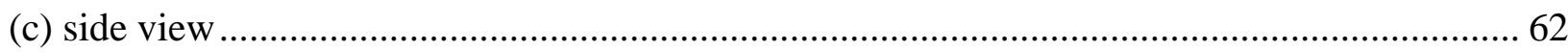

Figure 66 - Average crack length vs energy absorbed of each test ............................................ 63 Figure 67 - Section cuts on (a) Wheel 1 (b) Wheel 4 (c) Wheel 8 (d) Wheel 7 location 1 (e) Wheel

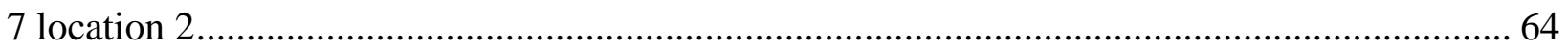
Figure 68 - Wheel 1 location 1 - $33 J$ absorbed - (a) cross section (b) layer separation at TM (b) layer separation at TR (c) microcracks and delamination TR1 (d) delamination inR1 (e)(f) delamination in L3/L4 (g) right cured zone R4/R5 (h) wavy fibers in L4/L5 ............................. 65 Figure 69 - Wheel 4-41 $J$ absorbed energy - (a) cross section (b)fracture at L1 (c) delamination and layer separation in TM/TR/TR1 (d) delamination in R2 (e) delamination in R3 (f) fiber fracture in R3/R4 (g) delamination in R4 (h) delamination in R5/BR (i) ply fracture in L4/L5 (j) ply frature

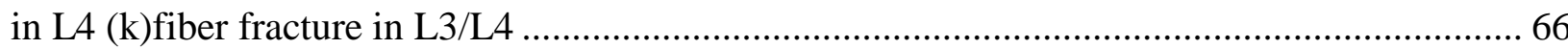
Figure 70 - Wheel 8 - $46 J$ absorbed energy - (a) cross section (b) delamination and fracture in $\mathrm{TL} / \mathrm{TM}$ (c) fiber fracture in TM/TR/TR1 (d) delamination and fracture in R3/R4 (e) delamination in R4 (f)fiber fracture in R4/R5 (g) fiber fracture in L4/L5 (h) fiber fracture in L3/L4 (i)

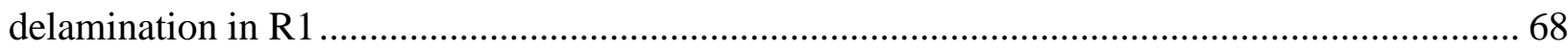


Figure 71 - Wheel 7 - location 1 - $51 J$ absorbed energy - (a) cross section (b) delamination across $\mathrm{L} 2 \rightarrow \mathrm{TM}$ (c) delamination across $\mathrm{TM} \rightarrow \mathrm{R} 2$ (d) delamination and fragmentation across $\mathrm{R} 2 \rightarrow \mathrm{R} 4$ (e) delamination in R5 (f) delamination in BR (g) delamination in BL (h) fiber fracture and delamination across $\mathrm{L} 2 \rightarrow \mathrm{L} 4$. 69

Figure 72 - (a) cross section (b) fracture and fragmentation across L2 $\rightarrow$ TM (c) delamination across $\mathrm{TM} \rightarrow \mathrm{TR} 1$ (d) delamination and fracture across TR1 $\rightarrow \mathrm{R} 2$ (e) delamination in R2 (f) fiber fracture in R3/R4 (g) delamination in R5 (h) delamination in BL (i) delamination and fiber fracture in L4/L5 (j) delamination and fracture in L3/L4 (k) fracture and fragmentation in R2/R3 .......... 70

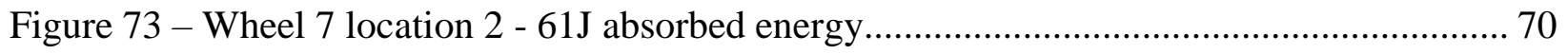

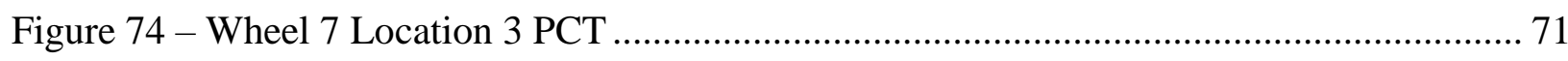

Figure 75 - Temperature profile of wheel 7 location 3 - 74J absorbed energy ......................... 72

Figure 76 - Change in surface temperature from absorbed energy in failed specimens ............. 72 


\section{NOMENCLATURE}

\begin{tabular}{|c|c|c|c|}
\hline $\mathrm{V}_{\mathrm{f}}$ & Fibre volume fraction & $\mathrm{V}_{\mathrm{m}}$ & Matrix volume fraction \\
\hline $\mathrm{E}_{\mathrm{f}}$ & Elastic modulus of fibre & $\mathrm{E}_{\mathrm{m}}$ & Elastic modulus of matrix \\
\hline$E_{11}^{T}$ & $\begin{array}{l}\text { Long. elastic modulus } \\
\text { (tension) }\end{array}$ & $E_{11}^{C}$ & $\begin{array}{l}\text { Long. elastic modulus } \\
\text { (compress.) }\end{array}$ \\
\hline$E_{22}^{T}$ & $\begin{array}{l}\text { Trans. elastic modulus } \\
\text { (tension) }\end{array}$ & $E_{22}^{C}$ & $\begin{array}{l}\text { Trans. elastic } \\
\text { (compress.) }\end{array}$ \\
\hline $\mathrm{G}_{12}$ & In-plane shear modulus & $v$ & Poisson's ratio \\
\hline$\gamma_{22}^{\mathrm{U}}$ & In-plane shear strain & $\mathrm{t}$ & Laminate thickness \\
\hline $\mathrm{E}_{\mathrm{a}}, \mathrm{E}_{\mathrm{absorbed}}$ & Energy absorbed & $\mathrm{E}_{\text {rebound }}, \mathrm{E}_{\mathrm{r}}$ & Rebound energy \\
\hline Eimpact $_{\text {in }}$ & Impact Energy & $\mathrm{m}$ & Impact mass \\
\hline$h_{i}$ & Initial height & $\mathrm{h}_{\mathrm{r}}$ & Rebound height \\
\hline $\mathrm{V}_{\mathrm{i}}$ & Impact velocity & $\mathrm{V}_{\mathrm{r}}$ & Rebound velocity \\
\hline $\mathrm{g}$ & Acceleration due to gravity & $\Delta \mathrm{T}$ & Change in Temperature \\
\hline $\mathrm{P}$ & Load & $\mathrm{M}_{0}$ & Rim Bending Moment \\
\hline $\mathrm{H}_{0}$ & Rim Tangential Force & $\mathrm{V}_{0}$ & Rim Radial Shear force \\
\hline$\phi$ & $\begin{array}{l}\text { Angle between spoke and } \\
\text { applied load }\end{array}$ & $\theta$ & Angle between spokes \\
\hline $\mathrm{T}_{\mathrm{i}}$ & Tension of spoke & $\mathrm{E}_{\text {rim }}$ & Elastic modulus of the rim \\
\hline ERD & Effective rim diameter & $\mathrm{D}$ & Rim diameter \\
\hline$A_{\text {rim }}$ & Cross sectional rim area & $\mathrm{J}$ & Rim Polar Moment of Inertia \\
\hline $\mathrm{I}_{\mathrm{xx}}$ & Moment of Inertia about $\mathrm{x}$ axis & $\mathrm{I}_{\mathrm{yy}}$ & Moment of Inertia about y axis \\
\hline $\mathrm{K}_{\text {spring }}$ & Spring equivalent of spokes & $\mathrm{n}$ & Number of spokes \\
\hline $\mathrm{E}_{\mathrm{s}}$ & Elastic modulus of spokes & $\mathrm{A}_{\mathrm{s}}$ & Cross sectional area of spokes \\
\hline
\end{tabular}




\begin{tabular}{|c|c|c|c|}
\hline 1 & Length of spokes & $\alpha$ & Angle between spoke and hub \\
\hline A & Principal matrix & $\tilde{A}$ & Standardized principle matrix \\
\hline $\mathrm{n}_{\mathrm{x}}$ & Number of pixels per row & $\mathrm{n}_{\mathrm{y}}$ & Number of pixels per column \\
\hline M & Number of rows & $\mathrm{N}$ & Number of columns \\
\hline$\mu_{\mathrm{n}}$ & Mean & $\sigma_{\mathrm{m}}^{2}$ & Variation per pixel \\
\hline $\mathrm{U}$ & Matrix of orthogonal modes & $\Gamma$ & Matrix of singular values \\
\hline EN & $\begin{array}{l}\text { Infrared thermography } \\
\text { Non-destructive evalua } \\
\text { Principal component th } \\
\text { Finite element analysis }\end{array}$ & phy & \\
\hline
\end{tabular}




\section{Chapter 1}

\subsection{Introduction}

In the high-performance sporting goods industry, composite materials have become increasingly indispensable. Carbon fiber's excellent mechanical properties, low weight, and design flexibility has not only made it more desirable for users, but facilitated large investments for research among industry and academia [1]. Since composites are relatively new compared to its metallic counterparts, new materials, development processes, and manufacturing methods are being established to ensure they exceed metals in all performance criterion.

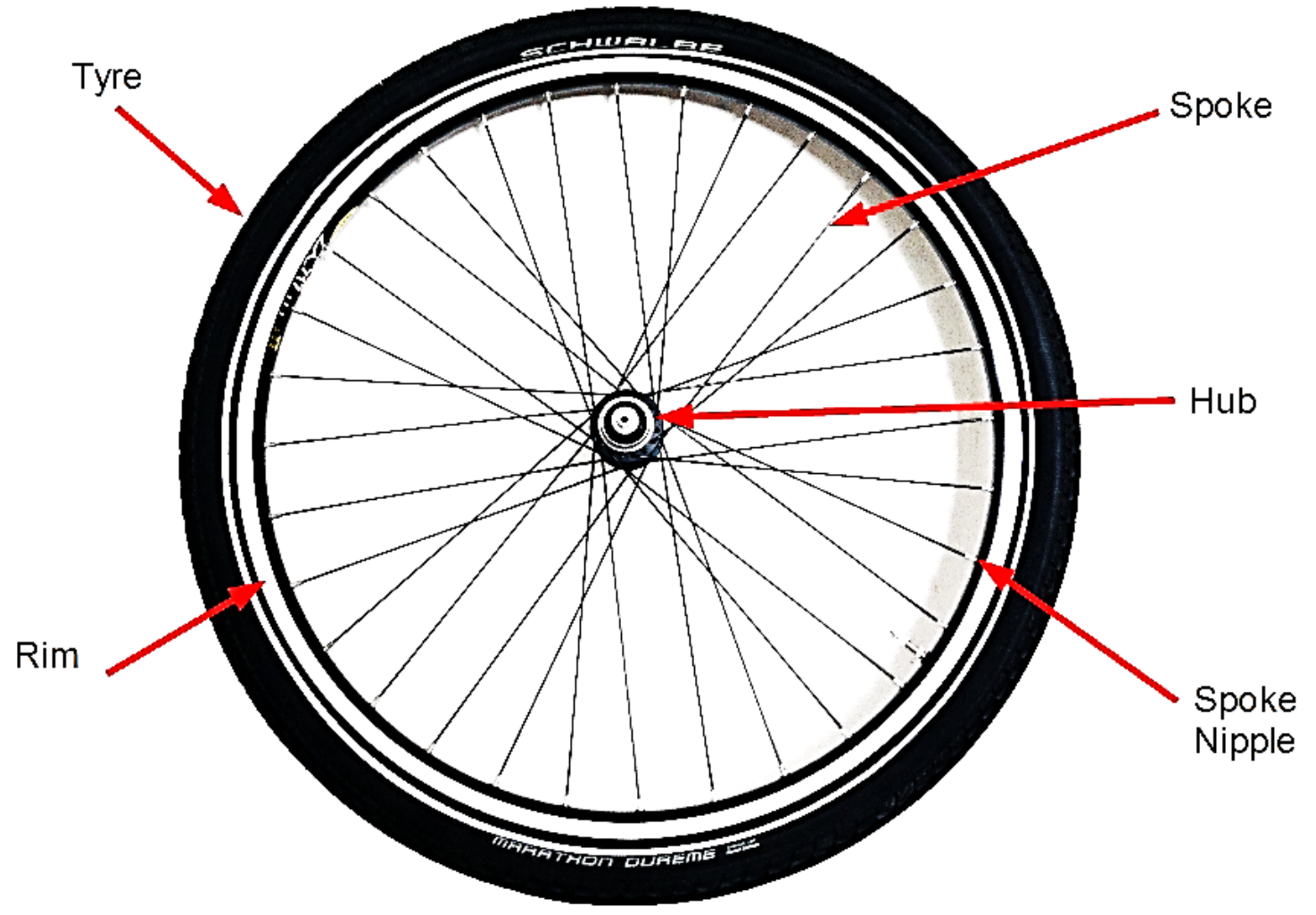

Figure 1 - Anatomy of a bicycle a wheel - [2]

Composite materials, including carbon fiber composites, have been used to build bicycle rims since the early 2000s due to its superior performance over aluminum and steel. Its excellent strength to weight ratio, ensures that with minimal weight its performance maintains a specific standard. Due to its dampening properties, it absorbs vibrations better than metal which makes it more comfortable for the rider [3]. Its design tailor-ability allows deep cross sections which can 
add structural and aerodynamic advantages. A bicycle wheel has almost 400:1 strength to weight ratio, making it one of the most efficient structures ever designed. This is achieved by pre-stressing the wheel in three different ways [4]. This includes:

1. The pressure in the tire helps dampen and spread out the forces over a larger area [4].

2. The bicycle rim is considered a medium for force transfer between the ground and the central hub [4].

3. The spokes, which are attached to the middle flange help, provide majority of the lateral, radial, and torsional resistance [4].

Despite the advantages of composites, the higher costs associated with their manufacturing processes limited their use for high end market [5]. In addition, problems related to the appearance of cracks in the composite wheel upon impact created a challenge to the cycling industry which resulted in the conclusion of the wheel being classified as 'strong but brittle'. These cracks are produced instantly upon a single impact and not among repeated cyclic loadings. This cracks also have been seen in situations where cyclists are traveling at higher velocities and sudden wheel failure can occur, compromising the rider's safety.

\subsection{Research scope and objectives}

An industry partner was observing from the design and manufacturing of its high-performance bicycle wheels, impact related damage during field use. The wheels are composed of aerospace grade carbon fiber laminates (T700/YPH200) and designed for professional racing. Figure 2 shows a wheel specimen provided by the industry partner in which the rim has fractures propagating radially from the top of the rim to the bottom, and tangentially across the rim.

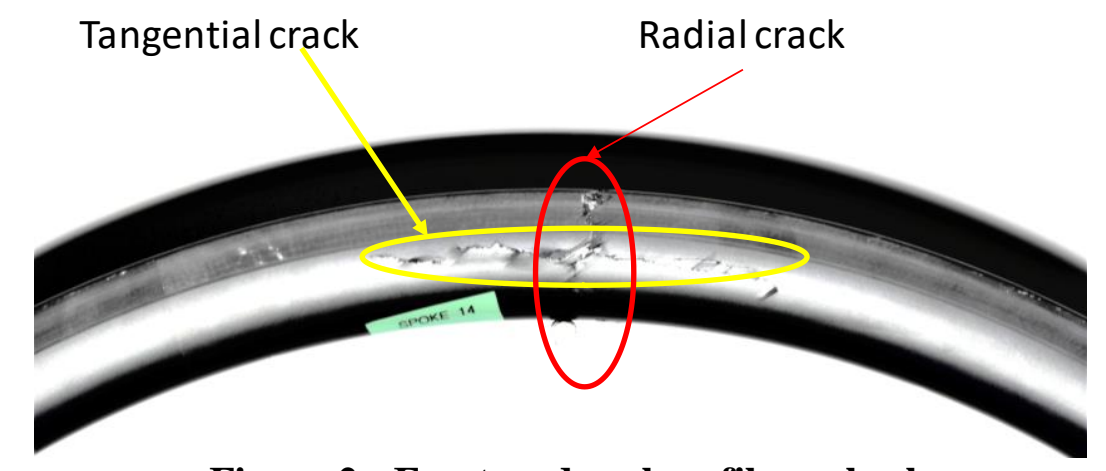

Figure 2 - Fractured carbon fiber wheel 
These cracks would be unsafe during a ride as there is a high chance of a catastrophic failure leading to injury. The company did not understand the cause and the origin of these impact failures which may be due to several parameters, such as wheel design, material and manufacturing process used. Therefore, the main objective of this research project is to evaluate the impact performance of this composite wheel and provide recommendation for design improvement to the company. Specifically, this study will focus on low velocity/low energy impact loading of a single bicycle rim design under different wheel configurations. The impact tests will be performed on the same rim design with eight different variations with respect to spoke tension, the number of spokes, and the impact location. The impact performance of the composite rim is evaluated based on its energy absorbing capacity, maximum radial deflection, damage initiation and propagation.

To perform the impact tests, a drop-tower type impact apparatus with $12.432 \mathrm{~kg}$ weight was designed and manufactured in-house. An infrared thermography camera was used to inspect the wheels before and after impacts, and to monitor the rim's external surface thermal energy dissipation during the impact. In addition, an image processing algorithm, known as Principle Component Thermography (PCT), was adopted in order to enhance the IR images so that it can show small cracks on and underneath the surface of the wheel [6].

\subsection{Thesis organization}

This thesis report includes three chapters in addition to the introduction and the conclusion. Chapter 2 is a literature review which is split up into 3 sections. The first section is a summary of the analytical and experimental studies conducted that investigate the mechanics of a bicycle wheel; the second section provides an overview of composite materials and impact testing protocols; and the third section describes the use of infrared thermography as a method for nondestructive evaluation in a composite structure. Chapter 3 begins with the physical, material and manufacturing characterization of the wheel being tested; and is followed by the design of the impact apparatus, the test setup parameters, and the testing procedure used to conduct the experiment. Chapter 4 reviews the results of the experiment and discusses the impact performance of the wheel. The report is then concluded with an investigation of the fracture mechanics in the rim cross-section. 


\section{Chapter 2}

\subsection{Literature Review}

\subsection{Bicycle Wheel theory}

\subsubsection{History of bicycle wheel}

The wheel can be defined as a load-carrying device that enables motion. It is one of the fundamental mechanisms behind many complex machines. The bicycle wheel consists of a rim, a hub, and spokes connecting the hub to the rim at equal angular displacements. An axle goes through the hub, connecting the wheel to the fork and the frame of the bicycle. In the traditional wooden bicycle wheel, the normal force is transmitted from the ground to the hub by compression of the bottom spokes [7].

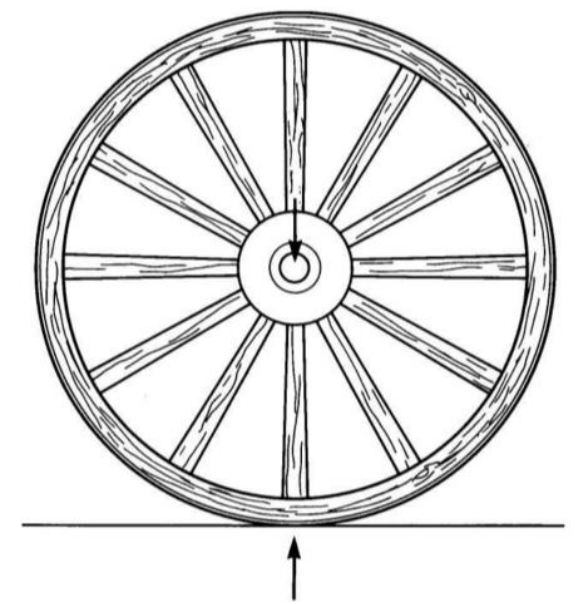

Figure 3 - Loaded wagon wheel [7]

In 1871, James Starley introduced the concept of pre-tensioned wire steel spokes and replaced the compression based wooden predecessors [7]. This design allowed the wheel to exceed the radial stiffness of the wooden wheel and decrease its weight. Starley discovered that the buckling limit of the wire spokes was proportional to the pretension applied, as long as the prestress was within the elastic limit of the steel. The force to buckle a pretensioned wire steel spoke is far greater than the buckling limit of the solid wooden spoke. In his first design, the spokes were in a radial pattern connected to the center of the hub. In 1874, Starley enhanced the design to have spokes inclined to either side of the hub to add lateral stiffness [7].

In 1888, Dunlop invented the pneumatic tire system which consisted of an air-filled rubber tube enclosed by an exterior fabric and rubber layer. The loop was attached to the outer perimeter 
of the wheel. The inflated tire helped to dampen the stresses on the rim by spreading the load over a larger area, and in doing so provide added comfort to the rider [7].

Since then, although new materials, processes and techniques have been implemented to optimize the performance of the wheel, the overall design of the bicycle wheel has stayed consistent. Studies over time have explained in more detail why the wheel is as efficient as it is. The modern bicycle wheel has proven to be an elegant structure capable of handling more than a hundred times its own weight [8].

\subsubsection{Forces on a bicycle wheel}

The bicycle wheel supports a variety of static and dynamic loads. Loads including spoke tension and tire inflation are known to be static because they remain constant. Dynamic loads can be applied laterally, tangentially, and radially. The weight of the cyclist, frame, and the flatness of the road cause radial loads as it displaces the portion of the rim in contact with the ground towards the hub [8]. Impact loading such as jumps, and uneven terrain can create high radial and lateral forces resulting in large spoke deformation. Tangential or torsional loads can occur through braking or in the rear wheel with the chain turning the sprockets. Road friction can also cause tangential loading.

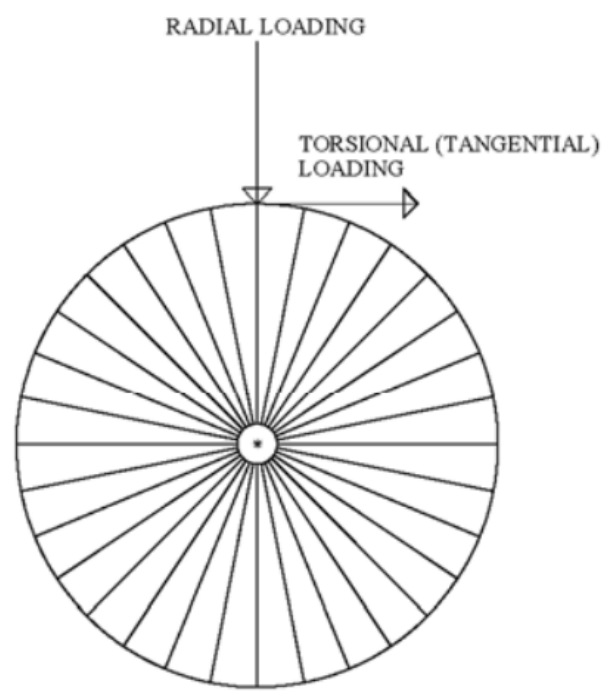

SIDE VIEW

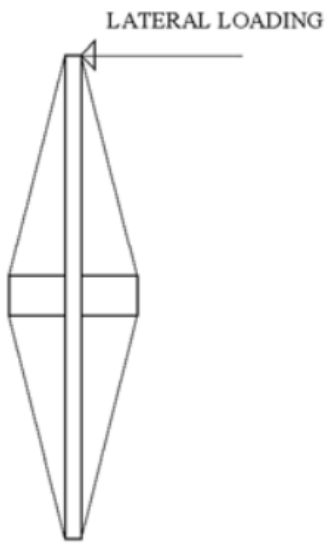

FRONT VIEW

Figure 4 - Loading types on bicycle wheel [7] 


\subsubsection{Wheel failure}

Wheel failure is most often attributed to spoke fatigue and spoke misalignment. When there is spoke fatigue, the spoke gradually loosens resulting in a decrease in its ability to withstand radial and lateral loads [7]. Sudden collapses or crushing can also occur due to excessive loading. When facing these high radial and lateral loads, the spokes will lose its pretension and buckle. The excess energy from the impact is then concentrated into the rim and if it exceeds the stress threshold of the rim material, a crack will initiate. Depending on the extent of the load and the material properties of the rim, the rim may be able to spring back and return the spokes to its pretensioned state [7].

\subsubsection{Wheel strength and stiffness}

There are many factors that determine the strength of a wheel including the rim and spoke materials, the physical characteristics of the rim, spoke diameter, spoke patterns, and spoke length [8]. In general, a strong wheel is one with a large cross section and spokes in high tension. The radial strength of the wheel is the load at which the spokes lose their spoke tension [7]. The compressive strength of the rim is defined as the maximum compressive hoop stress the rim can withstand before saddling.

Radial stiffness is a measure of force required to displacement the rim and spokes radially. Spoke length, material of the rim, and cross-section moment of inertia all affect this parameter. Lateral stiffness is the wheel's ability to withstand lateral displacement. Parameters that affect this parameter include flange spacing, rim strength, rim cross-section; and the number, material, and thickness of the spokes.

\subsubsection{Bicycle wheel rim materials}

Rims are made in various materials, including aluminum, steel, and carbon fiber composites. Aluminum is a material of choice for general wheels due to its manufacturability, strength to weight ratio, and thermal conductivity. Steel requires very thin walls so that it can compare to aluminum in weight, and economically it is a very good choice. Carbon composites are used for high performance applications where there is a need for specialized aerodynamic cross sections, optimized spoke holes, and light weight [3]. 


\subsubsection{Wheel design}

Conventional wheel building is the process of selecting the best components and configuration for the specific application. After designing the rim, the number of spokes, spoke tension and spoke pattern need to be defined while ensuring compatibility with off-the-shelf hubs. The different spoke patterns provide a trade-off between radial strength and tangential strength. The most common spoke patterns are radial, $1 \mathrm{X}, 2 \mathrm{X}, 3 \mathrm{X}$, and $4 \mathrm{X}$. In general, the more crosses result in higher tangential strength [7]. A cross is the number of times a spoke crosses over another spoke and can be achieved by ensuring the spoke is installed on a hub spoke hole that is not radially in line with the rim spoke hole. The radial pattern should never be used for a rear wheel as it has no support for tangential loading, however it can be used on front wheels to provide high impact resistance [7].

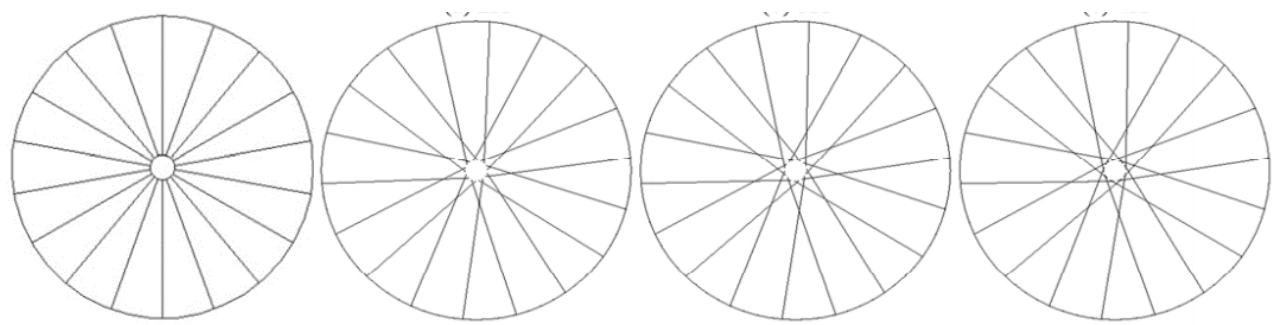

Figure 5 - Spoke lacing patterns ( left to right: Radial, 2X, 3X, 4X) [7]

\subsubsection{Radial Wheel stiffness - analytical and experimental studies}

The radial stiffness of a wheel $k$, can be used to estimate the force per unit required to displace the rim. It has been modeled analytically with different theories and tested over the last 100 years. The models and experiments have considered a combination of spoke material, length, number of spokes, pretension, spoke angles, pattern, rim radius, rim cross section as input parameters.

One of the first and most prolific series of academic studies performed on analyzing the stresses in a bicycle rim were done by Alfred J. S. Pippard. Pippard's method was to treat the wheel as a set of redundant members in which by sectioning the wheel, the model could be simplified to a hub with 2 spokes and a rim section [9]. In this model, (Figure 6) if a load (P) was applied anywhere across the simplified section, the spokes represent radial supports and the ends of the 
rim section have a bending moment $\left(\mathrm{M}_{0}\right)$, a tangential force $\left(\mathrm{H}_{0}\right)$, and a radial shear $\left(\mathrm{V}_{0}\right)$. The strain energy theorem can then be applied to find a static equilibrium and solve for the associated stresses.

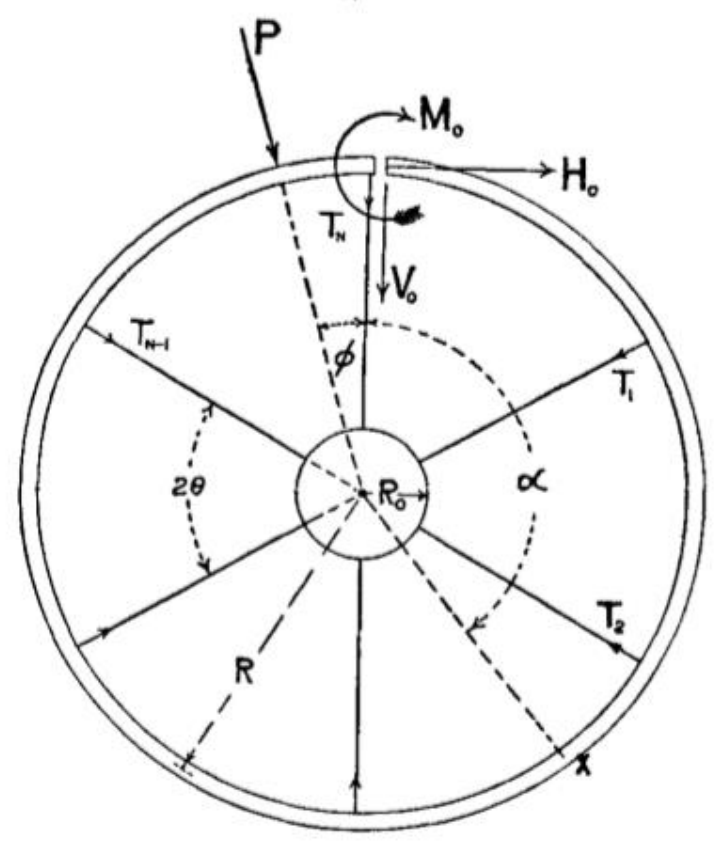

\section{Figure 6 - Bicycle wheel free body diagram [9]}

Pippard's model for the radial stiffness includes the variables radius of the rim, the modulus and cross-sectional moment of inertia of the $\operatorname{rim}\left(E_{R} I_{z Z}\right)$, the cross-sectional area of the spoke, modulus of the spoke material, and the length of the spoke. The study considers cases with a rigid and non-rigid rim, and when the applied load on the rim was either in line with the spokes or in the middle of the two spokes. It was found that when the applied load was in line with the spokes, the maximum compressive stress was calculated. When the load was applied in between the spokes, the maximum bending moment was calculated [9]. To verify his analytical model a test was performed consisting of a wheel with instrumented spokes loaded with a calibrated weight. The study found a good agreement as the maximum error between estimated and measured spoke loads were $13 \%$. However, Pippard's analysis was limited as it did not consider the curvature of the rim, the compression of the rim due to the spokes pre-tension, and the lateral shear [9].

Hetenyi et al. [10] built on Pippard's model by applying the concepts of a curved beam on an elastic foundation. Using this theory, practical models of radial deflection, bending moment, tangential and shear forces were developed which could include parameters such as number of 
spokes (which extended the model for a full wheel instead of sectioning), effective length of the spokes, and the curvature of the rim. Hetenyi showed analytically that if an initial tension is applied to the spokes, the structure can resist compressive and tensile forces, and the force produced by the pretension of the spokes on the rim will be proportional to a radial displacement of the rim i.e. hoop compression [10].

Whitt and Whilson [8] concluded: "Under load, a spoked wheel takes up not an oval shape, as is often stated, but an approximately circular shape with a flattened portion in the vicinity of road contact. In the spoke wheel the increased compressive stress in the rim increases the tension of all spokes except those in the (slightly) flattened region, where the spoke tension naturally decreases (Figure 7). The load on the axle is then relieved by the combined effect of the increased spoke tension at the top of the wheel and the decreased tension in the region of contact. All other spokes have approximately equal tension and balance each other [8]."

Burgoyne and Dilmaghanian [4] tested Pippard's theories and validated Whitt et al. [8] observation by demonstrating how pre-stressing was the key element in building an efficient bicycle wheel. They did a variety of tests in which they instrumented 4 spokes and monitored its strain as the wheel revolved. The setup consisted of the wheel attached to a rigid frame, through a horizontal stabilizing arm attached to its hub axle. A loading tie was also connected to the axle vertically and the applied load was adjusted with a screw jack. Figure 8 below illustrates the custom setup in this test.

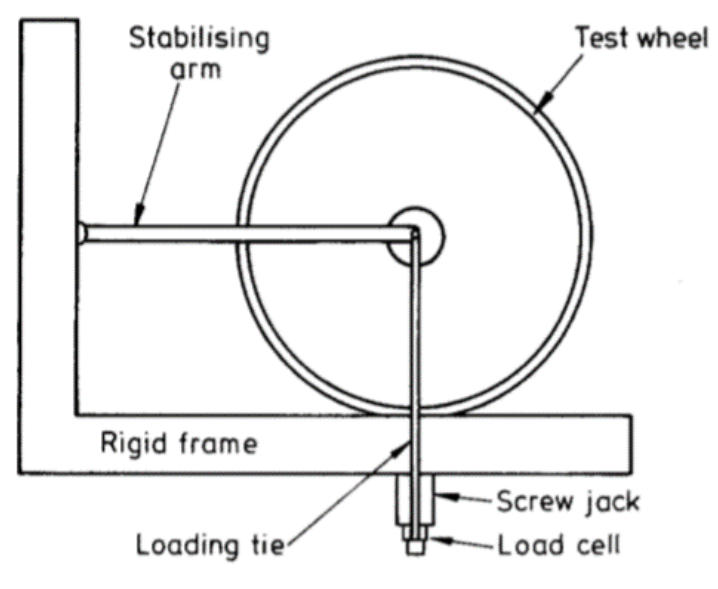

Figure 7 - Burgoyne static load test setup [4] 
Burgoyne et al. [4] proved that Pippard's analysis was valid for the case of the bare rim and proved the models of a straight beam rested on a uniformly distributed elastic foundation was in good agreement, and thus could work as a simplified approximation. In this test the influence of the tire was apparent as the peak stress on the spoke was reduced. By assuming a uniformly distributed applied load over a short length, the influence of the tire could be accurately modeled.

Gavin et al. [11] studied the effects of spoke pattern on wheel stiffness and spoke fatigue. They discovered that spoke pattern has a minimal effect on the radial stiffness of the wheel; however the more crosses in the spoke pattern, the higher the lateral stiffness [11]. They compared analytic models such as Hetenyis, to a 3-dimensional frame analysis model of the rim-spoke-hub system. The frame analysis included out-of-plane bending, radial and torsional deformation of the rim, and bending deformation of the spokes. The loading tests involved in this study included static compression loading ranging from $200 \mathrm{~N}$ to $450 \mathrm{~N}$, and live road tests. Gavin assumed a rigid connection between spokes and rim/hub due to high friction. Monitoring spoke strains during his loading tests, he found consistency among the $2 \mathrm{X}, 3 \mathrm{X}, 4 \mathrm{X}$ laced wheel sets. He found that since out-of-plane bending stiffness and torsional stiffness are coupled in curved beams, they would require two different independent measurements [11].

Minguez et al. [12] carried out an analysis for the behavior of a spoked bicycle wheel when loaded at the hub axle and supported at the rim. This study developed important contributions by including the influence of spoke pretension. The stiffness of the wheel is calculated based on ratios from hub and rim displacement, rim's moment of inertia, cross-sectional area of the spokes, and the modular ratios of the rim/spoke materials. It was found that the rim cross-sectional moment of inertia and the number of spokes influence the rim stiffness the largest. Although reducing the number of spokes will lower the wheel radial stiffness, increasing the individual pretension of a spoke can help recover a fraction of the losses [12]. If there are too many spokes in the wheel, the rim bending stiffness is too high and this can concentrate stress from an applied load within the rim before the spokes are out of pretension [12]. 
Ford extends that the accepted models of radial deflection are limited as they do not consider the lateral and torsional reactions of the spokes or rim cross section [13]. By redefining rim displacement (Figure 8), into the rim is subject to in-plane bending (v), out-of-plane bending $(\mathrm{u})$, and twist $(\phi)$ [13]. The rim is modeled initially as a circular beam with a constant cross-section, where the shear center and centroid coincide. Ford concludes that knowledge of stress distribution under large loads could reveal how plastic deformation and buckling occurs [13].

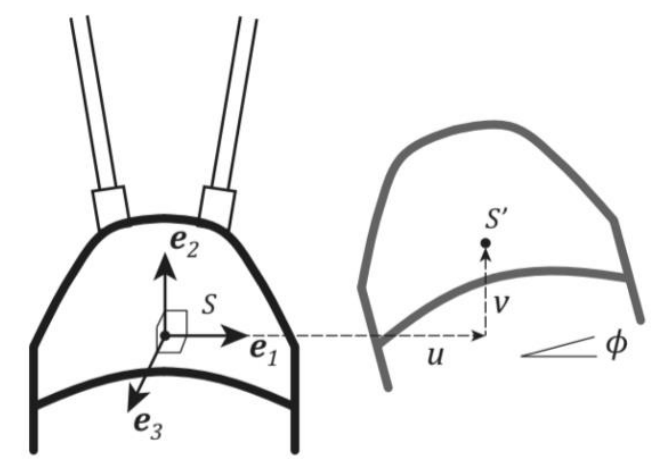

Figure 8 -Rim displacement [13]

Ford et al. [13] continued the development of the wheel model by studying its buckling dynamics in accordance to his new displacement definition. In his study, he finds that the rim's lateral stiffness controls the overall flexibility of the wheel model because it is much smaller than the radial stiffness and the lateral-torsional strength of the wheel dictates rim failure [13]. The spoke pretension plays a key role in this as it offers 2 purposes: helps to balance the spoke stiffness and controls the balance between wheel radial and lateral stiffness. The balance in spoke tension must ensures the spokes don't slack under load but also ensure that the ratio of radial strength to lateral strength is not too high which could result in high strain levels in the rim. Ford calculates a minimum tension so that the wheel can withstand the external loads to prevent any slackening, and a maximum tension limit, which if exceeded can lead to non-planar buckling or wheel collapse. The spoke tension is critical in the predicting the buckling limit of the wheel [13].

Petrone et al. [14] developed an experimental method for evaluating how the radial stiffness of high-performance racing wheels affects rider comfort. Various rim cross-sections, rim materials, spoke count, and spoke patterns were evaluated. The wheels underwent three different loading conditions using a servo-hydraulic cylinder MTS 242 including static press, cyclic press, and an impulse bump press (Figure 9). The radial stiffness was calculated for each of the wheel 
types in each of the load conditions. In the static press, a maximum load of $2000 \mathrm{~N}$ was reached to simulate a quasi-static radial overload on the wheel. In the cyclic test, a peak of $1000 \mathrm{~N}$ was applied at a $4 \mathrm{hz}$ frequency. This test simulated rolling on a flat surface at $30 \mathrm{kmh}$. The bump test introduced a $1500 \mathrm{~N}$ impulse load, with a load ramp rate of $22200 \mathrm{~N} / \mathrm{s}$. These tests evaluate the elastic response of the wheel under various loading conditions, but it did not replicate an impact condition that result in rim fracture.
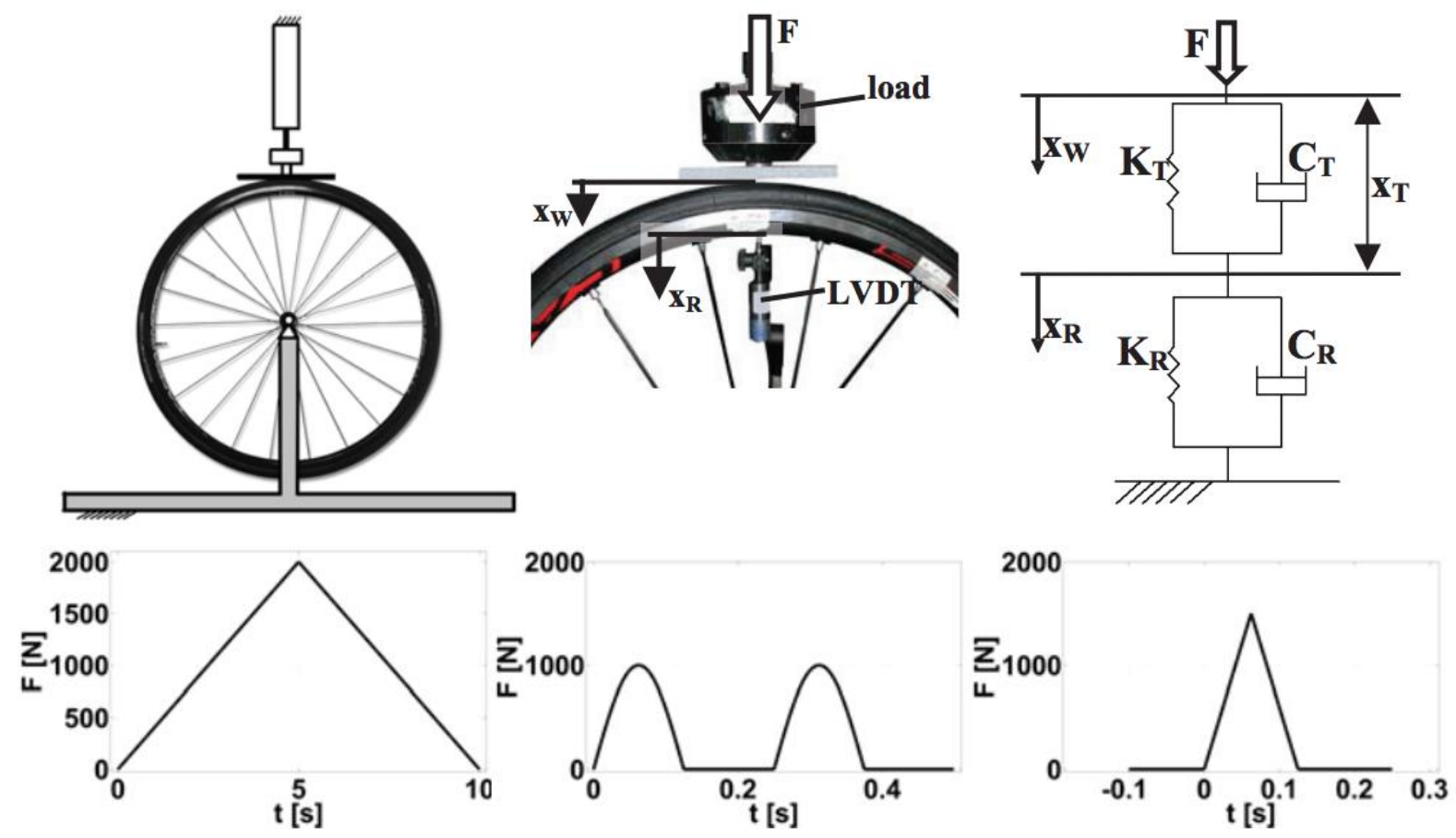

Figure 9 - Radial structure evaluation setup by Petrone et al. [14]

\subsection{Composite materials}

\subsubsection{Overview on composite materials}

A composite material can be defined as a material that is combination of at least two or more constituent materials or phases. The combination of these materials or phases promotes a significant change for the characteristics of the composite and thus allows material designers flexibility for the intended use case [15]. In general, the constituent materials consist of a reinforcement and a matrix. The reinforcement possesses load-carrying characteristics and is embedded into a matrix to improve its mechanical properties such as strength and stiffness [15]. This constituent could take the form of particulates and fibers. The matrix is used to bind the load 
carrying constituents together, enable load transfer and provide the structural stability so that the composite can be free standing [15].

Composite materials can be sub-divided into three common constituent pairings which include: (1) Polymer-matrix composite (PMC); (2) Metal-matrix composite (MMC); and (3) Ceramic-matrix composite. PMCs are composed of fibers such as carbon, graphite, and glass; and polymer-matrixes such as thermoplastics and thermosets. PMCs are relatively low in processing cost and weight, and polymers are the most common matrix used in industry. Polymers constitute of a long chain of reiterating units bonded together through a chemical reaction [15].

Thermoplastics such as polyethylene and Polyether ether ketone (PEEK) can be formed under heat and pressure, with an option of reversal. They provide high ductility, and high processing speed. Thermosets such as epoxy cannot be reversed under heat and pressure, however, they are preferred due to their high stiffness and structural rigidity. In the sporting industry, PMCs such as carbon fiber reinforced with epoxy resin matrix are predominantly used and is this case in this study.

Fibers affect the overall structural performance of the composite. Fibers are single filaments with a very small diameter. They can be aligned along one direction in either very long strands or random short strands. They absorb most of the applied load and therefore fiber selection is critical for the intended use case. Common fiber selection criteria include modulus, strength, thermal properties, thickness, tow size, ply flexibility, part curvature, sizing, cost and supply [16].

The combination of fiber and matrix into composite results in a material that blends the individual properties of each material [15]. For properties such as strength and stiffness, the volume fraction of the constituents is the indicator that determines how these properties can be calculated.

$$
\begin{aligned}
& E_{f 1}=E_{f} V_{f}+E_{m} V_{m} \\
& \sigma_{f 1}=\sigma_{f} V_{f}+\sigma_{m} V_{m}
\end{aligned}
$$

Carbon fiber reinforced polymers (CFRPs) when compared to metals have superior properties in specific strength and stiffness, durability, corrosion, resistance, fatigue and in design 
flexibility. Carbon fibers also do come with a few disadvantages. CFRP is brittle upon impact and susceptible to catastrophic failure. With minor failures, CFRP can also prove to be difficult to repair. CFRP is also expensive for raw materials and energy intensive in manufacturing. Carbon fibers are distributed in the forms of non-crimp fabrics and prepregs (pre-impregnated carbon fiber sheet with uncured epoxy).

Non crimp fabrics are used to bind unidirectional fiber layers to avoid misalignments. The benefits of overlaying straight tows by stitching is that the crimp may be avoided, resulting in consistency. The variables in the fabric include the number of layers in the fabric, and number of individual fibers in a single tow.

Pre-impregnated thermoset, also known as prepeg, is known to higher performance capabilities compared to fabrics but are also more expensive to produce. They are prepared as rolls or tapes, in which fabrics have the thermoset infused but not cured [15]. The infusion process creates a stability within the prepreg and allows the distribution of resin over the fibers to be more consistent than the resin transfer molding techniques used with dry fabrics. Prepregs require storage at low temperatures so resin does not melt and can be manually laid up or vacuum bagged for curing. They have finer control for volume fraction and are easier to laser-cut for complex molds.

\subsubsection{Impact on composite structures}

Impact events such as flying foreign objects, debris, and collisions are unavoidable in engineering applications. During manufacturing, it is also common for accidental tool drops to cause internal and barely visible damage in a composite. During an impact event, the impact energy can be absorbed by the composite until the damage mechanisms are activated which leads to failure modes such as delamination, matrix cracking, and fiber breakage [17]. This damage can significantly weaken the part [18].

A first step in understanding the impact tolerance of a part is to measure the damage imitation energy of a specimen [18]. By loading the specimen with progressively higher impact 
energies, the damage propagation can be observed. Many studies have been conducted with experimental and numerical solutions to describe impact tolerance and fracture propagation.

\subsubsection{Low velocity impact}

A well accepted definition of low velocity impact is an impact event such that the contact duration between the impactor and the surface is long enough for the composite structure to respond to the impact, thus more energy is absorbed elastically [18]. Liu et al. [17] furthered the definition to an event such that the main damage mode is delamination and matrix cracking. The difference in high velocity impacts is that, penetration or stress wave propagation can occur and the structure will not have enough time to respond resulting in a non-elastic behavior [19].

In low velocity impacts, damage usually initiates through matrix cracking. Interlaminar, tensile and shear strengths of the resin are the most influential parameters in determining impact tolerance, which is the energy level just before damage initiates [18]. The maximum stress criterion, which describes failure by comparing the tensile and shear strength to the current stress, is an analytical model used to determine when a crack can initiate [18]. If the crack is perpendicular to the orientation of the laminate, it is known as a tensile crack. If the cracks induce delamination between adjacent plies, it is due to a buildup of matrix cracking and high shear stresses [18].

While it is possible to predict an onset of damage, it is difficult to achieve a detailed description. Two approaches have been developed for damage prediction: (1) estimating the overall size of the area based on the impact point. Since the impact point will induce high stresses in that local area, impact defects will initiate [18]. The second approach (2) is to determine the impact energy level when the first appearance of matrix cracking. This can be done via experimental testing and finite element analysis [18].

\subsubsection{Composite Impact Parameters and Failure Modes}

Investigations over the past couple decades have studied parameters to determine their effects on the impact toughness of a specimen. Some of the most important include fiber orientation, interface strength, velocity of impact, drop weight, and specimen's dimensions [15]. 
Fiber orientations can influence composite failure threshold as it they can change which mode of failure is activated first. Figure 10 illustrates the out of plane energy threshold of a glass epoxy laminate, using unidirectional and cross-ply laminates. As the angle of the unidirectional and in-between the angle-ply changes, the activation threshold does as well. It can be observed from this study a 60-degree unidirectional laminate recorded the lowest impact energy threshold.

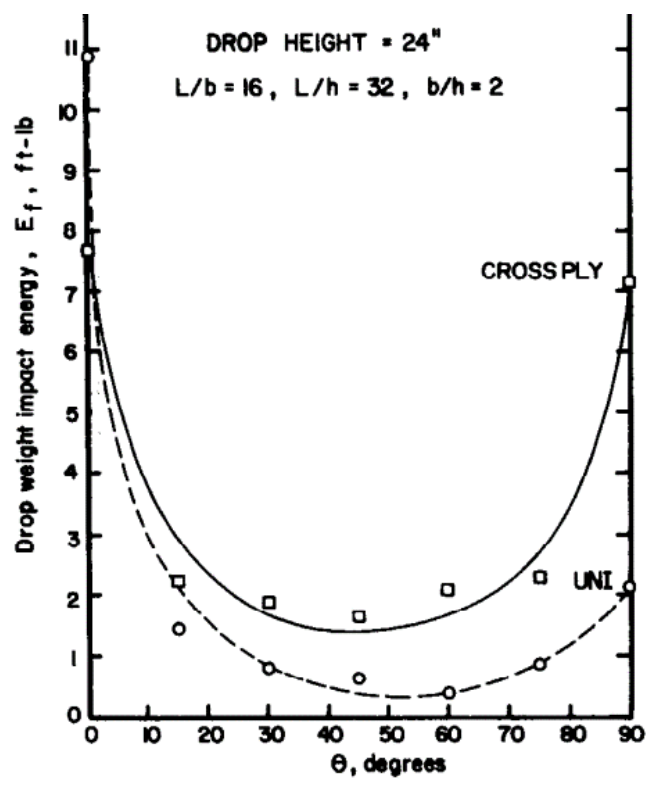

Figure 10 - Impact energy threshold vs ply angle [18]

Interface shear strength of the resin is also an important indicator in the overall impact strength of a composite. Shear strength can influence failure initiation, flexural strength, and resistance to interlaminar debonding [15].

There are 5 major impact low velocity damage failure modes in a composite material. They include delamination, fiber fracture, matrix fracture, fiber debonding and fiber pullout. The summation of the failure modes involved in the fracture process account for the total energy absorbed during impact. 


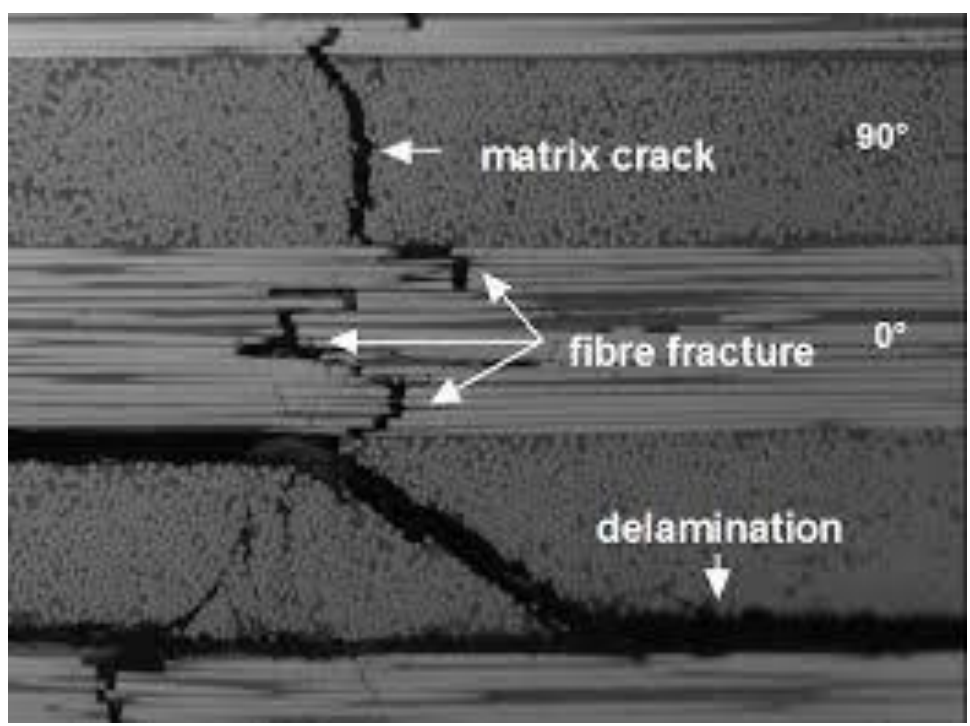

Figure 11 - SEM image of prominent CFRP failure mechanisms [20]

\section{Fibre breakage}

Fibre breakage (Figure 11) or fracture occurs when the fiber strain exceeds its maximum strain threshold. The crack that forms is perpendicular to the direction of which they align. Upon impact loading, fiber breakage usually occurs at the back face of the composite, where the face experiences its highest tensile stresses [15]. Generally, amongst the failure modes, fibre breakage accounts for a small amount portion of the total damage.

\section{Matrix Deformation and Cracking}

When a composite is exposed to an impact event, the matrix undergoes some deformation prior to fracture. When a crack propagates in one direction, the new surface produced is small resulting in a small amount of energy released (Figure 11) [15]. If cracks branch off in multiple directions, the sum of the energy absorbed from the secondary cracks is larger than the primary crack [15]. The secondary cracks usually happen when the stacking sequence is composed of cross and angle plies.

\section{Fiber Debonding}

In this failure mode, fibers separate from the matrix by cracks that run parallel to them. Chemical bonds between fibers and matrix are broken leading to a separation in the interface. This type of cracking occurs when there is a large discrepancy between fiber and matrix strength. If debonding is extensive, a significant amount of impact energy may have been absorbed [15]. 


\section{Fiber Pullout}

Fibre pullout occurs when brittle or discontinuous fibers are embedded in a tough matrix. Upon fracture, fibers which do not lie in the plane of fracture are exposed to a stress concentration in the matrix. This pressure is large enough to break the fiber at point and pull it before yielding the matrix [15]. The difference between fiber debonding and pullout is that fiber debonding takes place when a matrix crack is unable to propagate across a fiber, where as a fiber pullout is when the crack initiated at a fiber break is unable to propagate through a matrix leading to the fiber being pulled out at the crack [15].

\section{Delamination Cracks}

Delamination (figure 12) is the most common and most detrimental failure mode. Delamination occurs when a crack runs in between the interlaminar region of two adjacent laminas in the composite. As cracks propagate through a ply in a laminate, it may stop when it reaches the next ply. Due to high shear stresses in the matrix, the crack may branch off and start running at the interface parallel to the plane instead of through the plies [15]. As the crack grows, plies start to separate, and a delamination occurs. Delamination is responsible for absorbing the most amount of the fracture energy and occur frequently during impact. Composites with high shear strength can have better impact resistances [15].

\subsection{Impact testing}

\subsubsection{ASTM D7136 Low velocity impact testing for composite materials}

The most common method for testing low velocity impact of CFRP materials is the drop weight impact tester, defined by the ASTM D7136 standard. A typical drop weight tester consists of a drop tower with a rigid base, a drop weight impactor, a rebound catcher, and a guide mechanism [21]. In this method, a plate is placed in rigid supports and a known mass with a semi-spherical tip is dropped from a certain height guided by the rails. The catch mechanism ensures that multiple 
impacts do not occur. The mass and height of the falling weight are adjustable and are set to achieve desired impact velocity.

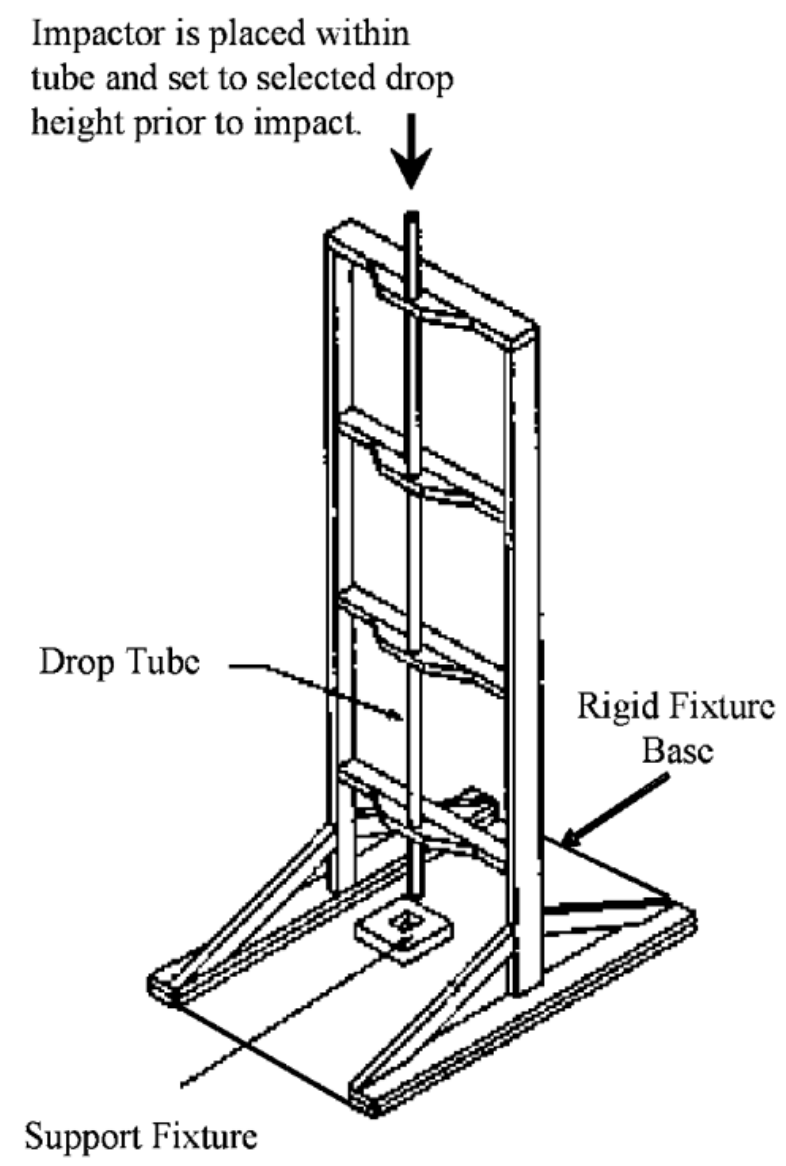

Figure 12 - Impact drop test ASTM D7136 [21]

The ASTM D7136 test is based off the conservation of energy principle in which load sensors and/or cameras can be used to monitor energy transfer. Although a high-speed camera, does not collect data on the impact force it does capture the impact and bounce velocities of the impactor. The difference in velocities equals the energy absorbed by the specimen.

$$
E_{\text {absorbed }}=\frac{1}{2} m\left(v_{o}^{2}-v_{f}^{2}\right)
$$

\subsubsection{UCI Impact test}

The UCI cycling body (Union Cycliste Internationale) has a wheel impact test for qualifying new wheel designs. In this test, a drop test impactor is required with a weight of 6-10 kg and dropped at a starting energy of $40 \mathrm{~J}$. The impactor shall have a flat steel anvil with an impact surface covered with a $20 \mathrm{~mm}$ thick rubber pad of hardness shore A50, and compression set 40\% [22]. 

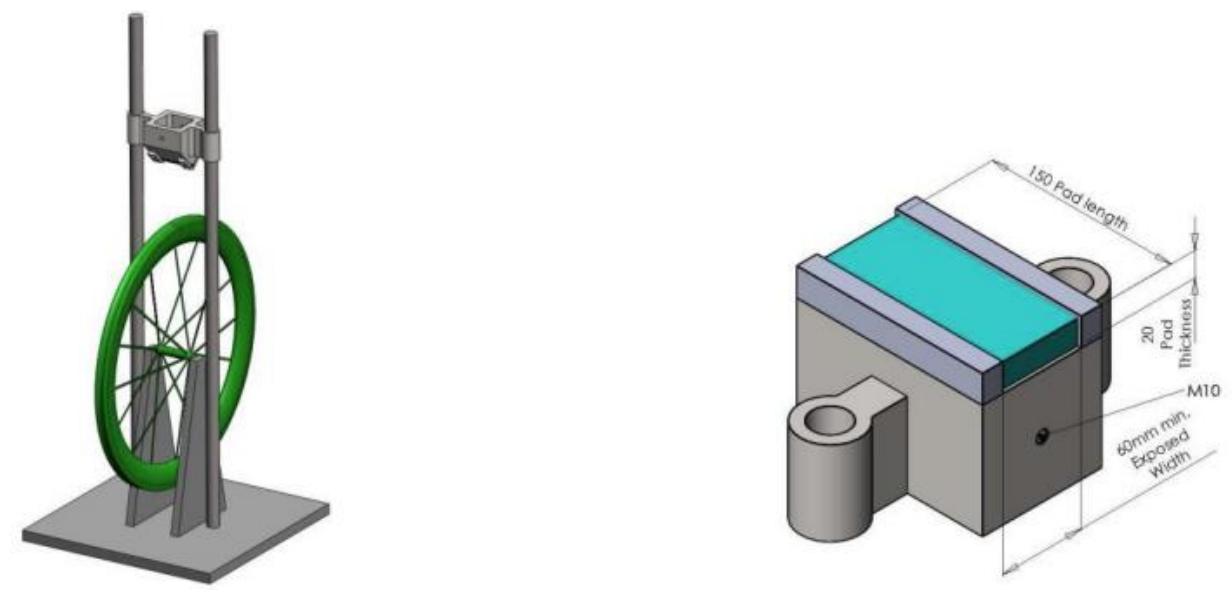

Figure 13 - UCI Wheel impact tester sample design [22]

The force compression of the A50 rubber matches that of a road tire with an average inflation pressure. The impact is released on a bare rim 90 degree from the valve hole. The failure criteria include: (1) No visible cracks or delamination (2) No change in lateral profile or lateral run in excess of $1.0 \mathrm{~mm}$ (3) No change in radial profile or radial run in excess of $1.0 \mathrm{~mm}$ [22]. The wheel design criteria for the test requires (1) rim height must be a minimum of $25.0 \mathrm{~mm}$ (2) Rim material can be alloy or composite (3) minimum of 20 spoke wheels (4) all components must be commercially available.

\subsection{IR and PCT}

\subsubsection{Section Infrared Thermography}

Infrared thermography (IRT) is the science of acquiring and processing thermal information from non-contact measuring devices [23]. Any object that is at a temperature above absolute zero, emits a form of electromagnetic radiation known as infrared radiation that has longer wavelengths than those of visible light [23]. Although the human eye is unable to pick up on this information, IRT can acquire this radiation. After capturing the radiation, a device can digitally process the data and create thermal images.

A thermal image, also known as a thermogram [24], is a visual representation of the heat emitted from objects in the frame. Each pixel is assigned a color in proportion to the range of infrared energies in the frame and thus objects can be easily differentiable. When there are inconsistencies across a surface or within a penetrable distance of the surface, an anomaly will 
appear. The anomaly can instigate further investigation which could lead to the discovery of defects. This method is very useful for monitoring damage across a number of applications [25].

IRT has many advantages over other measurement technologies such as X-ray, ultrasound, radiography, CT scan [25]. Firstly, IRT is non-contact. It can objectively measure the temperature of objects without getting into contact with extremely hot, corrosive or acidic products. IRT is noninvasive and can monitor from a distance and provides two-dimensional thermograms which allow you to compare visual and thermal data. IRT can be measured in real time and with high speed imaging therefore suitable for impact applications [25]. IRT is not limited by lighting conditions [23] and there are no harmful radiation effects such as those from X-ray. It is therefore capable of prolonged usage.

Infrared thermography also comes with a couple notable disadvantages including limited information on defect depth and other quantitative characterizations of defects. Recent studies have been actively involved in improving and progressing the quality of the infrared thermography data through better signal to noise ratios [25] and post-processing methods. Developments have also been made to advance thermal diffusivity estimation which can help to reduce the error where reflected heat can overshadow the emitted heat resulting in inflated readings, as is the case with metals [25].

Infrared thermography has been implemented with two different approaches. The first is known as passive infrared detection. This is when radiation is measured without any external heat excitation. In this situation, temperature and process behavior can be monitored and any inconsistent divergence of temperature can signal physical changes. In addition, impact situations can be monitored as kinetic energy passes through the impactor to the specimen and if there is a permanent deformation, energy is dissipated as heat across the surface of the specimen [26]. Passive monitoring is very useful in quality control and process monitoring applications [23].

The second approach is known as active infrared detection and is most commonly used in nondestructive testing of applications. In this approach, an external stimulus is applied to the specimen to increase thermal contrasts on surfaces or regions of interest. Active thermography can be used 
to inspect materials for surface properties and defects. However, if anomalies are very subtle they can be lost in thermographic data noise [27]; which has facilitated the development of postprocessing techniques to help filter unwanted signals.

The most popular excitation techniques used in infrared thermography include the application of flash or halogen lamps [25]. These sources emit pulses of energy which can be either modulated or continuous. These options allows the inspection process to control the amount of heat, temperature ramp rate, and exposure time to create the optimal contrast for the receiving materials.

\subsubsection{Impact response using infrared thermography}

For evaluating impact response of material specimens, infrared thermography has been utilized as an effective tool as it can monitor surface temperature variations [23]. It has been shown that there are three different response behaviors during impact: thermoelastic effects which mean no damage has occurred, small temperature increase accounting for light delamination and micro cracks, and thermoplastic effects which can be illustrated by strong temperature rises [23].

During an impact event when damage is initiated, the dissipation of heat is non-inform. The distribution of heat manifests itself through the appearance of hot spots/areas over the surface, and this characteristic can be picked up by the thermal imaging device [28]. By evaluating temperature before and after an impact event, the damage progression can be analyzed [28].

\subsubsection{Active Non-Destructive Testing}

Active non-destructive testing has been used in various applications including inspection of aircrafts, energy systems, and medical imaging. There are two considerations to ensure that the NDE can be successful: (1) that structural continuity invariably produces good thermal continuity and (2) manufacturing defects such as voids, delamination, and foreign matter affect the flow of thermal energy across the laminar layers [25].

To perform a non-destructive evaluation a few key pieces of equipment are needed. The first is a heat source which controls the flow of thermal energy towards a specimen under inspection. The 
IR camera which provides live monitoring of the surface of the specimen under test. And a data acquisition system to store the data and allow processing.

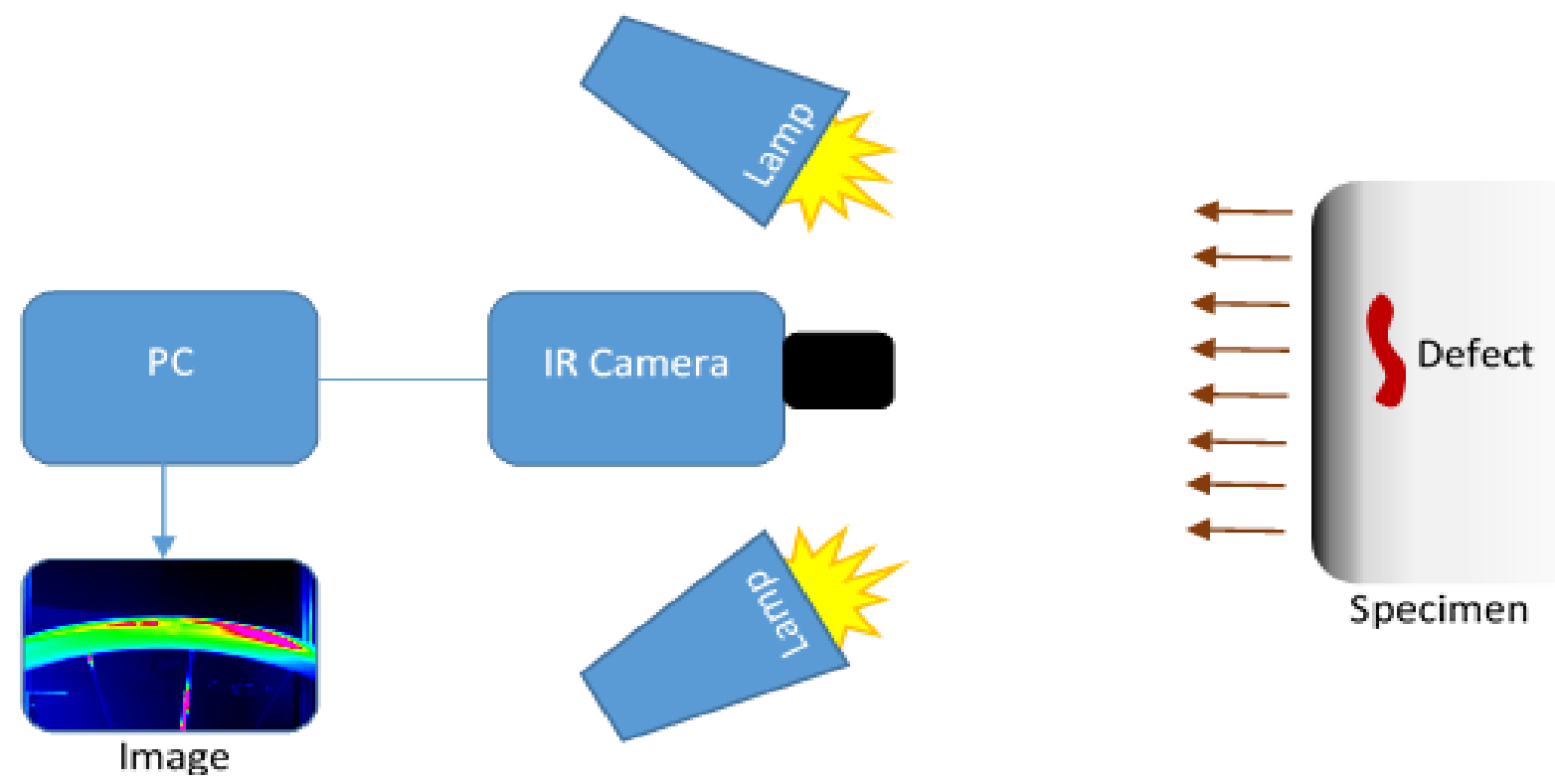

Figure 14 - IR thermography setup

The first phase in the evaluation is the initial heating. Heat can be applied continuously or in pulses, and the length of time is dependent on the material being studied. When heat is applied to a composite material, heat propagates through the surface and into the defected regions. As the heat source is removed, the surface cools and the thermal flow shapes out the discontinuities through to a difference in the heat release rate. The discontinues show up in voids, delamination's or unbonded regions. To perform more detailed analysis such as calculating defect depth, or provide better contrast, post filtering processing techniques such as principal component thermography, pulsed phase thermography, and polynomial fit can be performed [23].

\subsubsection{Post Processing - Principle Component Thermography}

Principal component analysis, or PCA, is a mathematical technique that synthesizes data sets to reduce the number of variables while losing the least amount of information. It also helps in highlighting the differences and similarities within the variables (1). In principal component thermography, this approach reduces a matrix of observations to its spatial and temporal variations. When plotted, the image can relate temporal variations to flaws in the structure [29]. 
To setup PCA for thermal inspection, a thermal video sequence must be condensed into a 2D matrix form with dimension $M x N$. The thermal inspection video can be defined as a threedimensional array, $T(i, j, k)$ where $i=$ pixel values in horizontal $(N x), \mathrm{j}=$ pixel values in the vertical $(N y), k=$ time $(n t)$. In order to condense the video, the first step is to concatenate each frame, which is a 2-dimensional array of pixels (256 x 320) into a long column (81,920 x 1). After the first column, the values in each successive pixel column are placed under the column preceding it. The A matrix is then comprised of each column representing a frame in the time series. Where $M=N x \times N y$ and $N=n t$.

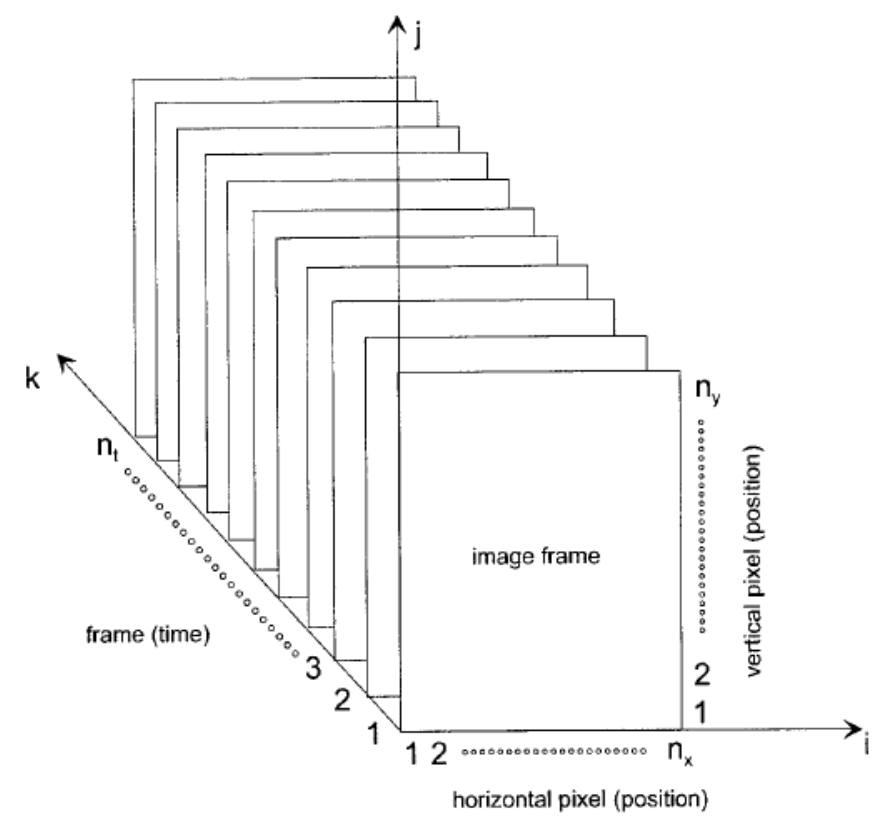

$$
A=M x N=(N x \times N y) \times N t
$$

Figure 15 - Sequence of $\mathrm{Nt}$ image frames each with $\mathrm{NxNy}$ elements for $\tilde{\mathrm{A}}$ matrix in principal component thermography [30]

Each $M$ column vector, which contains the pixel from a single frame, is then subjected to a $z$-score standardization process [30].

$$
\begin{gathered}
\tilde{A}(n, m)=\frac{A(n, m)-\mu_{n}}{\sigma_{n}} \text { where } \\
\mu_{n}=\frac{1}{N_{t}} \sum_{n=1}^{N t} A(n, m) \text { and } \\
\sigma_{m}^{2}=\frac{1}{N_{t}-1} \sum_{n=1}^{N_{-} t}\left(A(n, m)-\mu_{n}\right)^{2}
\end{gathered}
$$


Using the singular value decomposition method, the standardized matrix $\tilde{\mathrm{A}}$ is then reduced as per $A=U \Gamma V^{t}$ where, if $M>N, \Gamma=N \times N$ diagonal matrix with positive or zero elements representing singular values of A. $U=M \times N$ matrix and $V^{t}=(N \times N)^{t}$. The columns of matrix U contain the orthogonal modes which describe the spatial and temporal variations [30]. By reversing mode 1 , which is the second column of $U$, into a matrix of the original $N_{x} x N_{y}$ frame size, the principal mode can be plotted and displayed [28]. The efficiency of this method stems from replotting of information according to its largest temporal scales which is in the primary mode [23]. The principle modes eliminate noise and non-uniform temperature gradients due to environment. This technique also proves to be a very useful tool for reduction in data size without loss of important information [30]. Cramer et al. [31] outlines the steps to perform PCT in more detail, and Iberra-Castenado et al. [32] compares the effectiveness of this method with other postprocessing techniques. 


\section{Chapter 3}

\subsection{Bicycle wheel and material characterization}

This section is split up into 2 parts. In the first section, the design, composite properties, and manufacturing process of the bicycle wheel will be evaluated. By identifying weaknesses in these processes, improvements for increasing impact tolerance can be determined. In the second part of this section, the design of the impact apparatus and procedure will be discussed.

\subsection{Wheel design parameters and layup}

The wheel that is being used in this test is a tubular racing wheel. A tubular rim differs from a classic bicycle clincher rim, in which the top of the rim is enclosed whereas the clincher rim has overhangs that constrain the tire from getting loose. The tubular tire and casing are sewn together and glued onto the surface of the rim. The rim has a semi-deep cross-section which provides performance advantages such as increased aerodynamic efficiency, and a greater moment of inertia which adds structural rigidity [5]. The deeper cross section also offsets the need of additional spokes, helping to reduce weight [5]. Figure 16 highlights the physical specifications of the wheel. Table 1 lists the physical specifications of the wheel.
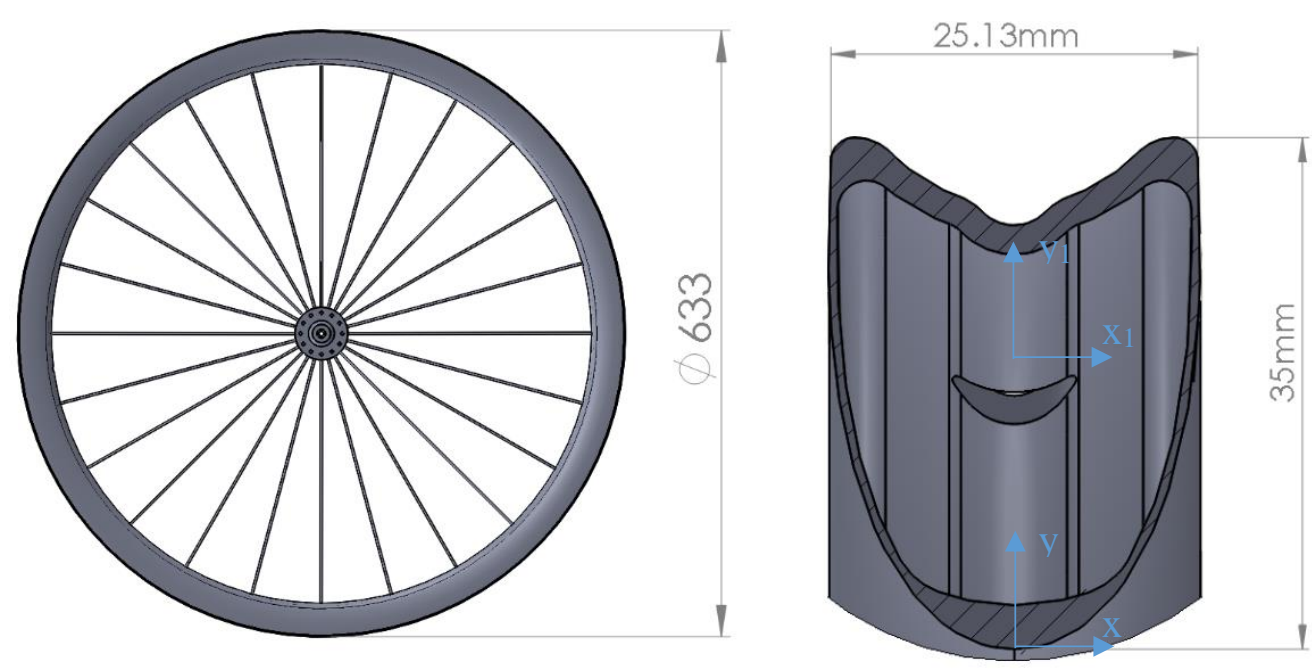

Figure 16 - Size and cross section of wheel under test 
Table 1 - Physical specifications of wheel under test

\begin{tabular}{|c|c|c|c|}
\hline Specification & Value & Specification & Value \\
\hline Weight & $445 \mathrm{~g}$ & Moment of Inertia $\left(\mathrm{I}_{\mathrm{xx}}\right)$ & $8445.8 \mathrm{~mm}^{4}$ \\
\hline External/Internal Width & $25.13 / 17 \mathrm{~mm}$ & Moment of Inertia $\left(\mathrm{I}_{\mathrm{yy}}\right)$ & $21339.2 \mathrm{~mm}^{4}$ \\
\hline Cross-sectional Area & $131.2 \mathrm{~mm}^{2}$ & Polar Moment of Inertia & $29785 \mathrm{~mm}^{4}$ \\
\hline Effective rim diameter (ERD) & $586 \mathrm{~mm}$ & Centroid & $(0 \mathrm{~mm}, 20.3 \mathrm{~mm})$ \\
\hline
\end{tabular}

Torayca 700 (T700) prepeg has been used as the production material for this wheel. This prepeg is widely utilized across aerospace, automotive, and sporting industries and has a great reputation for processability [33]. The T700 has a good balance of strength, brittleness, density, and price compared to its alternatives T300 and T800. The fiber volume fraction is 55\%. Table 2 lists the material properties of a cured prepreg lamina.

Table 2 - Mechanical properties of T700 2510 prepeg [33]

\begin{tabular}{|c|c|c|c|}
\hline Property & T700(Gpa) & Property & T700 (Gpa) \\
\hline$E_{1 \mathrm{t}}$ & 128 & $\sigma_{2 \mathrm{c}}$ & 0.283 \\
\hline $\mathrm{E}_{2 \mathrm{t}}$ & 9 & $\sigma_{1 \mathrm{c}}$ & 1.400 \\
\hline $\mathrm{E}_{1 \mathrm{c}}$ & 114 & $\mathrm{G}_{12}$ & 5.22 \\
\hline $\mathrm{E}_{2 \mathrm{c}}$ & 14.1 & $\tau_{12}$ & 0.159 \\
\hline$\sigma_{1 \mathrm{t}}$ & 1.682 & $\varepsilon_{\mathrm{t}}$ & $1.7 \%$ \\
\hline$\sigma_{2 \mathrm{t}}$ & 0.053 & $v_{\mathrm{xy}}$ & 0.35 (unitless) \\
\hline
\end{tabular}

The wheel has been split into 17 sections (Figure 17) of which there are 8 left-right mirrored pairs and a top middle section. The ply layup and stacking sequence of the wheel was provided by the manufacturer (Table 4). Key features include the use of ply tapering which allowed weight savings in the vertical sections of the rim. There is also a taper in the top section between the outer TL1/TR1 and inner TL/TR pair, and in R5/L5 near the bottom where the spoke hole is located.

The thick layer at the bottom of the cross section has been added to provide the strength to hold a pre-tensioned spoke (L5/R5), and the additional plies on top of the wheel (TL/TR) can support the tire pressure and systematic loads. In total, there are 40 plies used to layup the composite for this design. However, 2 plies are used for each layer, making 20 ply layers (Table 3). They are numbered in the order of which they are installed in the mold. The orientation of the plies is with respect to a cylindrical system in which the 0 degree is aligned tangentially to the wheel (i.e. along the circumference), 90 degree to be radially aligned, and the 45-degree plies to be forward facing when rolling. 

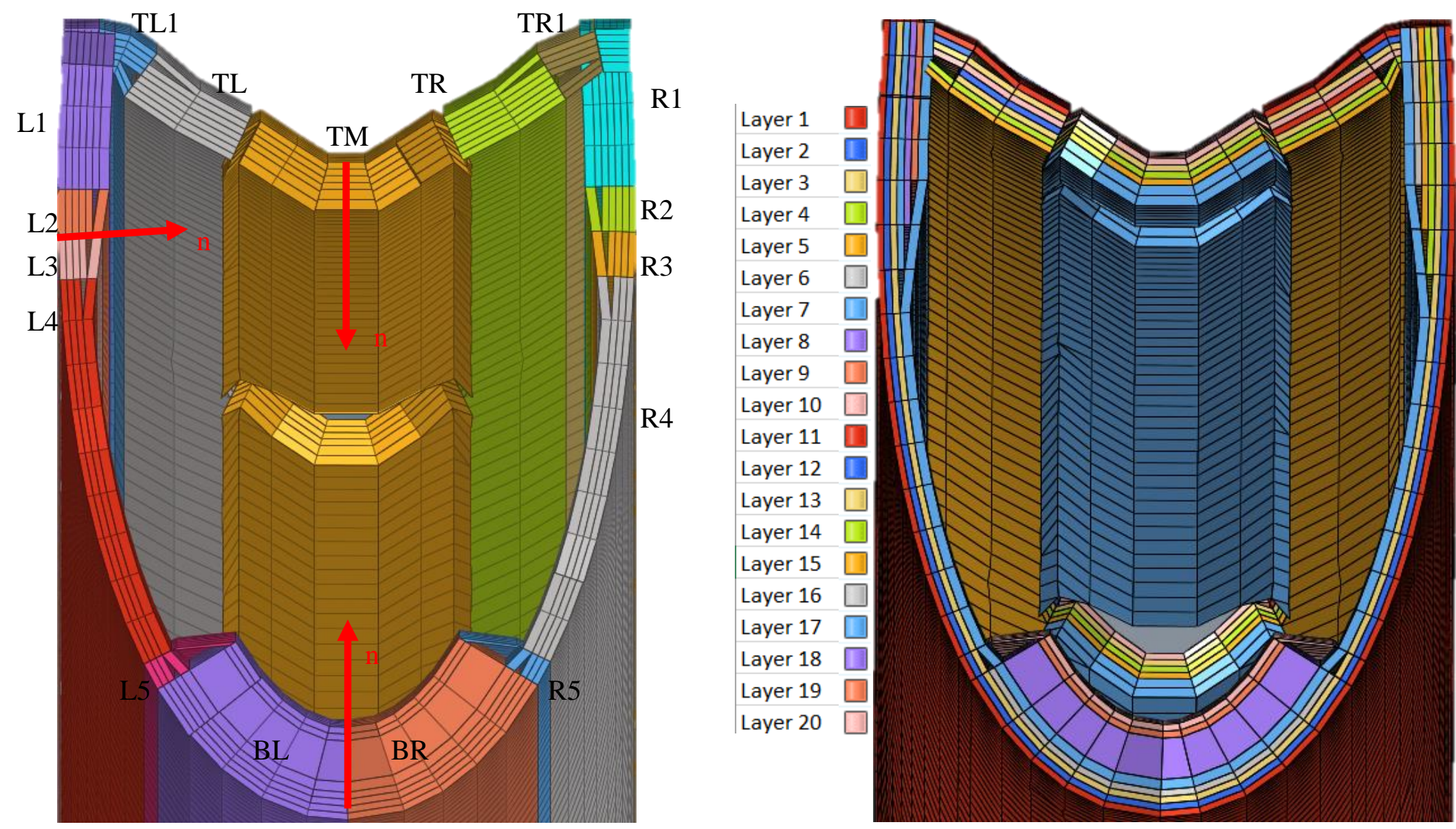

Figure 17 - Zone based and layer-based model of the wheel layup and stacking sequence 
Table 3 - Ply characteristics

\begin{tabular}{|cccc|cccc|}
\hline Layer & $\boldsymbol{\theta}$ & $\mathbf{T}(\mathbf{m m})$ & Region & Layer & $\boldsymbol{\theta}$ & $\mathbf{T}(\mathbf{m m})$ & Region \\
\hline 1 & $45_{2}$ & 0.3 & $\mathrm{TL} \rightarrow \mathrm{TR}($ sides $)$ & 11 & $90_{2}$ & 0.3 & TL \\
\hline 2 & $90_{2}$ & 0.3 & $\mathrm{~L} 1 \rightarrow \mathrm{R} 1($ sides$)$ & 12 & $90_{2}$ & 0.3 & TR \\
\hline 3 & $45_{2}$ & 0.3 & $\mathrm{TL} \rightarrow \mathrm{TR}($ sides $)$ & 13 & $90_{2}$ & 0.3 & TL, TM, TR \\
\hline 4 & $90_{2}$ & 0.3 & $\mathrm{~L} 1 \rightarrow \mathrm{L} 3$ & 14 & $90_{2}$ & 0.3 & TL1 $\rightarrow$ TR1 (along top) \\
\hline 5 & $90_{2}$ & 0.3 & $\mathrm{~L} 1 \rightarrow \mathrm{L} 2$ & 15 & $90_{2}$ & 0.3 & TL1 $\rightarrow$ TR1 (along top) \\
\hline 6 & $90_{2}$ & 0.3 & $\mathrm{~L} 1$ & 16 & $45_{2}$ & 0.3 & BL, BR \\
\hline 7 & $90_{2}$ & 0.3 & $\mathrm{R} 1 \rightarrow \mathrm{R} 3$ & 17 & $0_{2}$ & 0.45 & $\mathrm{~L} 1 \rightarrow \mathrm{R} 1, \mathrm{TM}$ \\
\hline 8 & $90_{2}$ & 0.3 & $\mathrm{R} 1 \rightarrow \mathrm{R} 2$ & 18 & & 1.8 & $\mathrm{BL}, \mathrm{BR}-\left[0_{2} / 45_{2} / 0_{2}\right]_{\mathrm{s}}$ \\
\hline 9 & $90_{2}$ & 0.3 & $\mathrm{R} 1$ & 19 & $0_{2}$ & 0.45 & $\mathrm{TM}$ \\
\hline 10 & $90_{2}$ & 0.3 & $\mathrm{TL} 1 \rightarrow \mathrm{TR} 1$ (top) & 20 & $45_{2}$ & 0.3 & $\mathrm{~L} 5, \mathrm{BL}, \mathrm{BR}, \mathrm{R} 5$ \\
\hline
\end{tabular}

Layer 1 is the outermost layer and installed first in the mold. It encompasses the outside perimeter including the left and right walls and folds over TL and TR of the top section. Layer 2 is similar to layer 1, as it covers the walls and the bottom, but does not fold onto the top section. Layer 3 is the same as layer 1 . Layer $4 \rightarrow 6$ run down the vertical left wall of the wheel. Layer 7 $\rightarrow 9$ is the mirror copy of layer $4 \rightarrow 6$ on the right side. Note: In table 4 , the layer column is notated with the left sided identifiers, but the stacking sequence are the same on the right side.

The bottom plies are then installed with layer 16 covering BR and BL. Layer 17 wraps all the plies from the inside of the cross section, and after the bladder mold is installed, folds over and supports the top section. Layer 18 and 19 are then added on top of layer 16 to establish the base for the spoke nipples. Layer 20 covers the bottom and ends at L5/R5. An internal bladder mold is then installed in the core of the wheel to compact the bottom plies, and as layer 17 is wrapped over the bladder a double fold is created. The top of the structure is then completed with the layering of plies in reverse order, 15 to 10. 
Table 4 - Homogenized membrane properties of zone-based wheel sections

\begin{tabular}{|l|l|l|c|c|c|c|c|}
\hline \multicolumn{1}{|c|}{ Zone } & $\begin{array}{c}\text { Layers } \\
\text { (n= normal } \\
\text { (Figure 17, bottom } \\
\text { up stacking) }\end{array}$ & \multicolumn{1}{|c|}{ Layup } & $\begin{array}{c}\boldsymbol{t} \\
\mathbf{m m}\end{array}$ & $\begin{array}{c}\mathbf{E}_{\mathbf{1 1}} \\
\mathbf{G p a}\end{array}$ & $\begin{array}{c}\mathbf{E}_{\mathbf{2 2}} \\
\mathbf{G p a}\end{array}$ & $\begin{array}{c}\mathbf{G}_{\mathbf{1 2}} \\
\mathbf{G p a}\end{array}$ & $\mathbf{v}_{\mathbf{1 2}}$ \\
\hline $\mathrm{TM}$ & $10 / 13 / 14 / 15 / 17 / 19$ & {$\left[90_{8} / 0_{4}\right]$} & 1.8 & 17.1 & 58.9 & 5.2 & -0.03 \\
\hline TR/TL & $1 / 3 / 10 / 11 / 13 / 14 / 15$ & {$\left[45_{4} / 90_{10}\right]$} & 2.1 & 11.1 & 73.8 & 6.6 & -0.05 \\
\hline TR1/TL1 & $1 / 2 / 3 / 10 / 14 / 15$ & {$\left[\left(45_{2} / 90_{2}\right)_{2} / 90_{4}\right]$} & 1.8 & 11.6 & 77.5 & 7.9 & -0.06 \\
\hline R1/L1 & $1 / 2 / 3 / 4 / 5 / 6 / 17$ & {$\left[\left(45_{2} / 90_{2}\right)_{2} / 90_{4} / 0_{2}\right]$} & 2.25 & 20.4 & 74.3 & 7.2 & -0.07 \\
\hline R2/L2 & $1 / 2 / 3 / 4 / 5 / 17$ & {$\left[\left(45_{2} / 90_{2}\right)_{2} / 90_{2} / 0_{2}\right]$} & 1.95 & 19.8 & 65.8 & 7.7 & -0.07 \\
\hline R3/L3 & $1 / 2 / 3 / 4 / 17$ & {$\left[\left(45_{2} / 90_{2}\right)_{2} / 0_{2}\right]$} & 1.65 & 20.3 & 53.1 & 8.5 & -0.07 \\
\hline R4/L4 & $1 / 2 / 3 / 17$ & {$\left[45_{2} / 90_{2} / 45_{2} / 0_{2}\right]$} & 1.35 & 23 & 27.6 & 9.6 & -0.1 \\
\hline R5/L5 & $1 / 2 / 3 / 17 / 20$ & {$\left[45_{2} / 90_{2} / 45_{2} / 0_{2} / 45_{2}\right]$} & 1.65 & 38 & 24.2 & 11 & -0.16 \\
\hline BR/BL & $1 / 2 / 3 / 17 / 18 / 19 / 20$ & {$\left[45_{2} / 90_{2} / 45_{2} / 0_{2} /\left(0_{2} / 45_{2} / 0_{2}\right)_{s} / 45_{2}\right]$} & 3.7 & 87.3 & 16.3 & 9.9 & -0.38 \\
\hline
\end{tabular}

For each section, the homogenized membrane stiffness values were calculated. $\mathrm{E}_{11}$ corresponding to the modulus of the laminate around the central axis of the wheel (along the circumference of the wheel). For the vertically oriented sections (R1/L1 $\rightarrow$ R5/L5), E 22 corresponds to the modulus of laminate in line with the radial axis of the wheel (running radially from the center of the wheel). For the laterally oriented sections (TL1 $\rightarrow$ TR1 \& BL/BR), $\mathrm{E}_{22}$ corresponds to the modulus of the laminate in the lateral direction (out of plane).

From the modulus values, each section contributes uniquely to the structural performance of the wheel. The top sections provide lateral stiffness to the wheel as they withstand the constant pressure from the tire. The shear stiffness is especially low in the top middle section. At the rim sidewalls, $\mathrm{E}_{22}$ - the radial stiffness, is high as expected as it transfers load from the top section into the bottom section. The effects of ply tapering can be seen throughout the sidewalls as $\mathrm{E}_{22}$ is decreasing from $\mathrm{R} 1$ to $\mathrm{R} 4(\mathrm{R} 1>\mathrm{R} 2>\mathrm{R} 3>\mathrm{R} 4)$ ). In the last $\mathrm{R} 5$ section is slightly strengthened from R4 with the addition of layer 20, however a large resin area that is unaccounted for in this calculation makes it more susceptible to delamination and ply separation. The bottom zones are highly strengthened with the extra layers that give it good longitudinal and shear strength. The bottom of the wheel must resist torsion from braking and hold the spoke tension. It can be noted, that none of the layup zones are symmetric and balanced. This can induce unwanted shear strains, normal stresses and bending moments during the curing process which can lower the wheels resistance to additional loads [34] [35]. 


\subsection{Manufacturing overview}

\subsubsection{Internal bladder molding}

To manufacture the wheel, the prepeg must consolidate to the walls of a mold while having a dimensionally consistent hollow core. A good method to achieve this is by using the internal bladder molding technique. This technique allows a consistent pressure to be applied from the inside surface and once removed, creates the hollow channel throughout the wheel [36]. In addition to consolidation, internal bladder molding also assists the prepegs in good dimensional consistency [37]. Internal bladder molding is limited in precision due to the placing of the bladder being a manual process, the flexibility of the bladder material can cause warpage, and low drapability in small radii [36]. If the bladder becomes loose or is not sized within tolerance, it can rattle and create sink marks [38].

There are various methods to produce the internal bladder. In this application a highdensity polyurethane foam core is used. This material resists the resin which prevents soaking and reduces the chances of resin rich or starved areas. The foam is machined to the geometric tolerances of the hollow core. Before applying, the prepregs are first cut to the desired shape, and placed in the bottom half of the mold. The foam core is then added, followed up with the layup of the top half. The mold can be sealed, pressurized, and then heated to accelerate the curing process. The core can then be removed in an acid or water bath, where the polyurethane dissolves and is washed out.

Although internal bladder molding is a great technique for the manufacturing of highperformance bicycle wheels, it is still susceptible to defects. The defects can arise due to design, material, and manufacturing control issues [39]. The most common defects such as voids and porosity are caused from air pockets, moisture or lack of vacuum control inside the mold [36]. Delamination is due to moisture, lack of pressure, and incorrect thermal settings. Wavy Fibers occur when there is a lack of dimensional control, prepegs or internal bladder is not cut to the right size, and a presence of free space [38]. Resin-rich or resin starved areas due to processing issues of the prepegs. Appearance of foreign objects can occur from an unclean work environment resulting in a contamination of the cured part. 


\subsubsection{Manufacturing quality review}

An optical inspection was performed to identify manufacturing defects that could deter the impact performance of the wheel. Currently these wheel models must pass an acoustic tap test to check for manufacturing flaws before they are accepted for release. The tap tests are subjectively passed without any vibration measuring devices, and often do not capture performance hindering defects. Once the wheels are released there is no way to track inherited deficiencies, thus it is necessary to evaluate. A summary of the most common defects seen across 10 sample cross sections are highlighted below.

To prepare a sample, a cross-section was cut off the wheel with diamond table saw cutter and set in LECOSET 7008 cold curing resin. The puck was polished until a final grit of 5 microns. Sections which line up with the center of the spoke hole are included in the discussion as they show more prominent defects. The drilling process for the spoke holes induces micro failures which can be seen at the edges. These did not exist in the sections without the spoke holes.

In Figure 18a, the cross section of the wheel is shown. Figure 18b shows a separation between the bottom most layer and the rest of the top section. This problem raises concerns over the fit of the internal bladder mold and the curing settings of the manufacturing process. Figure $18 \mathrm{c}$ highlights the edge of the spoke installation hole. Holes are stress releases and can initiate cracks. The addition of layer 11 can also been seen with an acceptable resin pocket. In Figure 18d, the top right corner shows larger degree of layer separation and resin rich areas. The layer also seems to have shifted from its intended location, TR, into TR1. Figures 18e,f,j and $\mathrm{k}$ show the ply tapering features of sections L2, L3 and R5, R4 respectively. Figures 18g and 18h, show the top and bottom sections of the spoke nipple mount area on the rim cross section. The edges here are rough, and some ply separation can be seen. This is another area susceptible for crack initiation. Figure 18i shows the junction where the weave and layer 19/20 are introduced. 

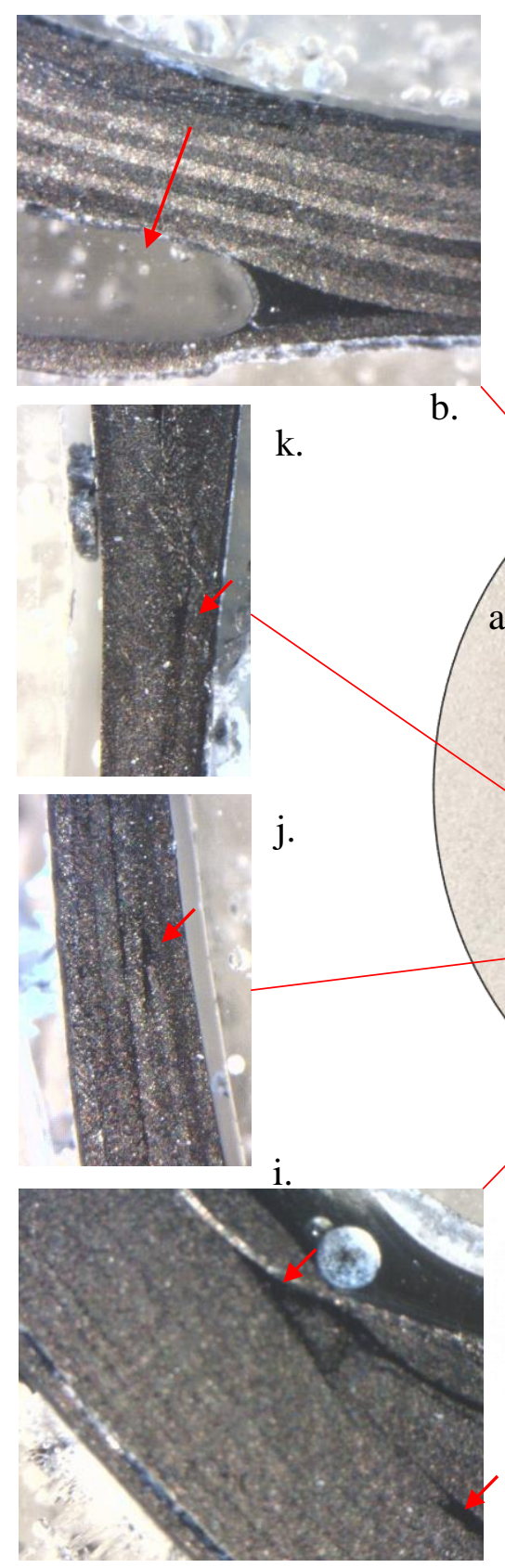

b.

$\mathrm{k}$.
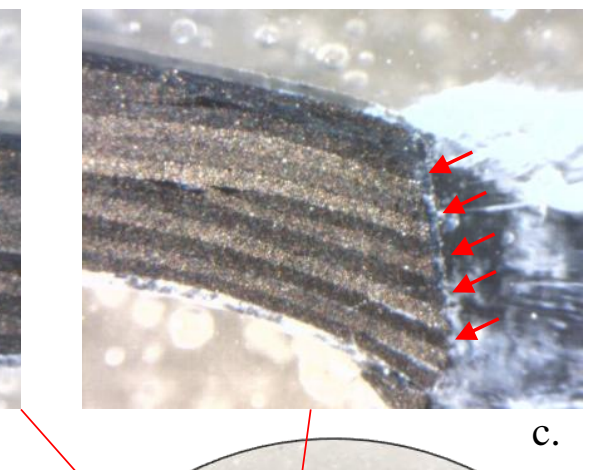

d.
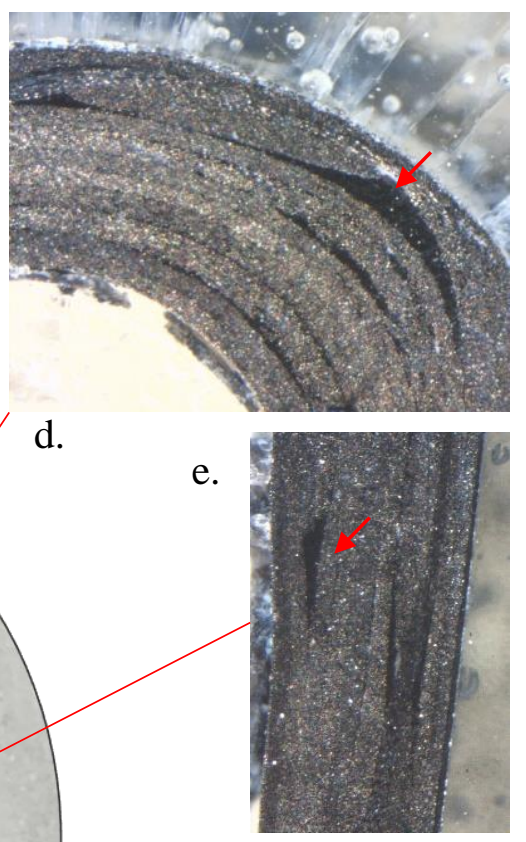

.

Figure 18 - Optical inspection of manufactured wheel cross section - (a) wheel cross section (b) top delamination (c) top wavy fibers and hole edge (d) wavy fibers and resin rich areas at top left corner (e) ply tapering and resin rich area - R2 (f) ply tapering and resin rich area $-\mathrm{R3}$ (g) top of right bottom hole edge with micro cracks (h) bottom of left bottom hole edge with resin rich areas and delamination (i) delamination at L5 (j) new ply introduction at $\mathrm{L5}(\mathrm{k})$ ply tapering and resin rich area $-\mathbf{R 4}$ 


\subsection{Experimental Setup}

\subsubsection{Design of Impact Test Apparatus}

Although there is no standard protocol for performing a low velocity impact test on a bicycle wheel, the UCI impact test and the ASTM-D7136 can be used as a reference for the design of the apparatus. Since, the university does not have access to one, an impact test machine was designed and manufactured to perform the low velocity impacts and with the capabilities of impacting the wheels to failure. This apparatus allows one to evaluate how much impact energy needs to be introduced to activate the damage mechanisms of the carbon fiber rim. The design of the apparatus is shown in Figure 19.

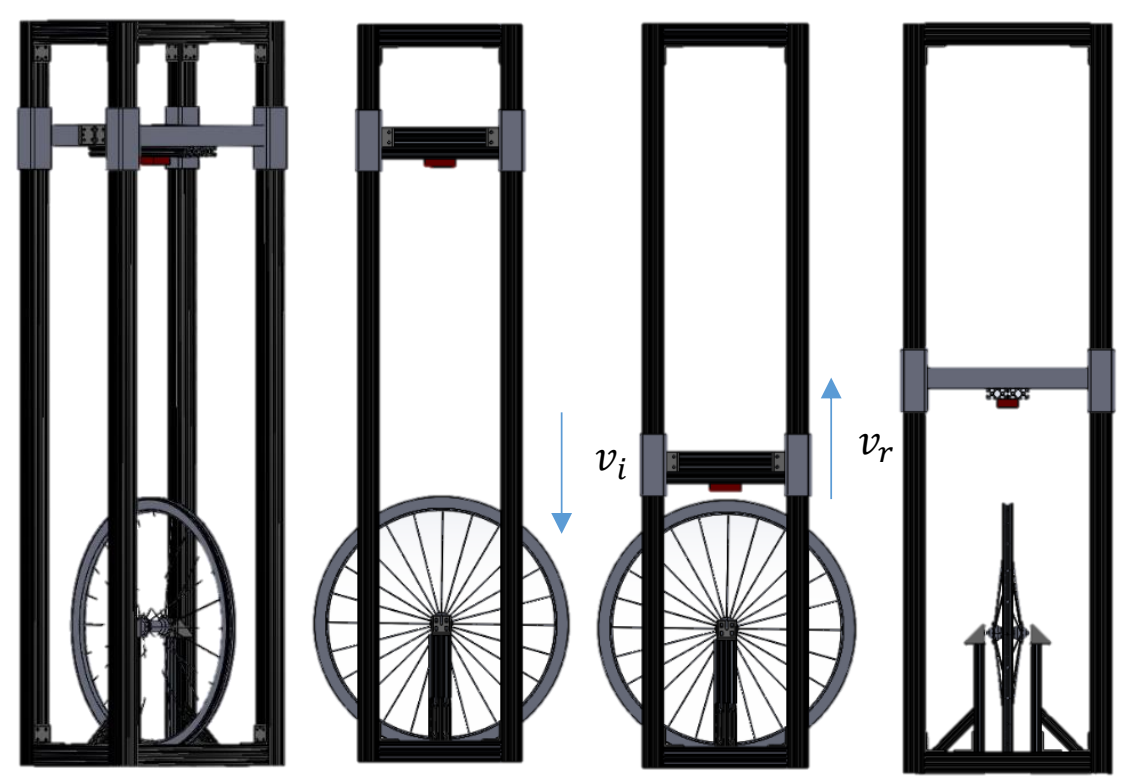

Figure 19 - Impact test design

This test works on the energy conservation principle, in which a mass (m) is dropped from an initial height $\left(h_{i}\right)$ to impact the specimen. The impact energy $\left(E_{\text {impact }}\right)$ can be calculated by $h_{i}$ and the gravitational $(\mathrm{g})$ potential energy of the mass at this height. The rebound energy $\left(E_{\text {rebound }}\right)$ is the gravitation potential energy of the mass at the rebound height. The difference in the initial height ( $h_{i}$ - height pre-set before impact) and the rebound height $\left(h_{r}\right.$ - height of the weight post impact) is directly proportional the energy absorbed by the specimen $\left(E_{\text {absorbed }}\right)$. The impact velocity, $v_{i}$, is the velocity of the mass just before striking the wheel and the rebound velocity, $v_{r}$, is the velocity of the mass just as it bounces off the rim. The equations $7-9$ highlight this principle [21]: 


$$
\begin{gathered}
E_{\text {absorbed }}=m g h_{i}-m g h_{r} \\
E_{\text {impact }}=m g h_{i}=\frac{1}{2} m v_{i}^{2} \\
E_{\text {rebound }}=m g h_{r}=1 / 2 m v_{r}^{2}
\end{gathered}
$$

The height of the drop can be set by adjusting the height of the top base where the double caliper quick release is hung. If there is no visible damage after an impact, a thermal NDE and post-processing sequence is performed to ensure there is no sub-surface damage. The height is then incremented by $2 \mathrm{~cm}$, which adds $2.5 \mathrm{~J}$ to the impact. Due to data recording capabilities of the high-speed camera, $2.5 \mathrm{~J}$ was the smallest increment to give a visible time displacement through the camera recordings. The increments are measured by a measuring tape that runs on the side of the pillar.

The absorbed energy can also be calculated having measured the velocities just before and after the impact using the high-speed camera. Table 6 shows the design properties of the impact test apparatus. A rubber hardness of Shore A50 is used to match the force compression of an inflated bicycle tire.

Table 5 - Impact test specifications

\begin{tabular}{|c|c|}
\hline Specification & Value \\
\hline Impactor mass & $12.432 \mathrm{~kg}$ \\
\hline Impactor dimension & $101.6 \times 76.2 \times 25.4 \mathrm{~mm}$ \\
\hline Impactor shape & Rectangular \\
\hline Rubber hardness & A50 \\
\hline Rubber compression & compression set $40 \%$ \\
\hline Maximum height & $0.92 \mathrm{~m}$ \\
\hline Maximum impact energy & $112.5 \mathrm{~J}$ \\
\hline Maximum striking & $4.2 \mathrm{~m} / \mathrm{s}$ \\
\hline
\end{tabular}

\subsubsection{Impact test setup}

The full setup of the impact test is illustrated below. The test setup includes the impact test apparatus clamped to a steel vibration resistant floor, an infrared camera - FLIR SC5000 [40], and a high speed camera - MotionPro X3 . The cameras are placed on opposite sides of the wheel so that they are both in line with the point of contact between the impactor and the top of the bicycle rim. There are also 2 x 500-watt halogen lamps placed next to the IR camera for the non-destructive 
pre and post evaluation (NDE) phases. An LED is also used to add lighting for the high-speed camera without adding altering the thermal environment.

The IR camera is on a tripod 0.64 meter away from the rim and at a height of 0.5 meter. The purpose of the IR camera is to monitor the impact event by monitoring the heat dissipation of the impact on the surface of the rim. If there is a sudden increase in temperature, the progression of the damage can be observed. The FLIR Research IR software [41] is used to record the event at the frame rate of 173 frames per second. Using post-processing techniques, the IR camera can also be used to evaluate the structure for internal and barely visible damages. By importing the file into Matlab [42], and applying an image processing algorithm using principle component thermography (PCT), pre and post processing for NDE can be performed. The halogen lamps are used to heat up the surface of the rim for NDE.

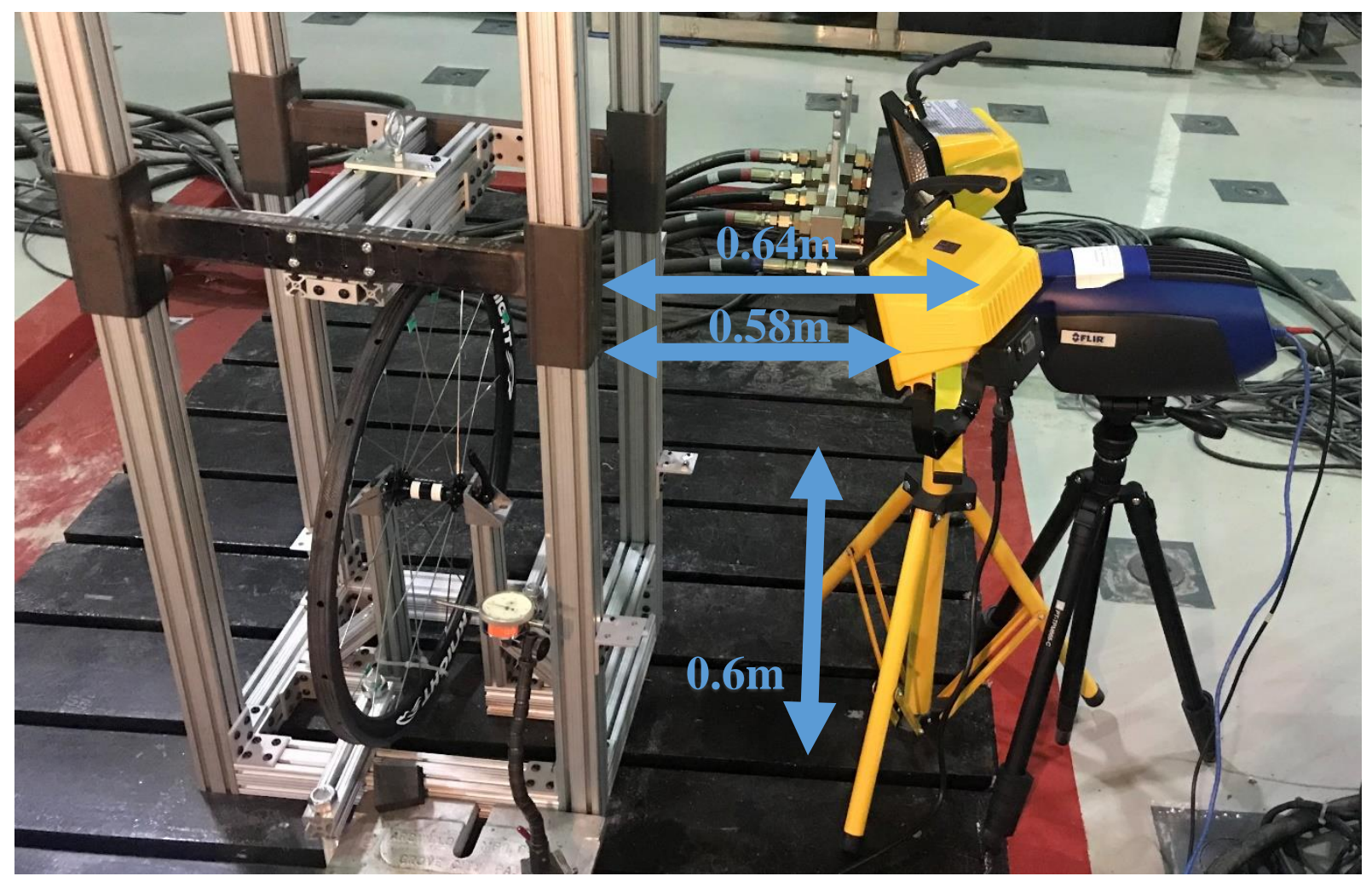

Figure 20 - FLIR SC5000 and halogen lamps setup

The purpose of the high-speed camera is to capture the motion of the impactor and the rim. When the impactor strikes the rim, the compression of the rim can be measured as well as the rebound height of the impactor. The MotionPro X3 is a monochromatic camera, with a maximum recording frequency of $1000 \mathrm{~Hz}$. For this test, $500 \mathrm{~Hz}$ was selected as the recording frequency due 
to the lighting conditions. This frequency creates $2.0 \mathrm{~ms}$ timestamps, which has the resolution to capture the low velocity impacts. The IDT MotionStudio x64 video acquisition software was used to collect the recordings. An onscreen ruler calibrated with the measuring tape on the pillar was used to measure the displacements of the impactor and rim across each frame.

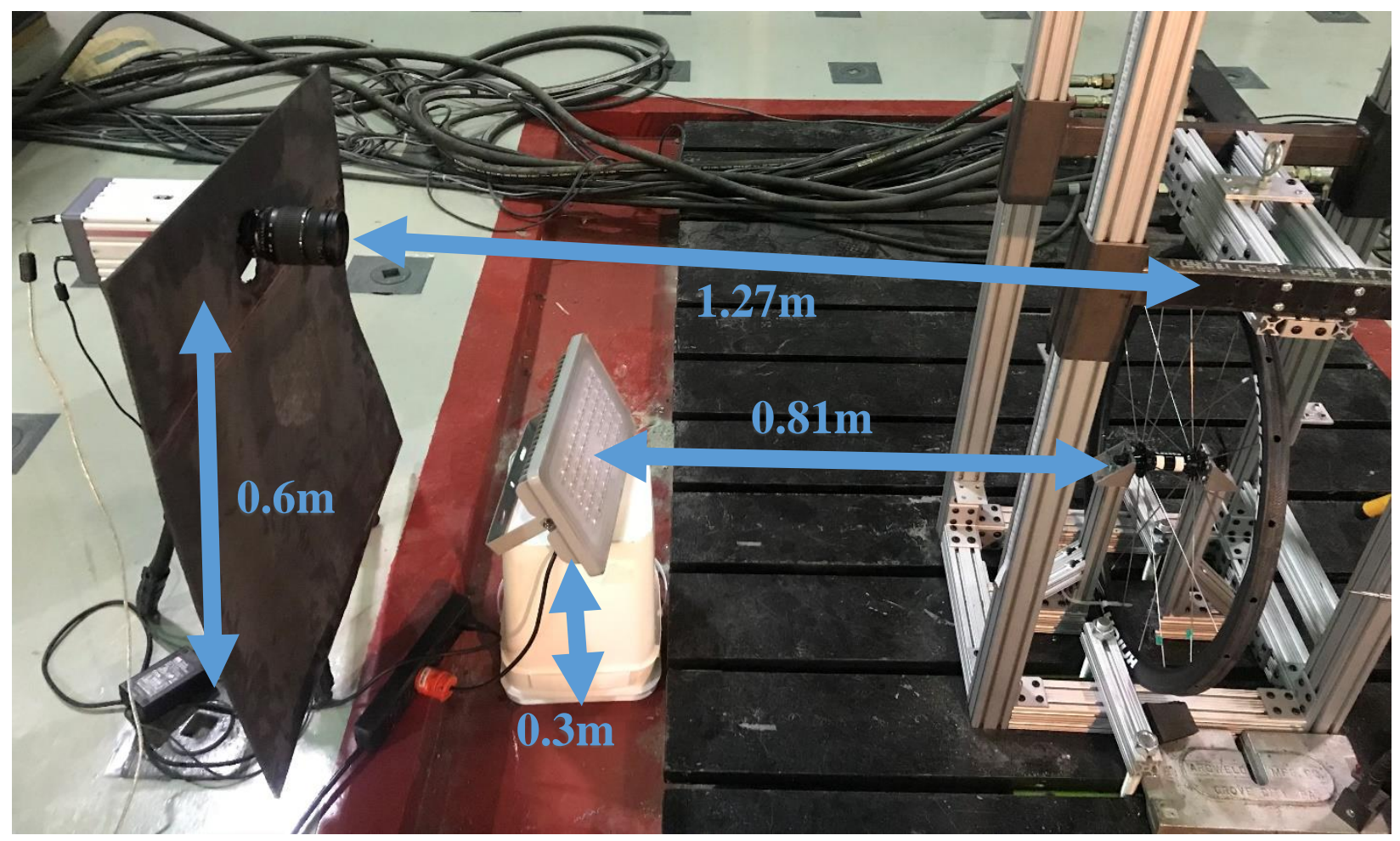

Figure 21 - High speed camera and LED illumination setup

The impactor is held by an eye bolt hung on a double caliper quick release mechanism. This clip ensures that when the impactor is released, it falls straight minimizing the potential of friction between the guide rails and slides. The Kong quick release has a load bearing capability of $25 \mathrm{kN}$. 


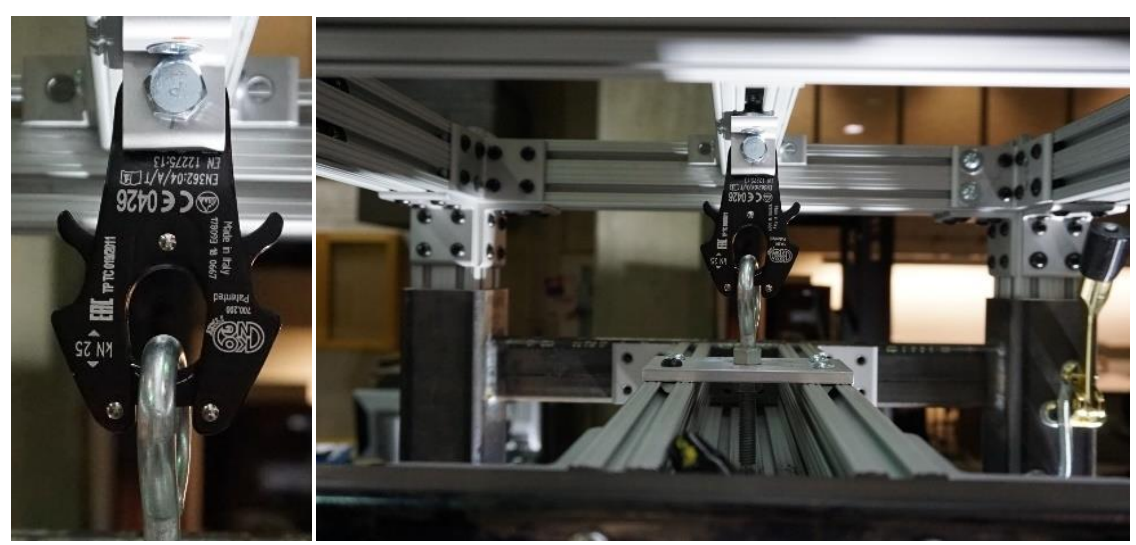

Figure 22 - Double caliper quick release

After the impact, it is necessary for the wheel to be guarded so that a secondary hit on the wheel from the impactor does not occur. For this a stopping mechanism was designed and installed. This mechanism consists of a spring-loaded stopper. Before impact, the stopper is in a loaded vertical up position. After impact, the sudden change in momentum releases the stopper into the vertical down position. As the impactor is settling post rebound, the length of the stopper ensures it rests on a suspended platform from the frame. This ensures that the impactor is physically constrained from hitting the wheel a second time.
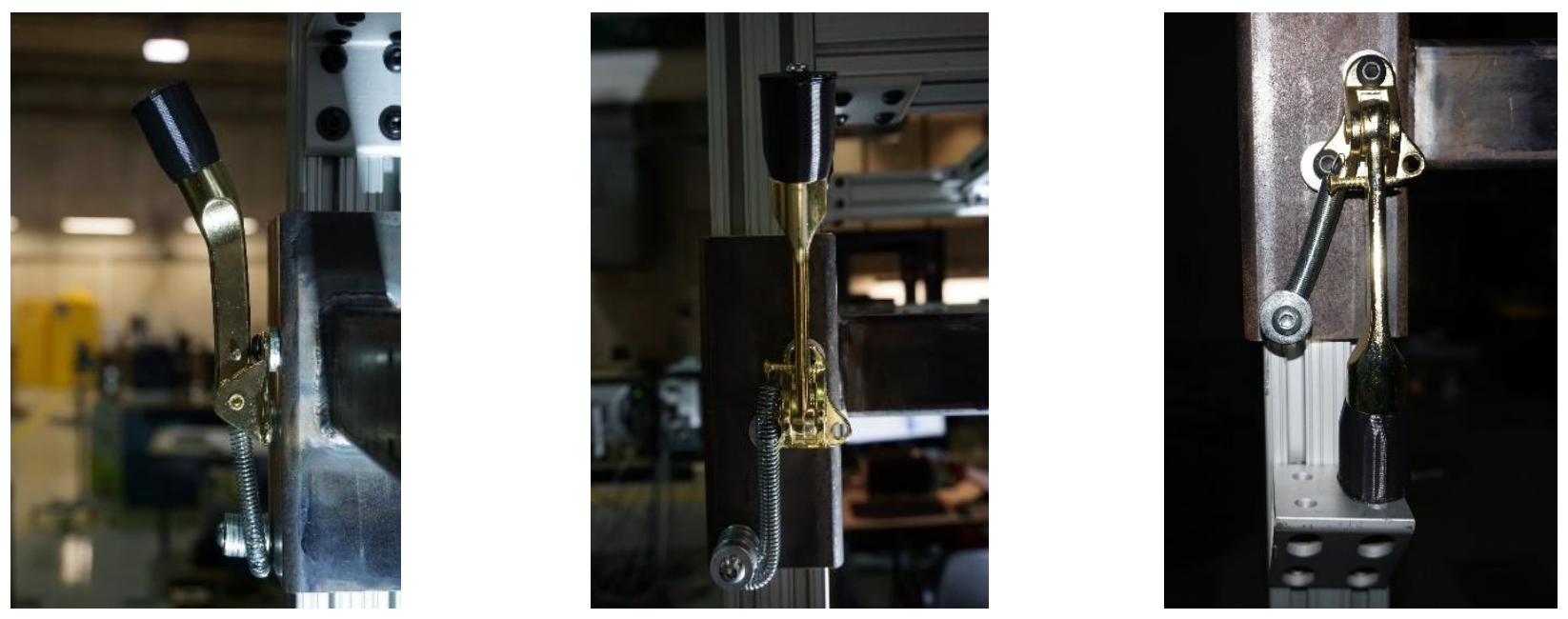

Figure 23 - Spring loaded impactor stop (a) side (b) front pre-impact position (c) front post-impact position

Table 7 shows the error in the impact apparatus through friction. The data is gathered from the all the trails run in the experiment. Although there are some losses between $72.5 \mathrm{~J}$ and $80 \mathrm{~J}$, they can be ignored in this test. 
Table 6 - Jig validation - Impact velocity actual vs theoretical

\begin{tabular}{|c|c|c|c|}
\hline Einput & $\begin{array}{c}v_{i, \text { theoretical }} \\
(\mathrm{m} / \mathrm{s})\end{array}$ & $v_{\text {i,actual }}(\mathrm{m} / \mathrm{s})$ & error $\%$ \\
\hline 55.00 & 2.97 & 2.98 & 0.11 \\
\hline 57.50 & 3.04 & 3.05 & 0.93 \\
\hline 60.00 & 3.11 & 3.11 & 0.08 \\
\hline 62.50 & 3.17 & 3.16 & 1.26 \\
\hline 65.00 & 3.23 & 3.25 & 1.63 \\
\hline 67.50 & 3.30 & 3.29 & 0.79 \\
\hline 70.00 & 3.36 & 3.35 & 0.80 \\
\hline 72.50 & 3.42 & 3.37 & 4.85 \\
\hline 75.00 & 3.47 & 3.47 & 0.13 \\
\hline 77.50 & 3.53 & 3.50 & 3.10 \\
\hline 80.00 & 3.59 & 3.55 & 3.75 \\
\hline
\end{tabular}

\subsection{Impact testing - Design of experiment}

There are many design variables in a bicycle wheel that affect the radial-flexural response of the rim. In this test, many of the variables are constant since only a single wheel model is being investigated. However, there are various configurations in which a wheel can be built. The rim comes in a configuration of 20 and 24 stainless steel spokes which are $1.6 \mathrm{~mm}$ in diameter. The radial spoke pattern is chosen as it provides the maximum radial stiffness [11].

Figure 24 illustrates the design parameters of the bicycle wheel and highlighted in yellow are the parameters investigated for their influence on this test. 


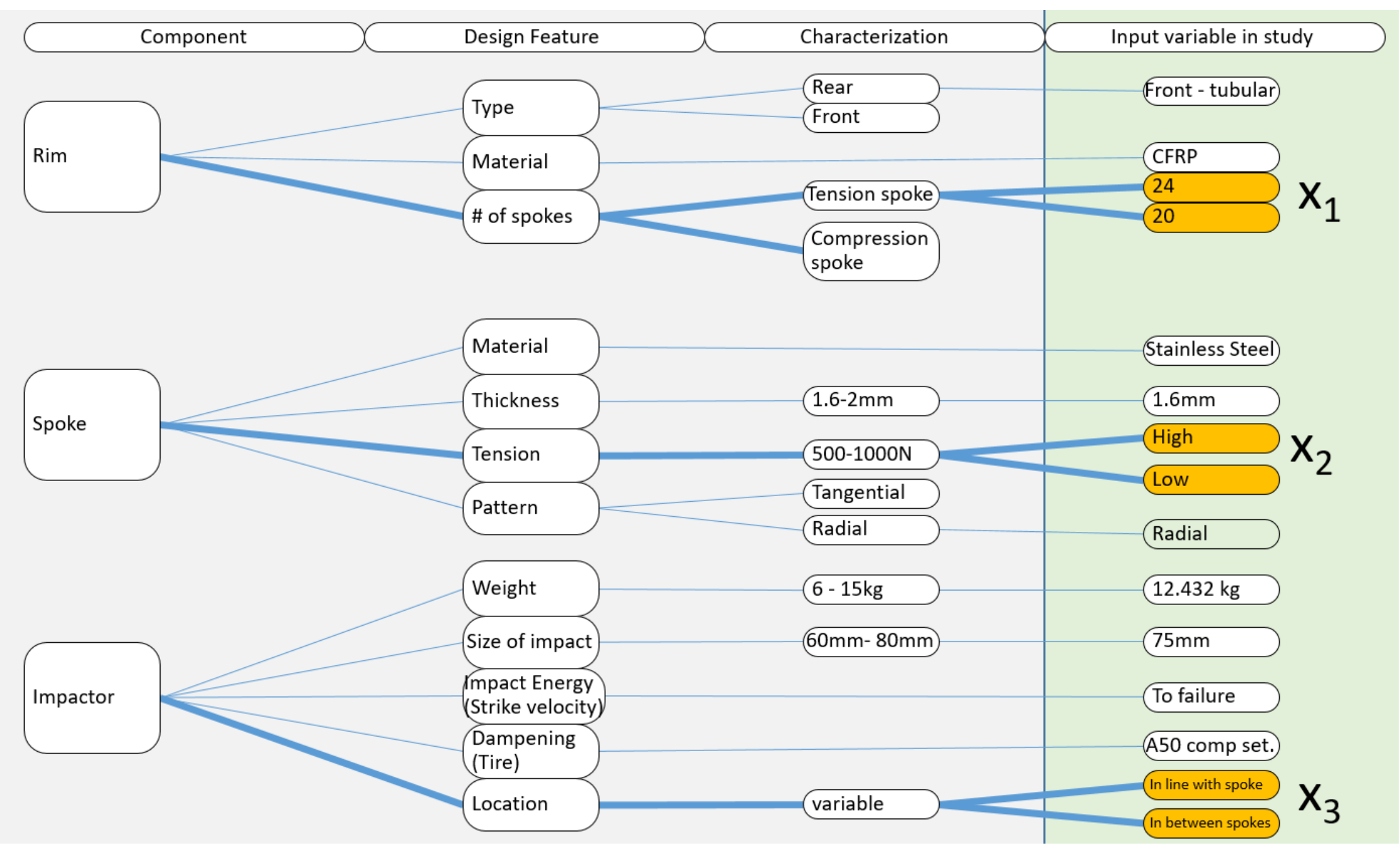

Figure 24 - Design Parameters of Bicycle Wheel and Impact test 
The three variables looked at in this study are spoke pre-tension, impact location, and number of spokes. When a spoke is directly under the applied load, it was found that the deflection of the wheel was less than when the load was applied in the between spokes [9]. Thus impact location was decided to be investigated to see if there is varying impact tolerance along the rim.
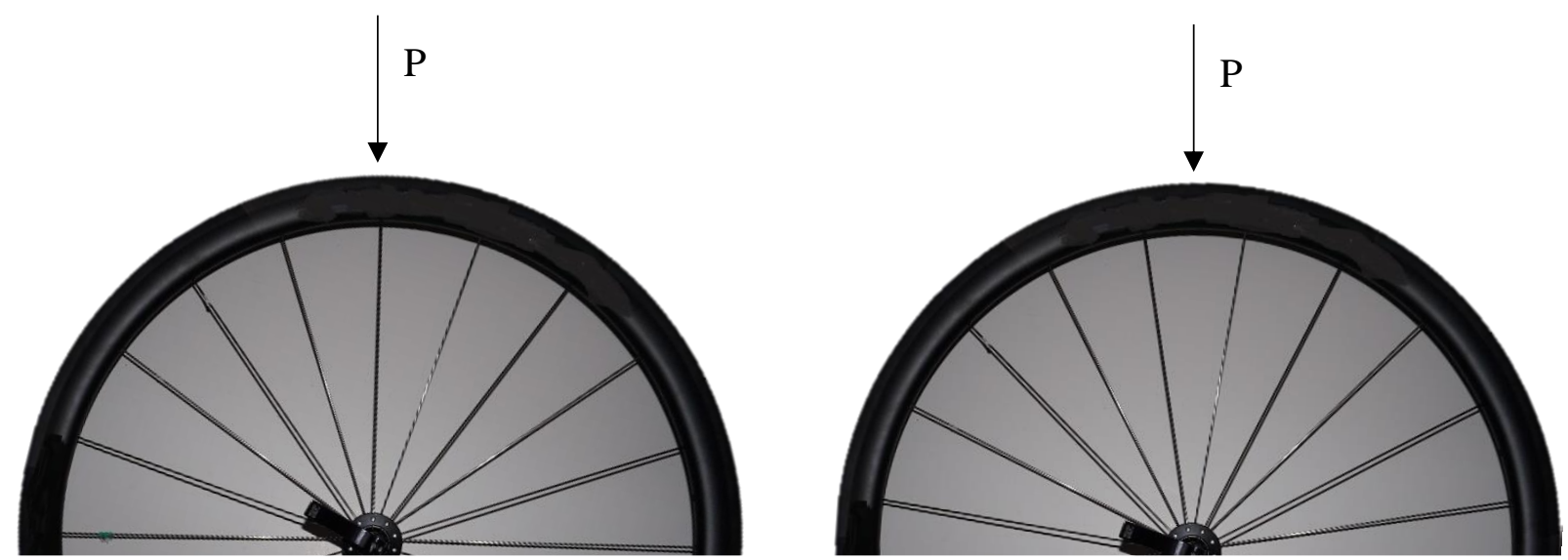

Figure 25 - Loading locations for impact test

Spoke tension in a bicycle wheel must be high enough to prevent the rim from going too slack, however it must not be too high that the induced hoop compression forces create nipple pullout or wheel buckling. From Fords recent studies, it was found that in addition compressive forces faced by the spokes directly under the load on a bicycle wheel, the neighboring spokes increase in tension to balance the compressive forces [13]. If the pretension is high enough that the impact load can pull the spokes over the elastic limit, high internal strain energy occurs, and in the case of carbon fiber wheels, it can initiate fracture. The spoke tension will be also being monitored in successive impacts to see if the tension is loosening. Spoke tension can be measured by a Park Tool Tensionmeter that is pictured below. 


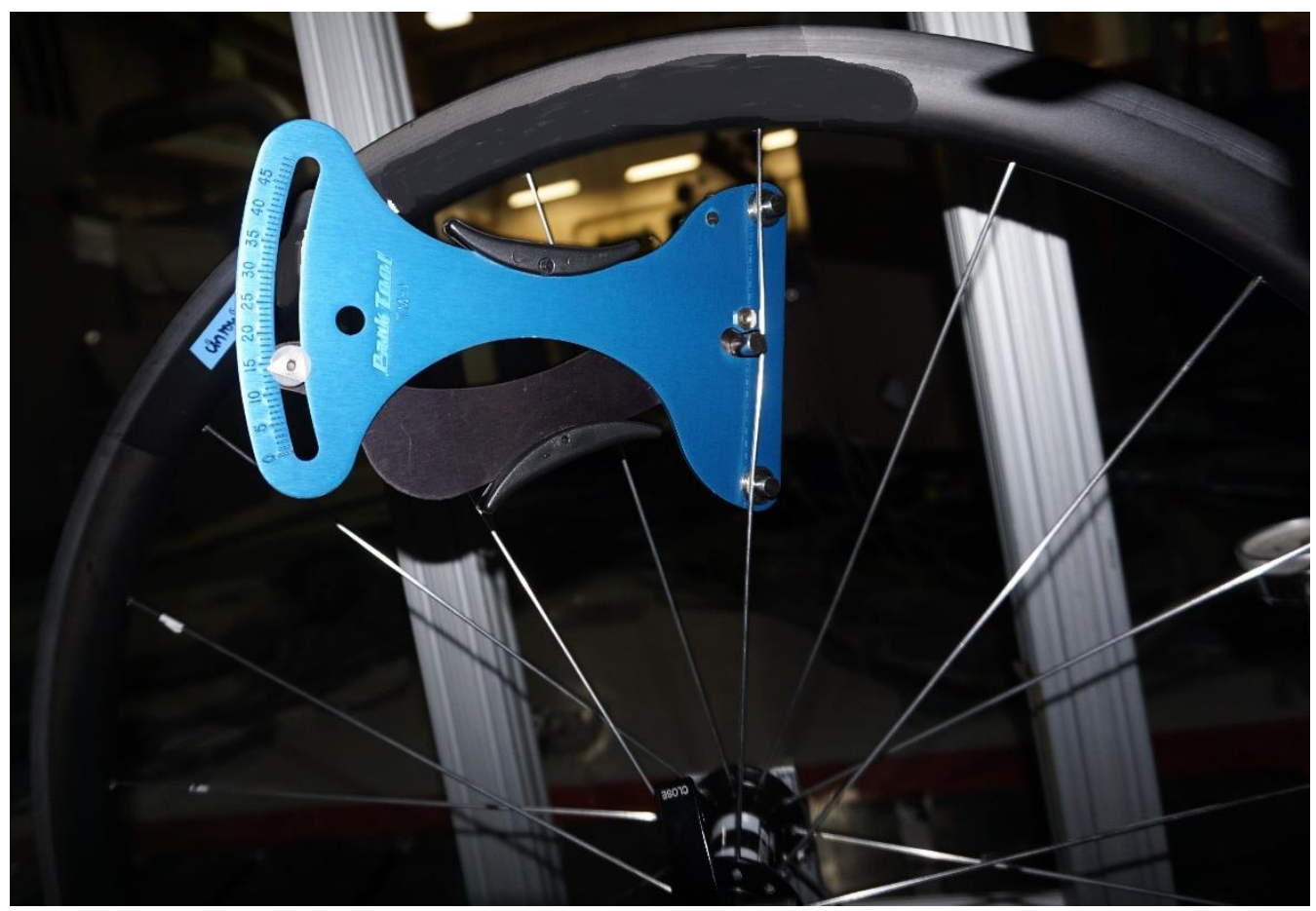

Figure 26 - Spoke tensionmeter reading

When building a wheel, it is necessary to ensure that the wheel is rolling with minimal lateral or radial displacement. This process is known as wheel truing and ensures that the distribution of spoke pretension around the wheel is well balanced. For high performance wheel building, the acceptable true in the lateral and radial directions is $1 \mathrm{~mm}$. This was measured by the dial gage setup shown in Figure 27.
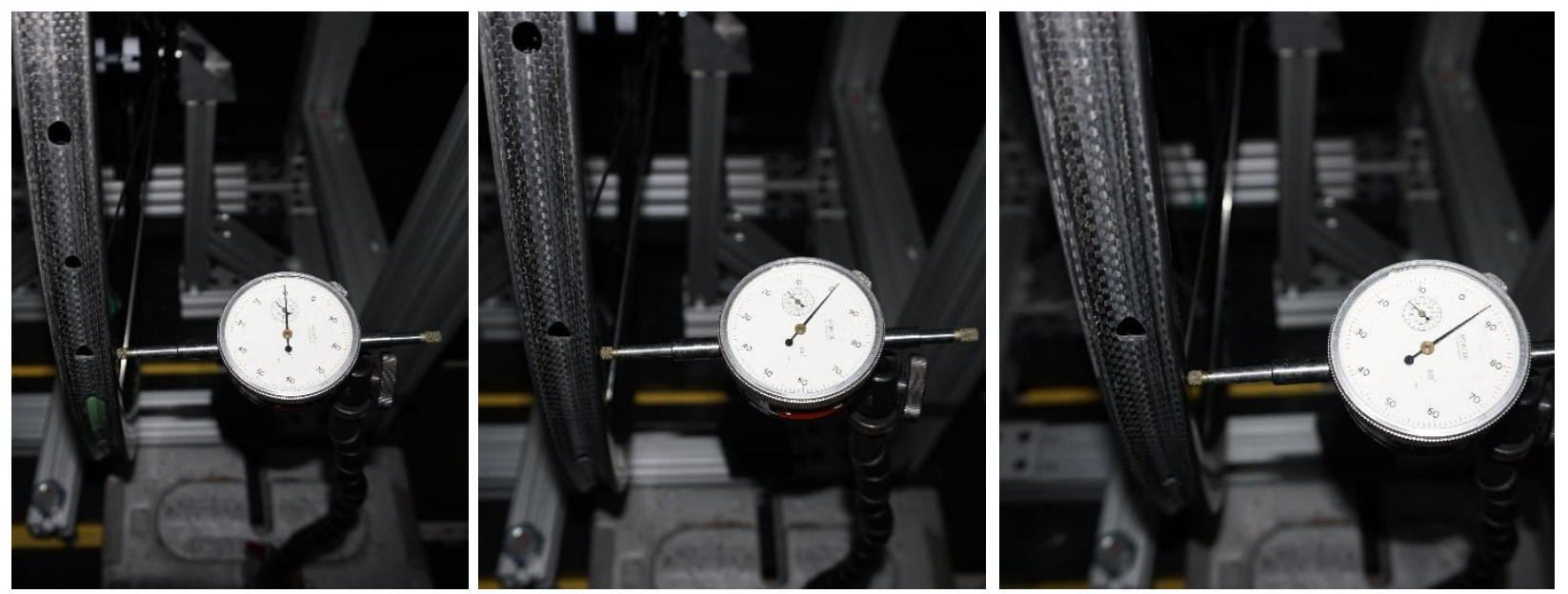

Figure 27 - Wheel truing dial gage (a) left true (b) center true (c) right true 
In order to characterize the impact toughness of the wheel 3 data points will be measured. This includes radial rim deflection, the energy threshold that initiates micro failure, and the spoke tension post impact. With this information the following text matrix has been developed.

Table 7 - Test matrix

\begin{tabular}{|c|c|c|c|c|}
\hline Wheel & $\begin{array}{c}\text { Spokes } \\
(\mathbf{x 1})\end{array}$ & $\begin{array}{c}\text { Spoke } \\
\text { tension (x2) }\end{array}$ & $\begin{array}{c}\text { Impact } \\
\text { location (x3) }\end{array}$ & $\begin{array}{c}\text { \# of } \\
\text { Trials }\end{array}$ \\
\hline 1 & 24 & 20 & -1 & 3 \\
\hline 2 & 24 & 20 & 1 & 3 \\
\hline 3 & 24 & 15 & -1 & 3 \\
\hline 4 & 24 & 15 & 1 & 3 \\
\hline 5 & 20 & 20 & -1 & 3 \\
\hline 6 & 20 & 20 & 1 & 3 \\
\hline 7 & 20 & 15 & -1 & 3 \\
\hline 8 & 20 & 15 & 1 & 3 \\
\hline
\end{tabular}

** Impact location 1 refers to hits radially in-line with a spoke, and -1 indicates in between 2 spokes.

\subsection{Impact testing procedure}

From past studies it was found that, "95\% of the stress from a load in a wheel take place around 4-5 spokes closest to the contact, which is known as the load affected zone" [43]. Due to a limited number of wheels available for testing, 3 impact locations were designated on each wheel (Figure 28). This means that on the 20 spoke wheels, an impact was separated by 7 spokes, and on the 24 spokes wheels they were separated by 8 spokes. Since the impacts were then performed on the same spot within each segment, the impact testing procedure needed a precise inspection 
protocol to rule out any manufacturing defects or progressive damage on the wheel. Using the IR

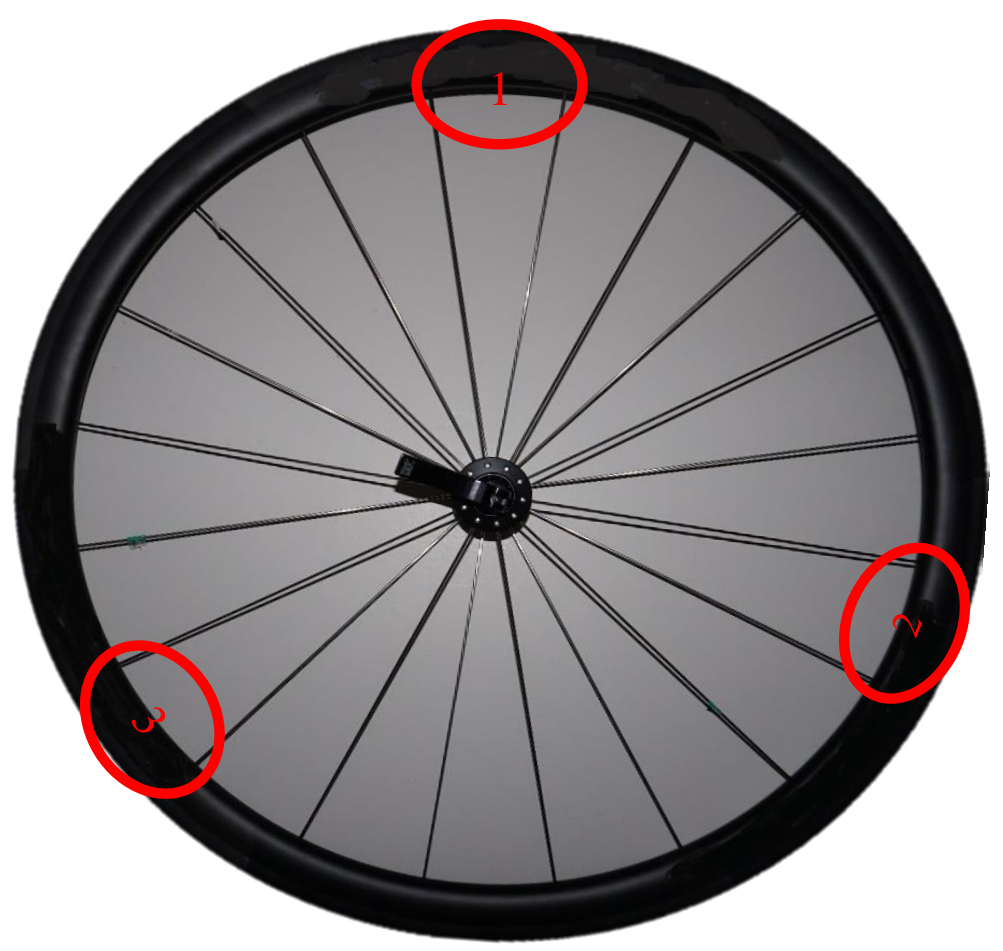

Figure 28 - Impact locations on wheel under test

PCT technique, the wheels were monitored before and after every impact until failure.

The following are the steps used to perform this experiment

1. Assemble cameras and equipment into correct positions. Check focus and ensure connection with Research IR+ and Motion Studio data capturing software

2. Perform wheel building process. Ensure spoke tension, and wheel truing is to performance standard.

3. Mount wheel and inspect for manufacturing defects using IR camera or pre-impact inspection.

4. Mount release mechanism to desired height and mount weight. Ensure catch mechanism is in the loaded-up position.

5. When ready, release impactor, ensuring high speed and IR camera are triggered.

6. Collect data and perform post impact inspection.

7. If post impaction inspection is a PASS and there is no visible damage, repeat steps 4 to 7 and increment impact height by $2.5 \mathrm{~J}$ until failure. 


\subsection{Validation of Pre-test/Post-processing Non-Destructive Inspection}

Composite materials are susceptible to internal damage through impacts. These damages include invisible cracks and delamination which are not visible and lower the mechanical strength of the structure. Active infrared thermography along with principal component analysis has been setup for non-destructive inspection. The procedure set by Ruben et al. [28] for NDE was implemented for this test.

In Figure 29, an inflammation is shown on a wheel after an impact and the corresponding temperatures of the 4 points throughout the impact are shown in Figure 30. Point 4 which is in the impact region shows a jump of $2^{\circ} \mathrm{C}$ whereas the other 3 points have no change. (Note: due to the
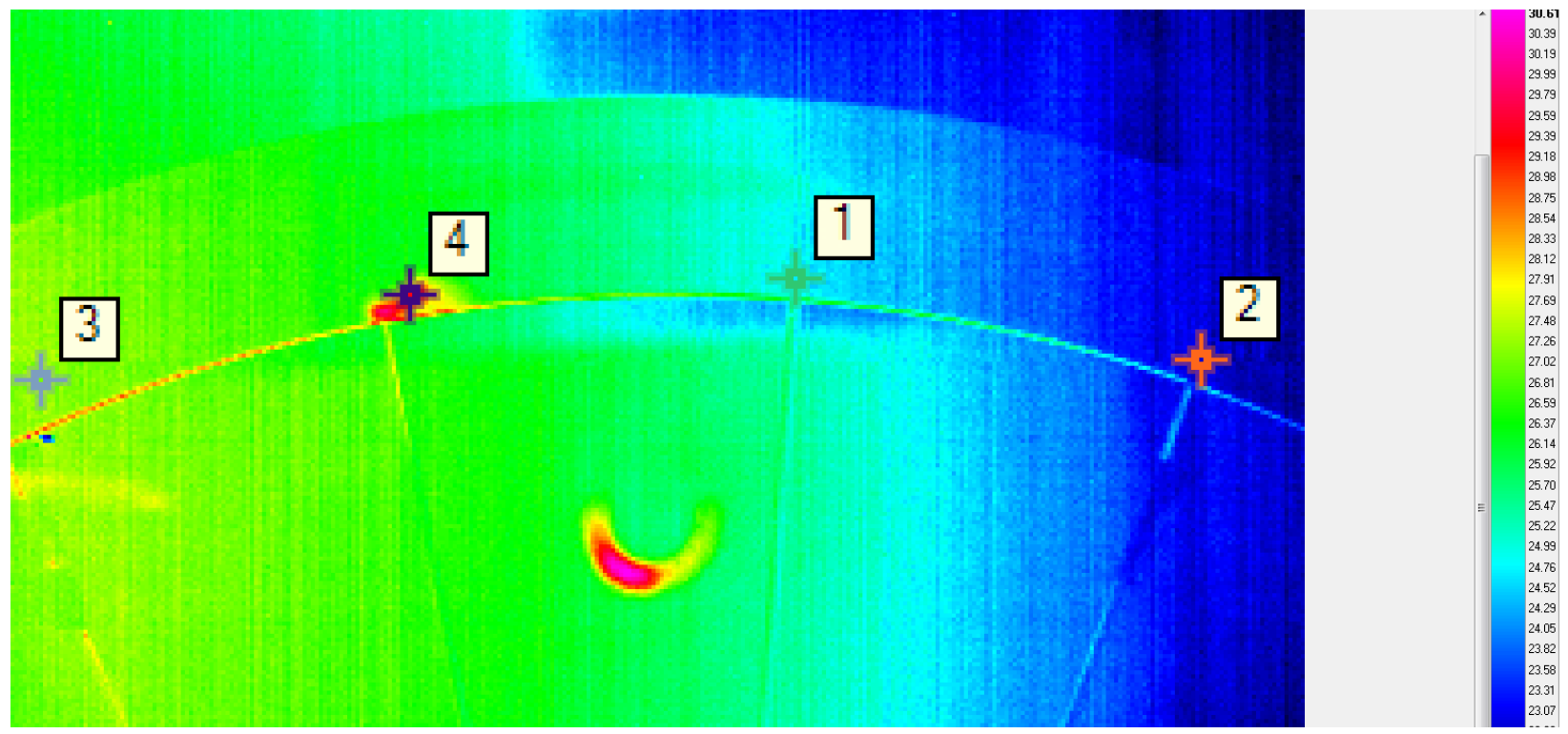

Figure 30 - IR image of test wheel

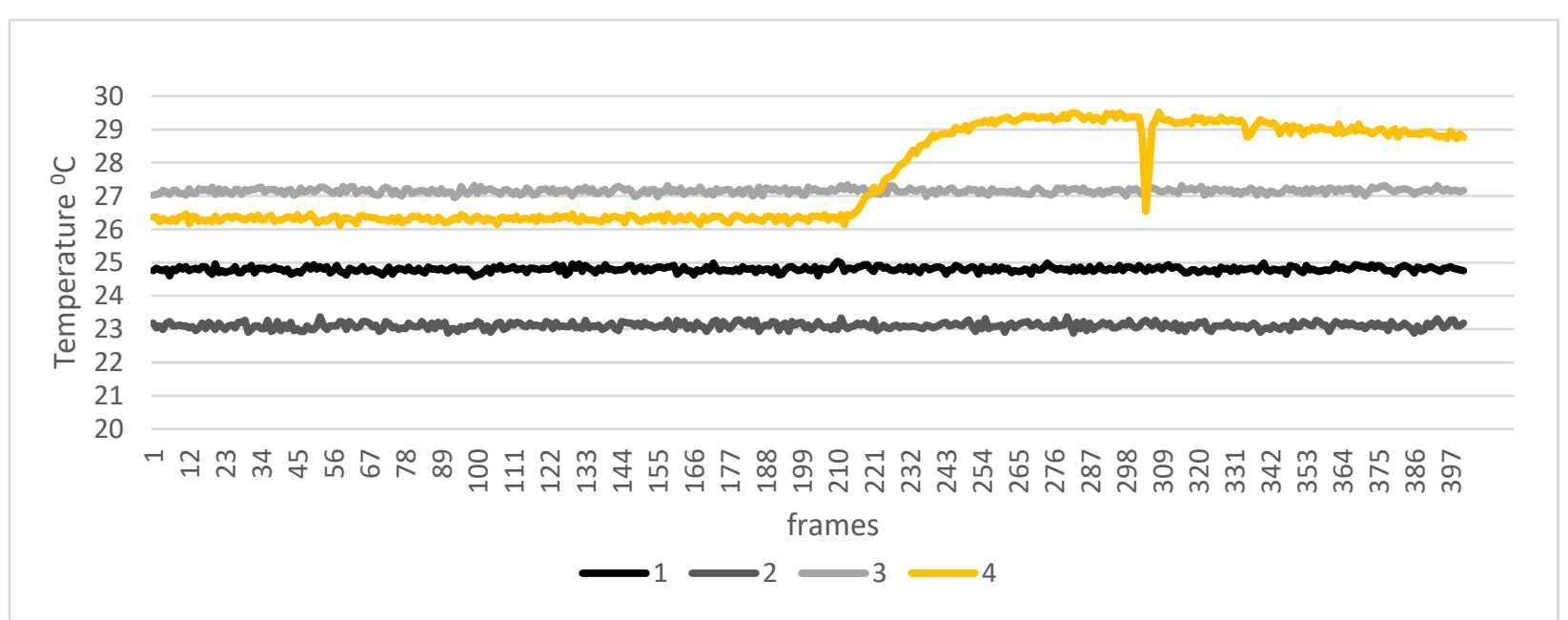

Figure 29 - Thermal monitoring of impact on wheel 
physical location of the test and room heating system there is a large thermal non-uniformity in steady state temperature between points 1-4). The heat release at point 4 has had some physical change and the IR camera has successfully captured the live heat release. Figure 32a shows the segment of the wheel post impact, and there is no visible damage on the surface.

The thermal sequence was then performed in which the temperature profile across the same 4 points were monitored over a 30 second warm up and 30 second cool down period. Points 1-3 have similar temperature ramp up and cool down rates and average an increase of $2.1^{\circ} \mathrm{C}$. Point 4 however has the smallest change in temperature change as it rises $1.7^{\circ} \mathrm{C}$ and the slowest cool down rate.

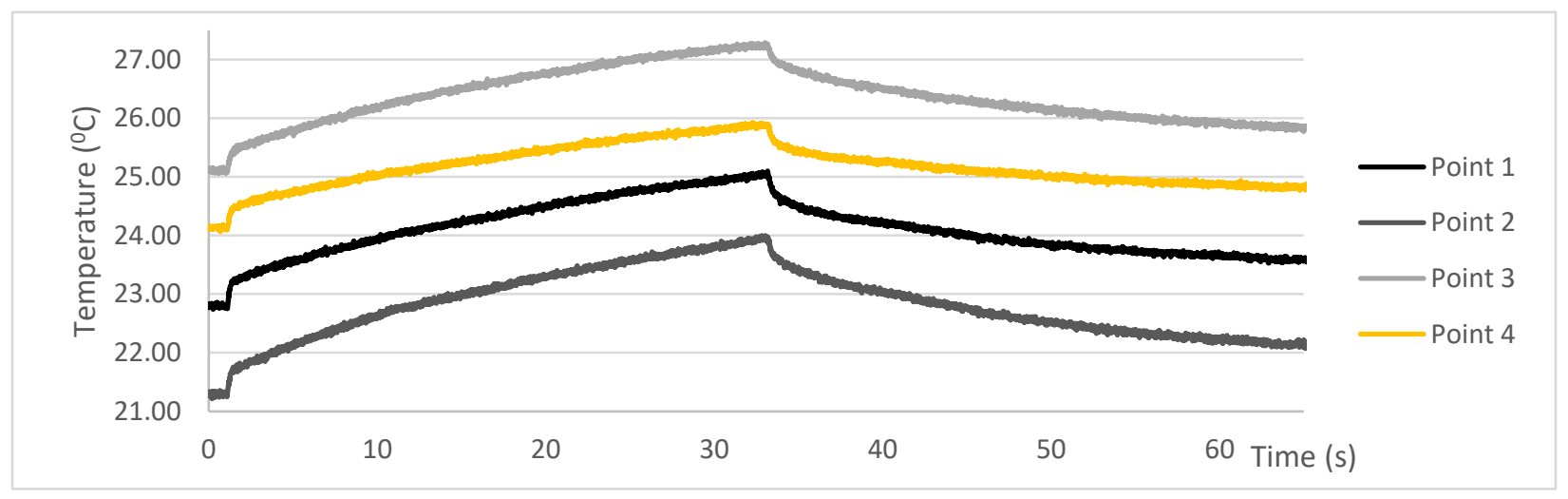

Figure 31 - IR PCT thermal sequence of test wheel 
After performing a PCT post-processing sequence, the image in Figure 32b is obtained. At the point highlighted there is a clear discrepancy in the thermal variation at point 4 . The wheel was then sectioned at point 4 and the subsurface crack was confirmed.

a

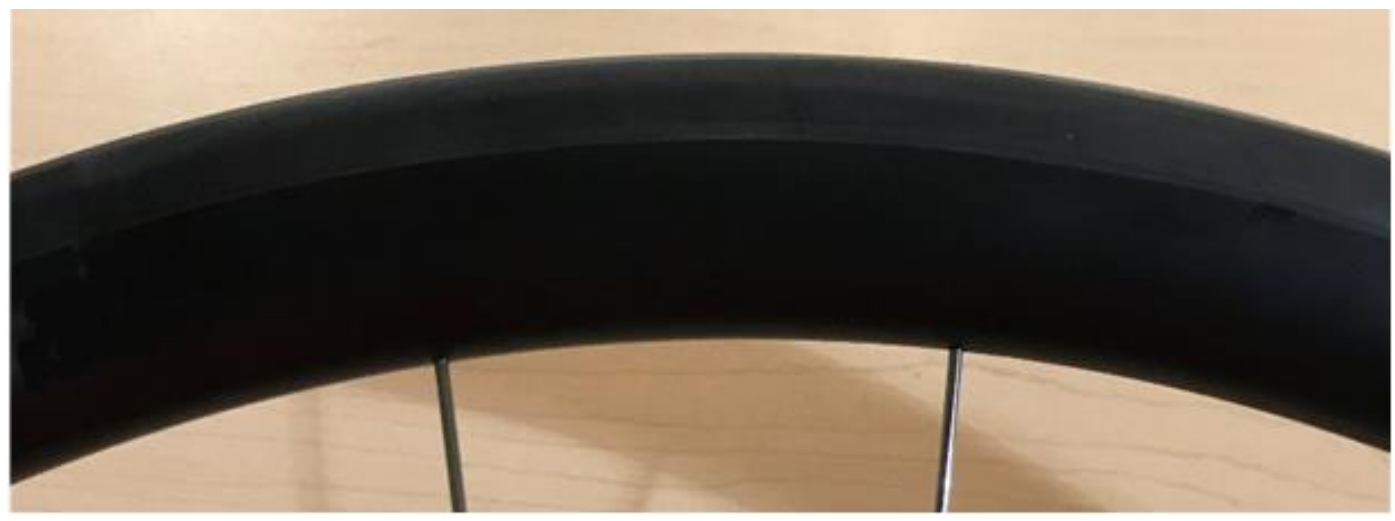

b

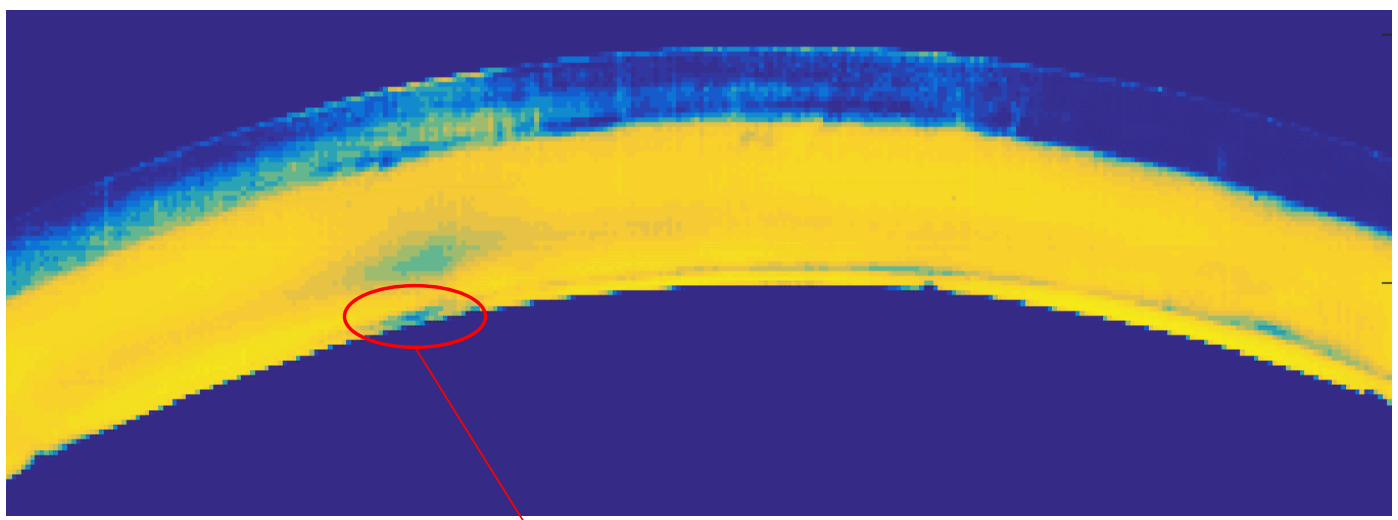

c

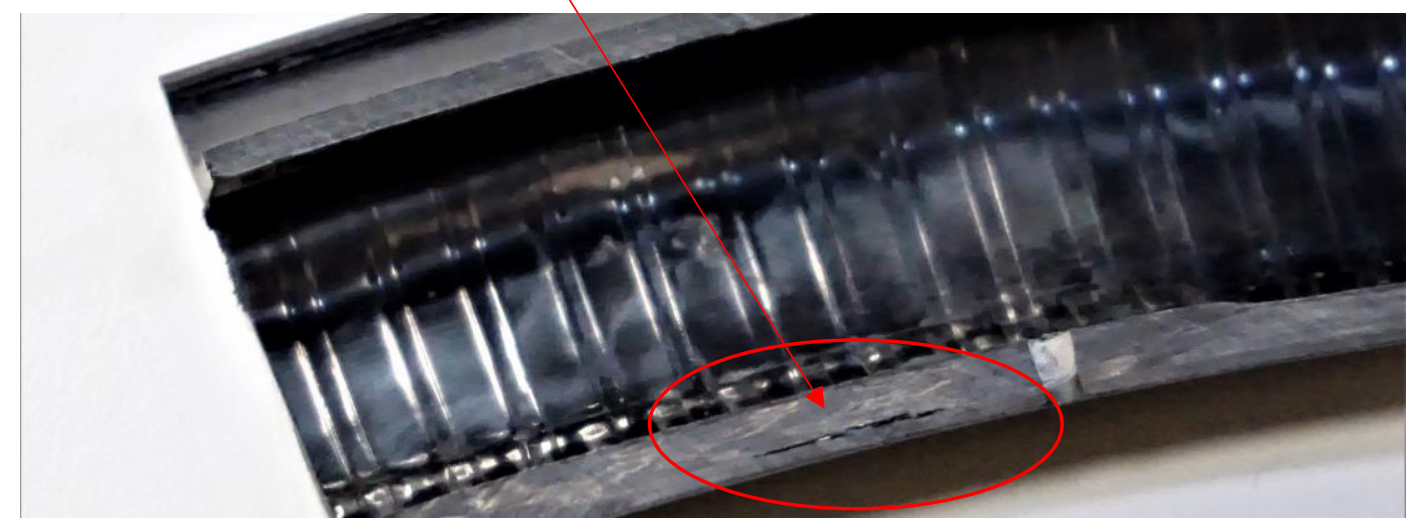

Figure 32 - (a) surface of wheel (b) PCT of surface (c) section of wheel in area of point 4 


\section{Chapter 4}

\subsection{Results and discussion}

\subsection{Impact Results}

An equal energy line indicates equality between impact energy and absorbed energy. The gap between the equal energy line indicates unabsorbed energy during impact, and the gap determines energy absorption efficiency of the composite material. Throughout literature, the energy absorption criteria is used to determine a materials impact toughness. In this test, while it is possible to observe the absorbed energy during impact, the energy absorption is divided among the rim and the spokes. Without having instrumented spokes, it is not possible to differentiate between the energy absorbed by the rim and the energy transferred into the spokes. Instead, radial deflection of the rim along with energy absorption is used to normalize the results of each impact test.

In this study an initial impact energy of $57.5 \mathrm{~J}$ was used as per manufacturer recommendation. The impact response Figures 34-57 show the failure threshold of each wheel configuration and the successive tests performed at each location. If a test had passed, a post inspection was performed to ensure no subsurface damage had occurred. If the post inspection had passed, the drop height was incremented next test performed. The wheel would then be rotated to the next location to test for repeatability. The impact energy, energy absorbed, and radial displacement of the wheel was calculated and analyzed. In Figures 34-57, trials highlighted in the red colored zone had failed.

\subsubsection{Wheel 1}

For the 24 spoke wheel with high spoke tension and an impact between spokes, the average failure threshold was $60 \mathrm{~J}$ (Figure 33-35). This was consistent among the three locations. It can be observed that from the 3 failures, the rim failed when it absorbed $31 \mathrm{~J}$ or higher and displaced above $8 \mathrm{~mm}$. When failure initiated, it started from the spoke access hole in the lateral direction. The cracks did not extend to the rim walls. 


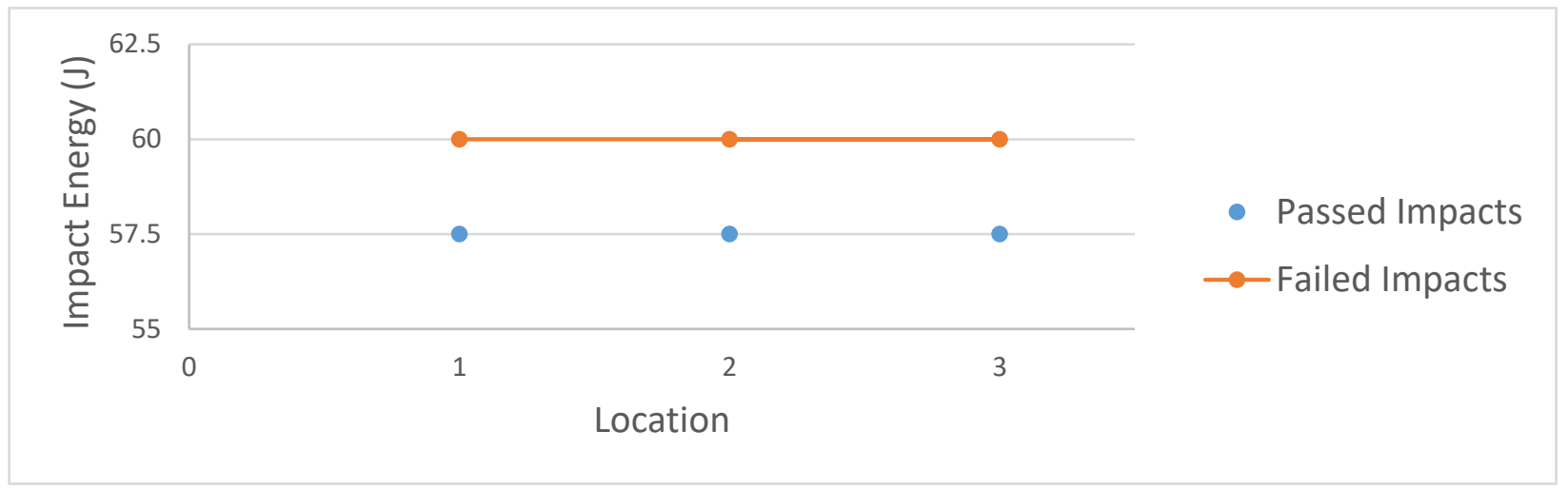

Figure 33 - Impact results of wheel 1 - 24 spoke - high tension - in between spokes

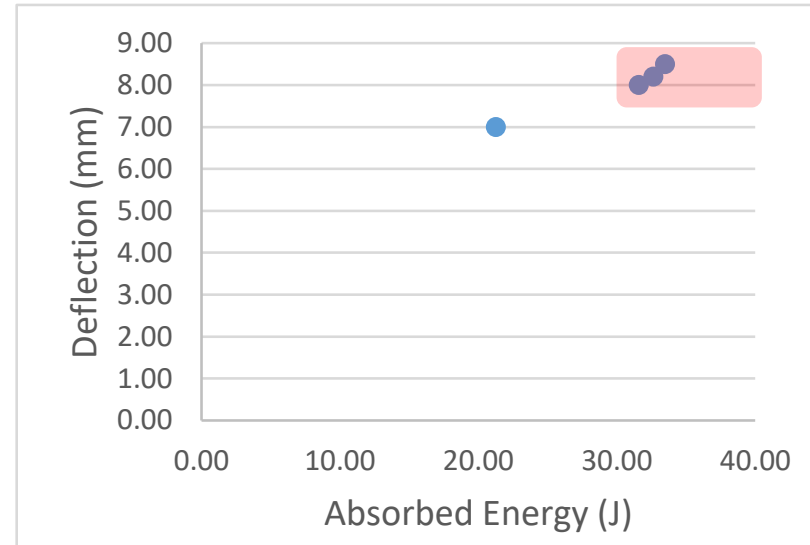

Figure 34 - Absorbed Energy vs Radial Deflection of wheel 1

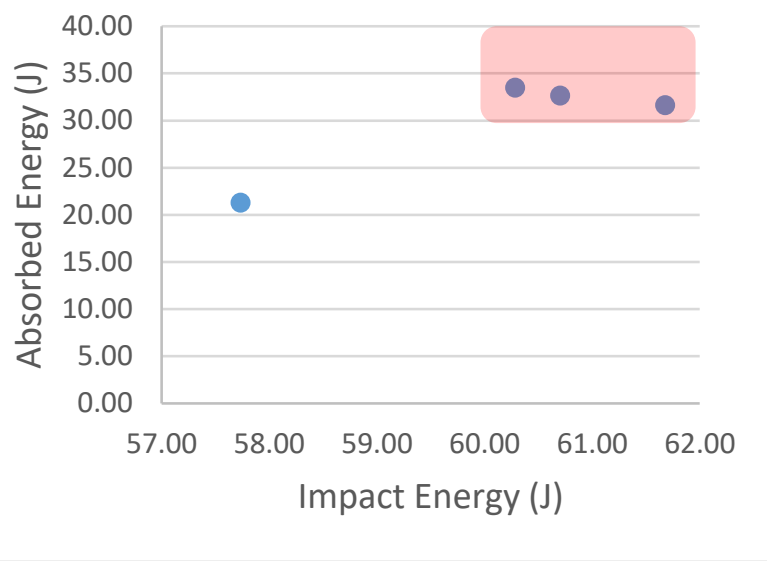

Figure 35 - Impact Energy vs Absorbed Energy of wheel 1

\subsubsection{Wheel 2}

In wheel 2, the configuration was a 24 spoke hole with lower spoke tension and the impact in between the spokes. The impact threshold was slightly lower than wheel 1 with an average of 59.2 $J$, and a minimum impact threshold of $57.5 \mathrm{~J}$ (Figure 36). Although more energy was absorbed and there was a higher radial displacement in wheel 1, wheel 2 exhibited larger cracks. This could be due to variation lower spoke tension. The crack initiation behavior was similar as it started from the spoke access holes and grew to the edges of the rim. 


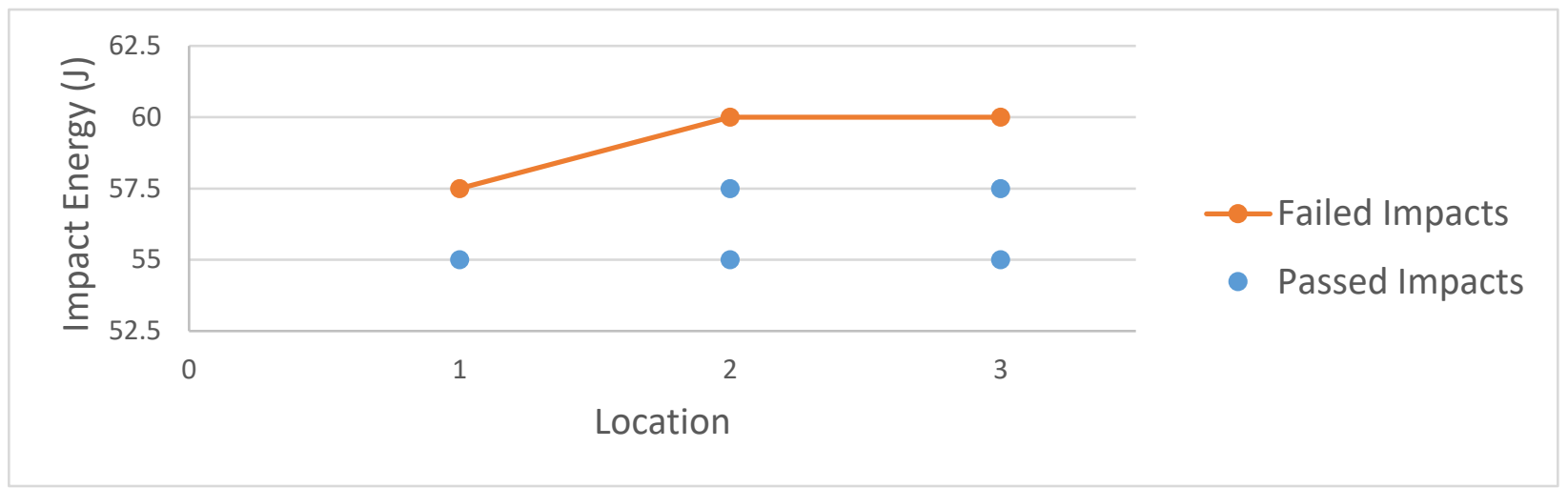

Figure 36 - Impact results of wheel 2 - 24 spoke - low tension - in between spokes

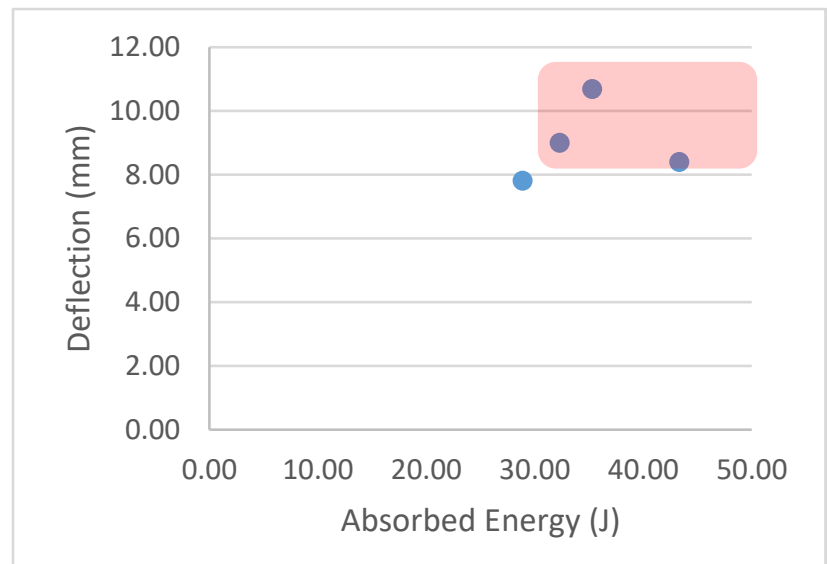

Figure 37 - Absorbed Energy vs Radial Deflection of wheel 2

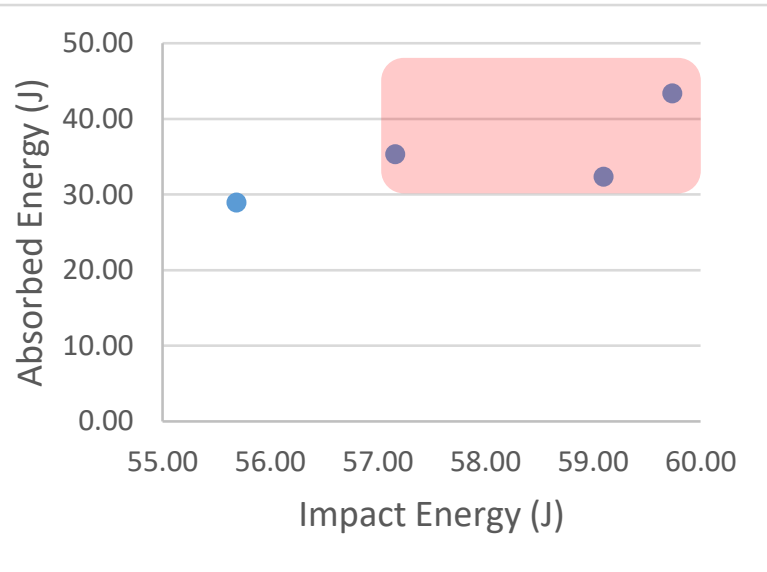

Figure 38 - Impact Energy vs Absorbed Energy of wheel 2

\subsubsection{Wheel 3}

In wheel 3, the weight was dropped in line with the spokes and the failure threshold was significantly larger than the impacts that were in between the spokes. The average impact failure was $69 J$ in which the wheel could absorb up to $34 J$ without failing (Figures 39 and 41). Its maximum radial displacement was also up to $8.6 \mathrm{~mm}$ (Figures 40). However, when failure could be seen, large energy releases created long cracks and very large delamination areas. In this impact, the crack starts at the spoke access hole directly underneath the impactor and propagates to the edge of the rim and down the sidewall. Once it travels down the sidewall, it meets the thinnest segment of the cross section, where 3 plies have tapered off and splits tangentially. 


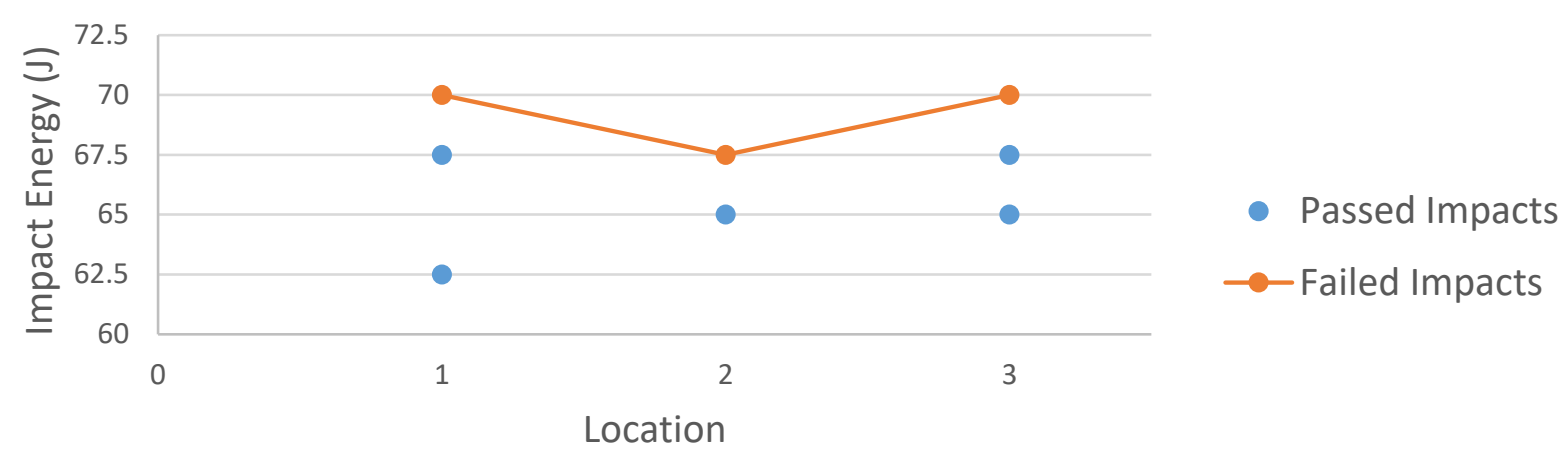

Figure 39 - Impact results of wheel 3 - 24 spoke wheel - high tension - in line

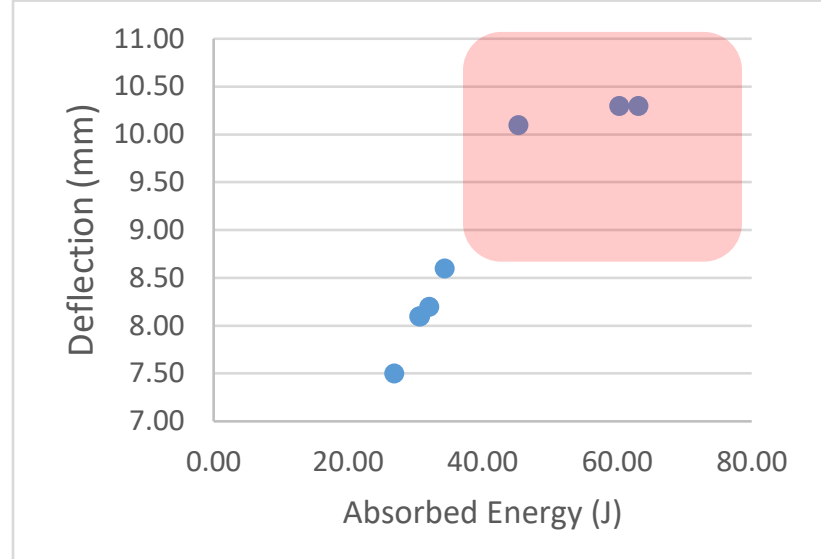

Figure 40 - Absorbed Energy vs Radial Deflection of wheel 3

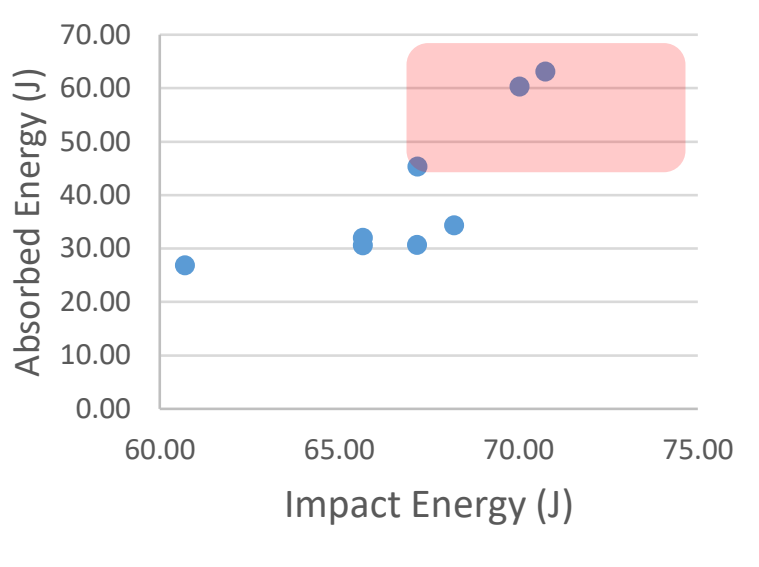

Figure 41 - Impact energy vs Absorbed energy of wheel 3

\subsubsection{Wheel 4}

In wheel 4, the failure energy was lower than wheel 3 but higher than both wheel 1 and wheel 2, where the impacts were in between the spokes. Wheel 4 had an average failure threshold of $61.7 \mathrm{~J}$ (Figure 42) and a maximum radial displacement of $8.6 \mathrm{~mm}$ (Figure 43). A similar crack initiation and propagation pattern to wheel 3 was noticed where it started from the spoke access hole directly underneath and traveled toward the edge and down the sidewall. 


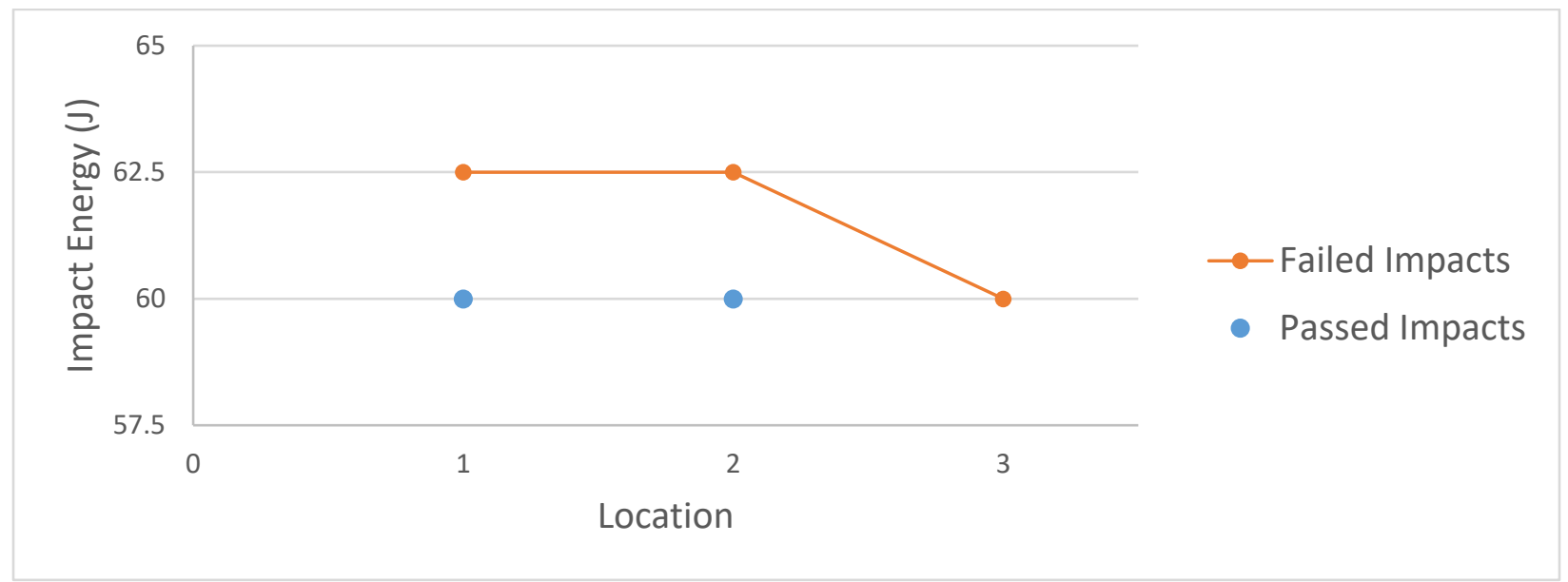

Figure 42 - Impact results of wheel $4-24$ spoke wheel - low tension - in line

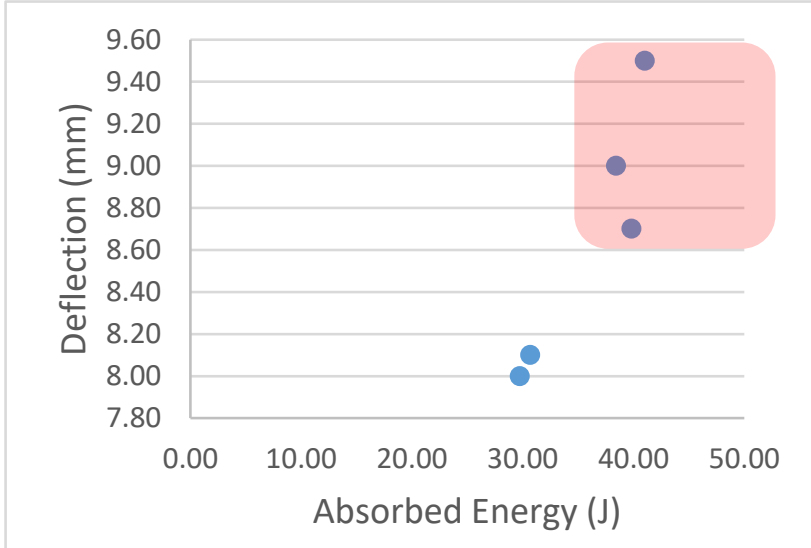

Figure 43 - Absorbed Energy vs Radial Deflection of wheel 4

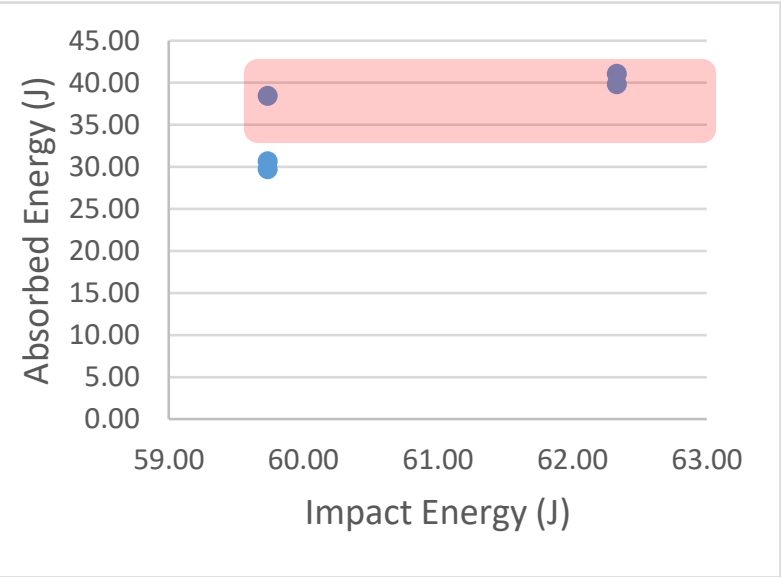

Figure 44 - Impact energy vs Absorbed energy of wheel 4

\subsubsection{Wheel 5}

Wheel 5 was a 20 spoke wheel built with high spoke tension and impacted in between the spokes. This was comparable to wheel 1 which had 24 spokes. In wheel 5, there was 2 failures of $57.5 \mathrm{~J}$ and one of $60 \mathrm{~J}$ (Figure 45). Although one test was slightly higher in failure than wheel 1, it is not clear the effects of the lower spoke count on failure threshold. The cracks, however, were more apparent in wheel 5. This suggests that since more damage was visible, the rim had absorbed more energy. The radial displacement of wheel 5 was a little bit higher in the range of $8.1 \mathrm{~mm}$ (Figure 46). The crack initiation behavior was also very similar in which it started from the spoke access hole. 


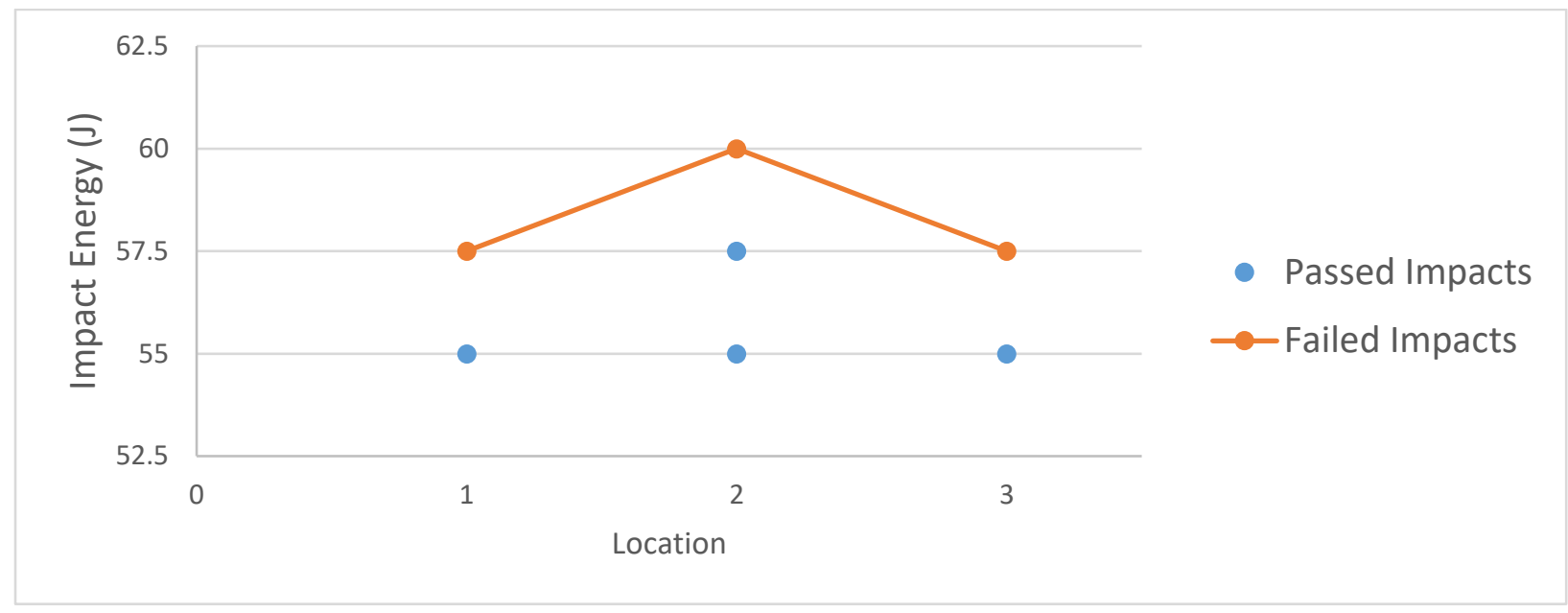

Figure 45 - Impact results of wheel 5 - 20 spoke wheel - high tension - in between

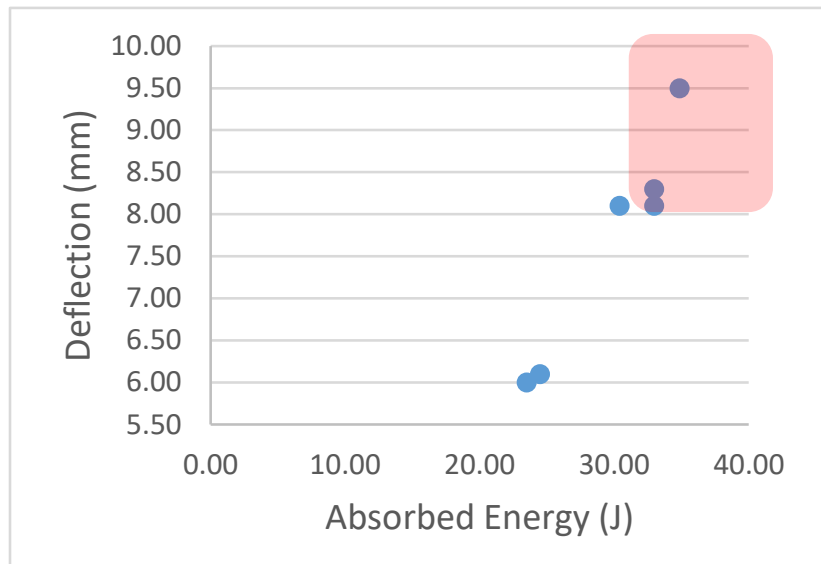

Figure 46 - Absorbed Energy vs Radial Deflection of wheel 5

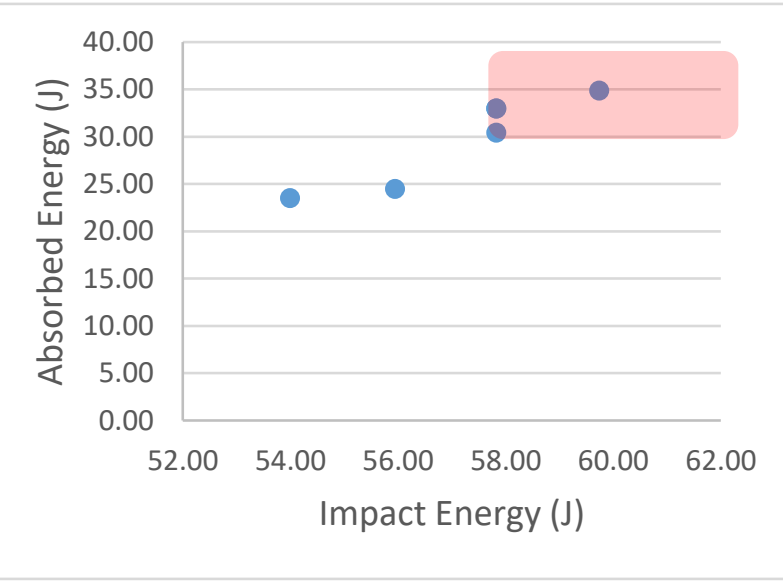

Figure 47 - Impact energy vs Absorbed energy of wheel 5

\subsubsection{Wheel 6}

Wheel 6, which was a 20 spoke wheel with lower spoke tension and an impact in between the spokes, experienced an average failure energy equal to of $58.3 \mathrm{~J}$. It was also comparable to wheel 1, wheel 2, and wheel 5. All four wheels which were loaded in between the spokes failed within the energy levels of 57.5 and $60 J$ (Figure 48). This was consistent between spoke count and spoke tension. The maximum radial displacement before failure was $8.2 \mathrm{~mm}$ (Figure 49). 


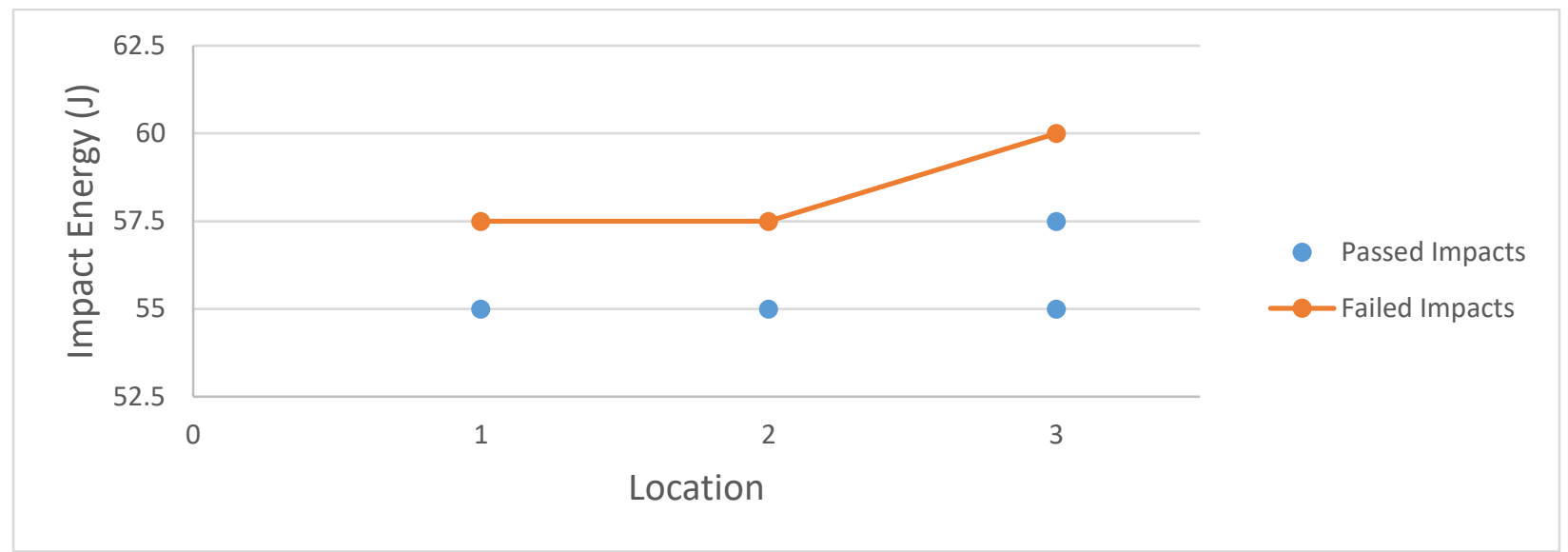

Figure 48 - Impact results of wheel 6 - 20 spoke wheel - low tension - in between

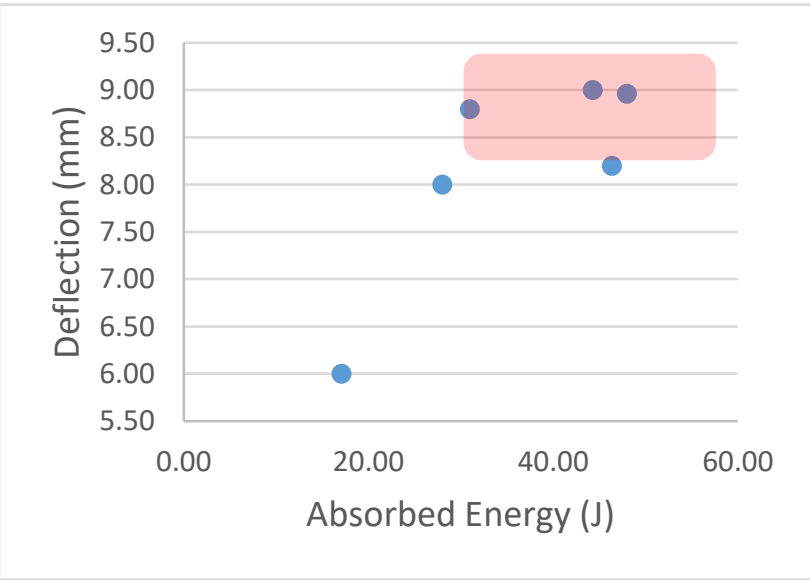

Figure 49 - Absorbed Energy vs Radial Deflection of wheel 6

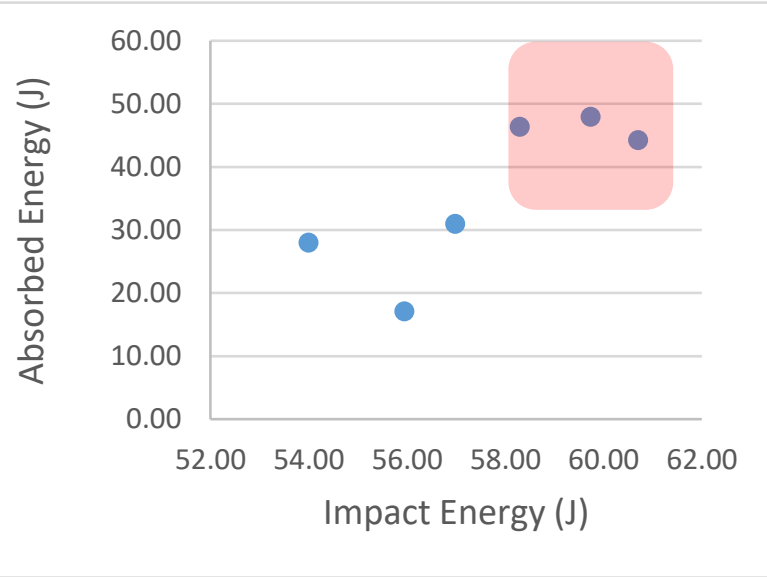

Figure 50 - Impact energy vs Absorbed energy of wheel 6

\subsubsection{Wheel 7}

Wheel 7, which was a 20 spoke wheel built with high spoke tension and the impact loaded in line with spoke, had the highest average impact energy threshold of $76.7 \mathrm{~J}$. Its maximum impact tolerance was $80 \mathrm{~J}$, and minimum was $72.5 \mathrm{~J}$ (Figure 51). It also withstood high radial displacements of up to $8.8 \mathrm{~mm}$ (Figure 52). Although the wheel was able to take such high impacts, when it failed the cracks were recorded as long as $150 \mathrm{~mm}$. 


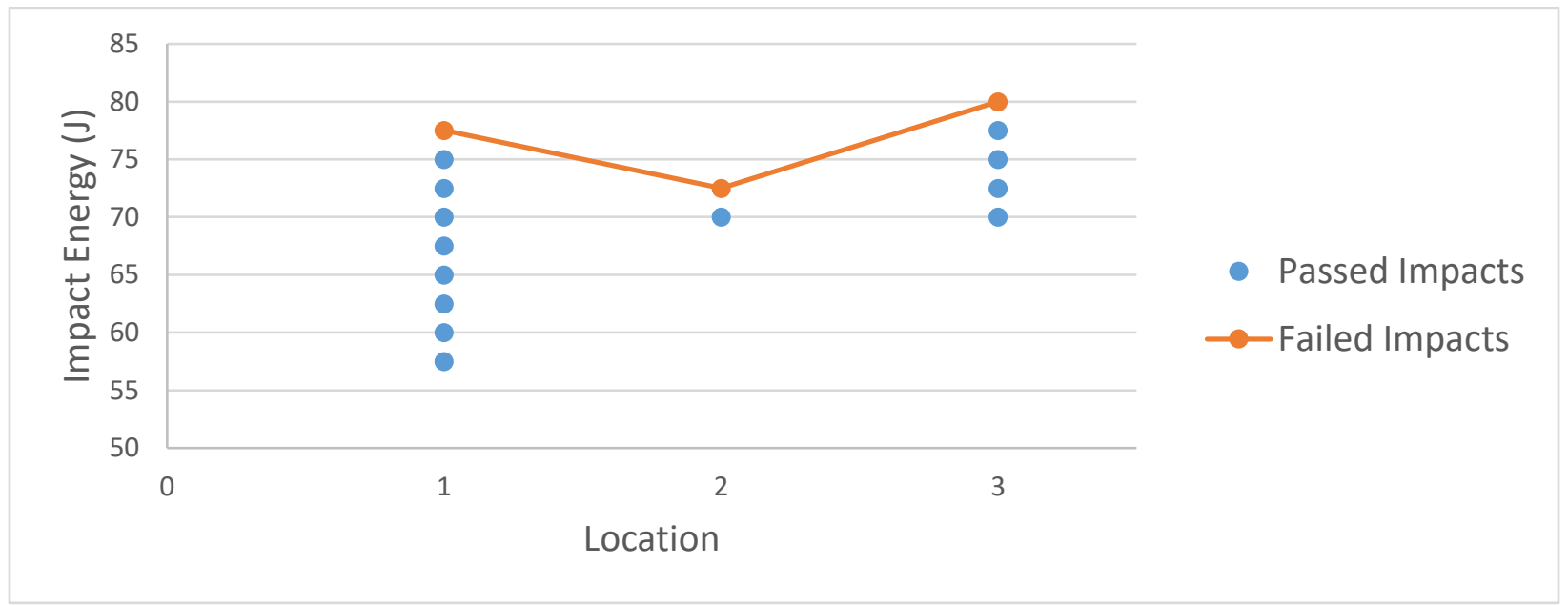

Figure 51 - Impact results of wheel 7 - 20 spoke wheel - high tension - in line

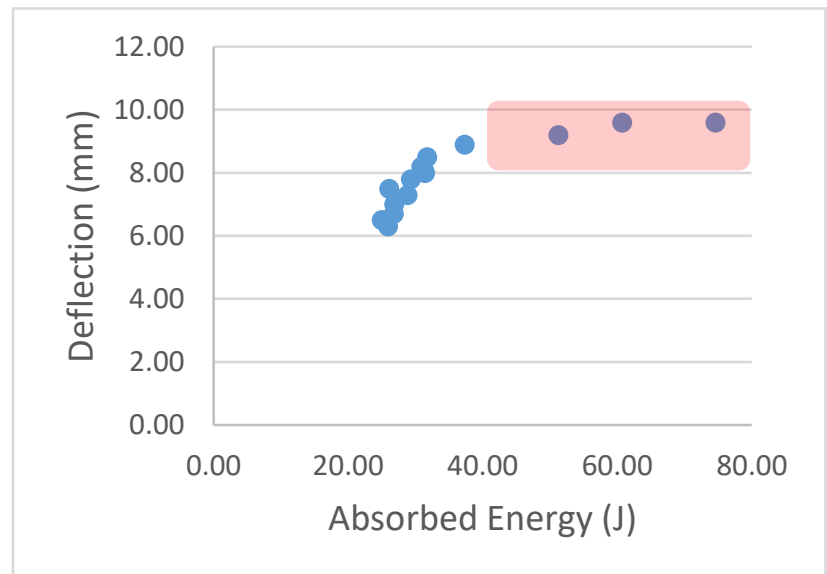

Figure 52 -Absorbed Energy vs Radial Deflection of wheel 7

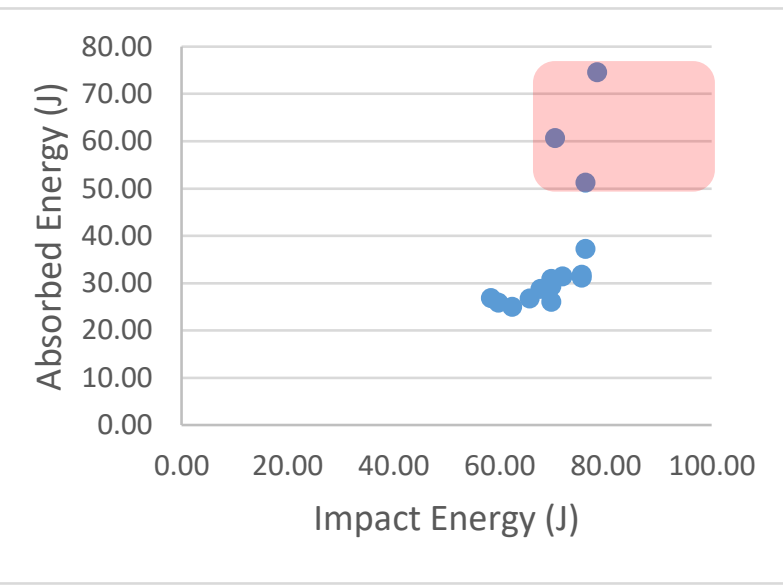

Figure 53 - Impact energy vs Absorbed energy of wheel 7

\subsubsection{Wheel 8}

Wheel 8, was a 20 spoke hole built with low spoke tension and impact loaded in line with the spoke. Wheel 8 also had a high energy threshold of $73 J$ (Figure 54) and experienced a radial displacement $8.3 \mathrm{~mm}$ before cracking (Figure 55). 


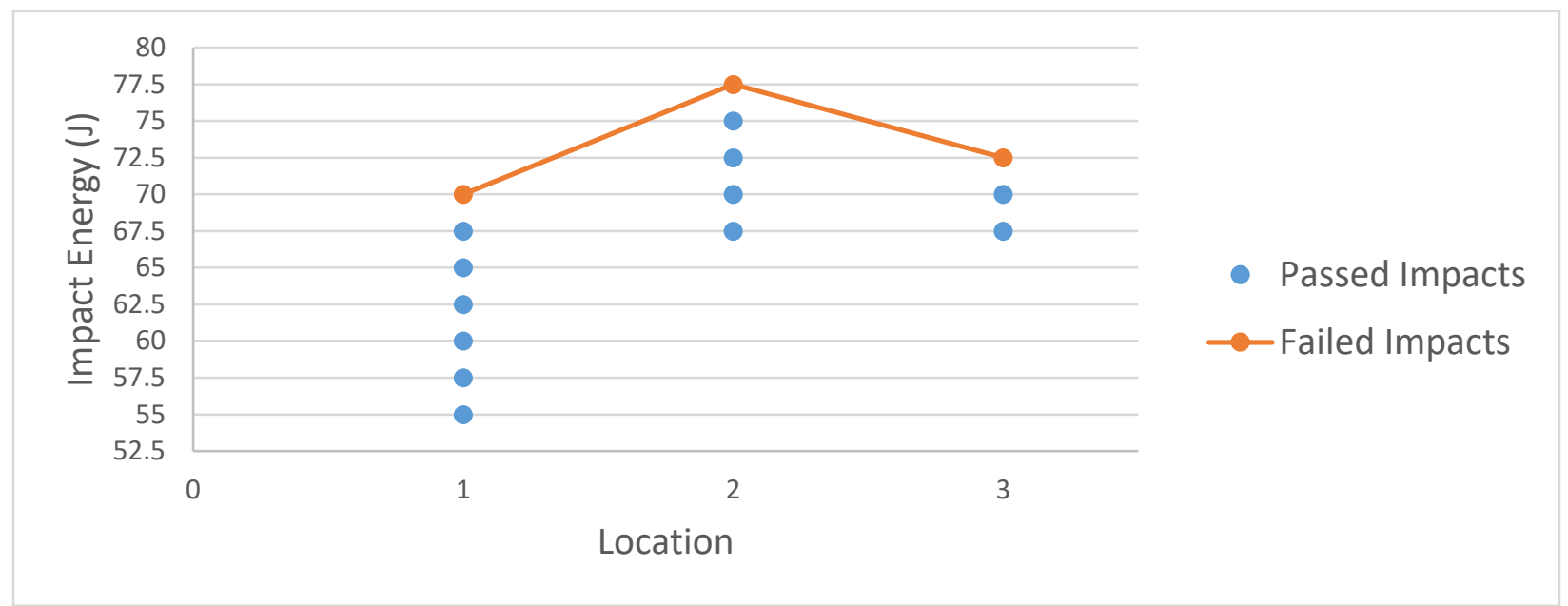

Figure 54 - Impact results of wheel 8 - 20 spoke wheel - low tension - in line

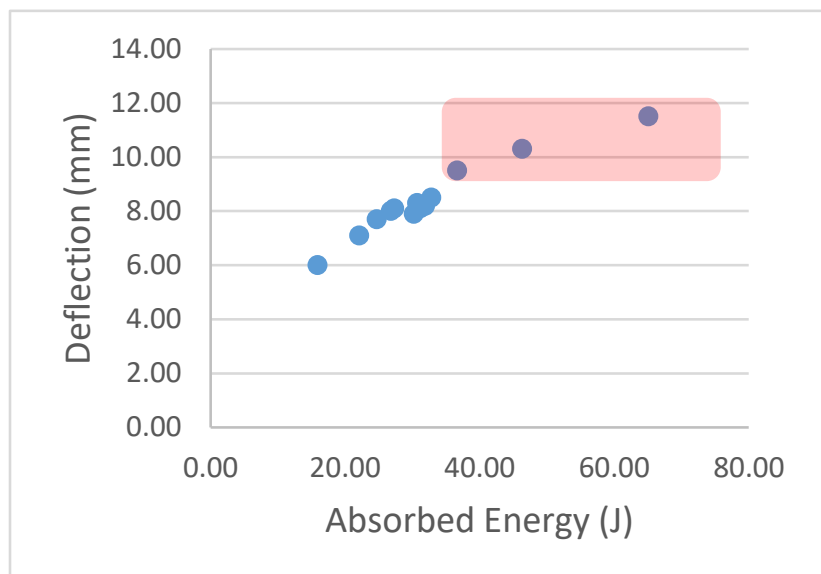

Figure 55 - Absorbed Energy vs Radial Deflection of wheel 8

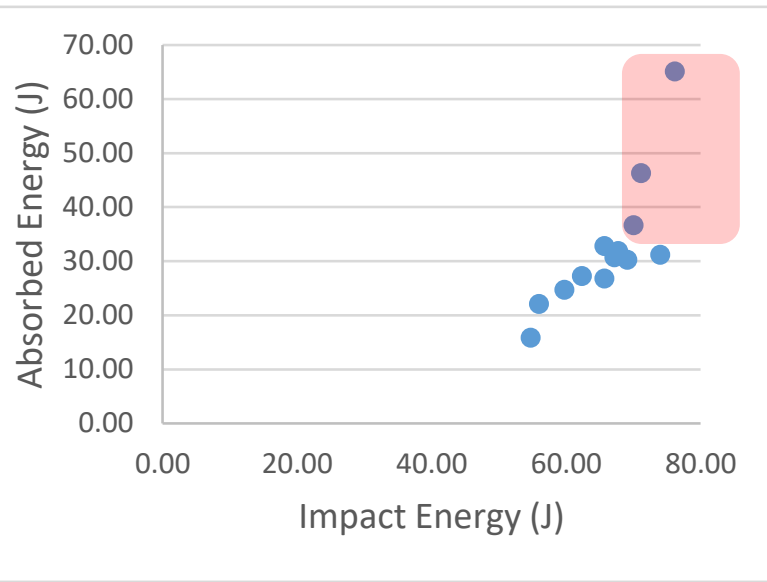

Figure 56 - Impact energy vs Absorbed energy of wheel 8

\subsection{Impact Test Summary}

The summary of the impact test results of all wheels is shown in Figures $57 \& 58$. The test showed that this wheel can take between 57.5-76 $J$ of impact depending on its build specification and the impact location. Generally, if the wheel is hit in between spokes, then a $60 \mathrm{~J}$ impact will result in a crack. Spoke tension and number of spokes did not affect the failure threshold when a wheel is impact loaded in between the spokes in this study. When a wheel is impacted in line with a spoke, it has shown to be much stronger. The 20 spoke wheel has a stronger threshold than the 24 spoke wheel. It was also observed that the 24 spoke wheels did not displace as much as the 20 spoke wheels. While the addition of spokes adds stiffness to the wheel, it concentrates more strain energy 
into the rim. The strain energy may exceed the failure threshold of the composite resulting in a crack. Spoke tension also had a direct correlation with failure threshold. Across the high-low spoke tension pairs, high spoke tension wheels experienced higher thresholds in 3 of the 4 wheels.

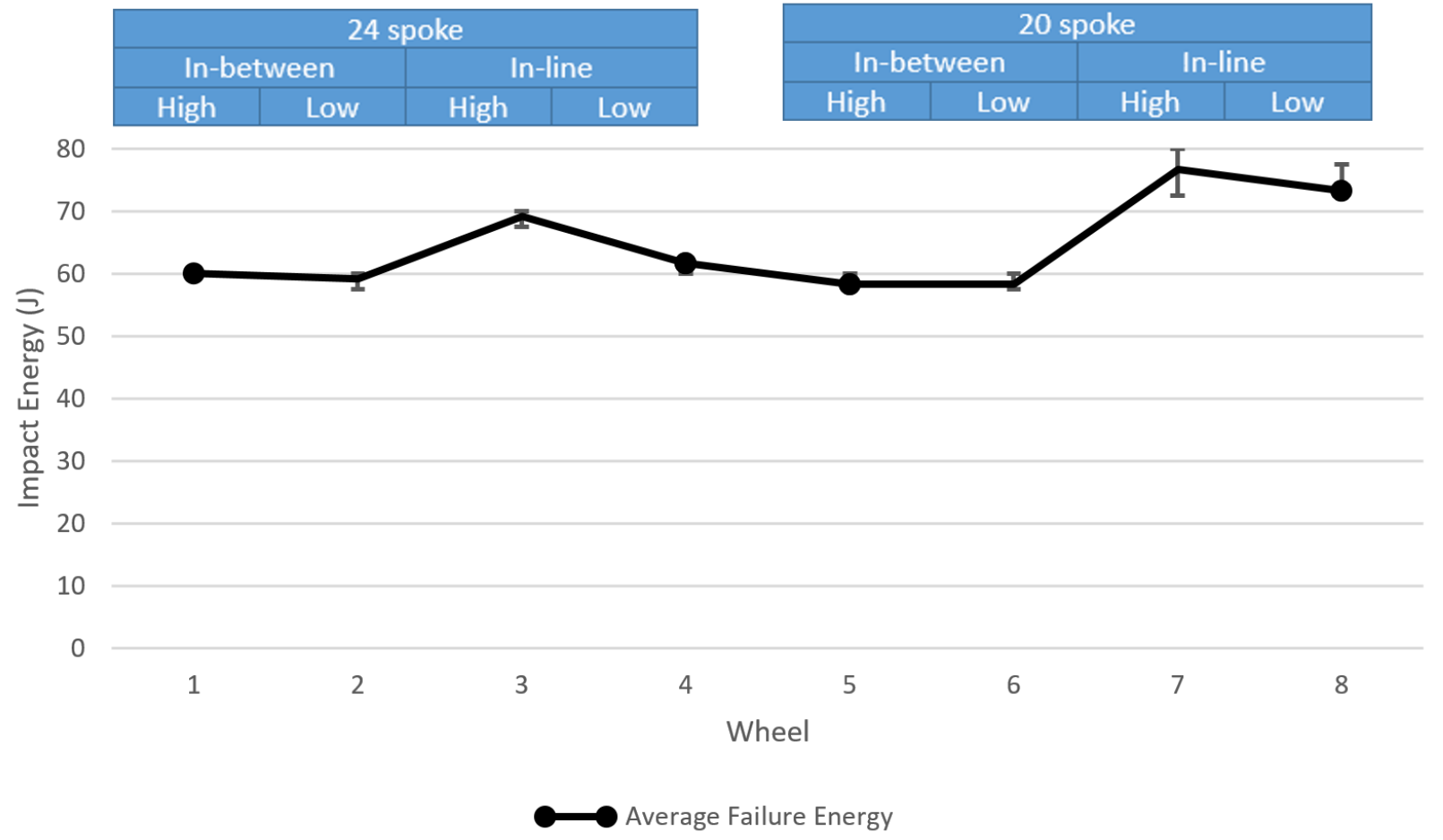

Figure 57 - Impact test summary of all wheels

The maximum radial displacement the rim could compress was $8.1 \mathrm{~mm}$ when loaded in between spokes and $8.8 \mathrm{~mm}$ when loaded in line with the spokes as can be seen in Figure 59. During impact, the spokes are buckling, however without instrumentation it is not possible to locate the moment the spoke is out of pretension and starts to buckle. Once the rim reaches these displacements, strain energy is concentrated within the rim and is released through fracture. 


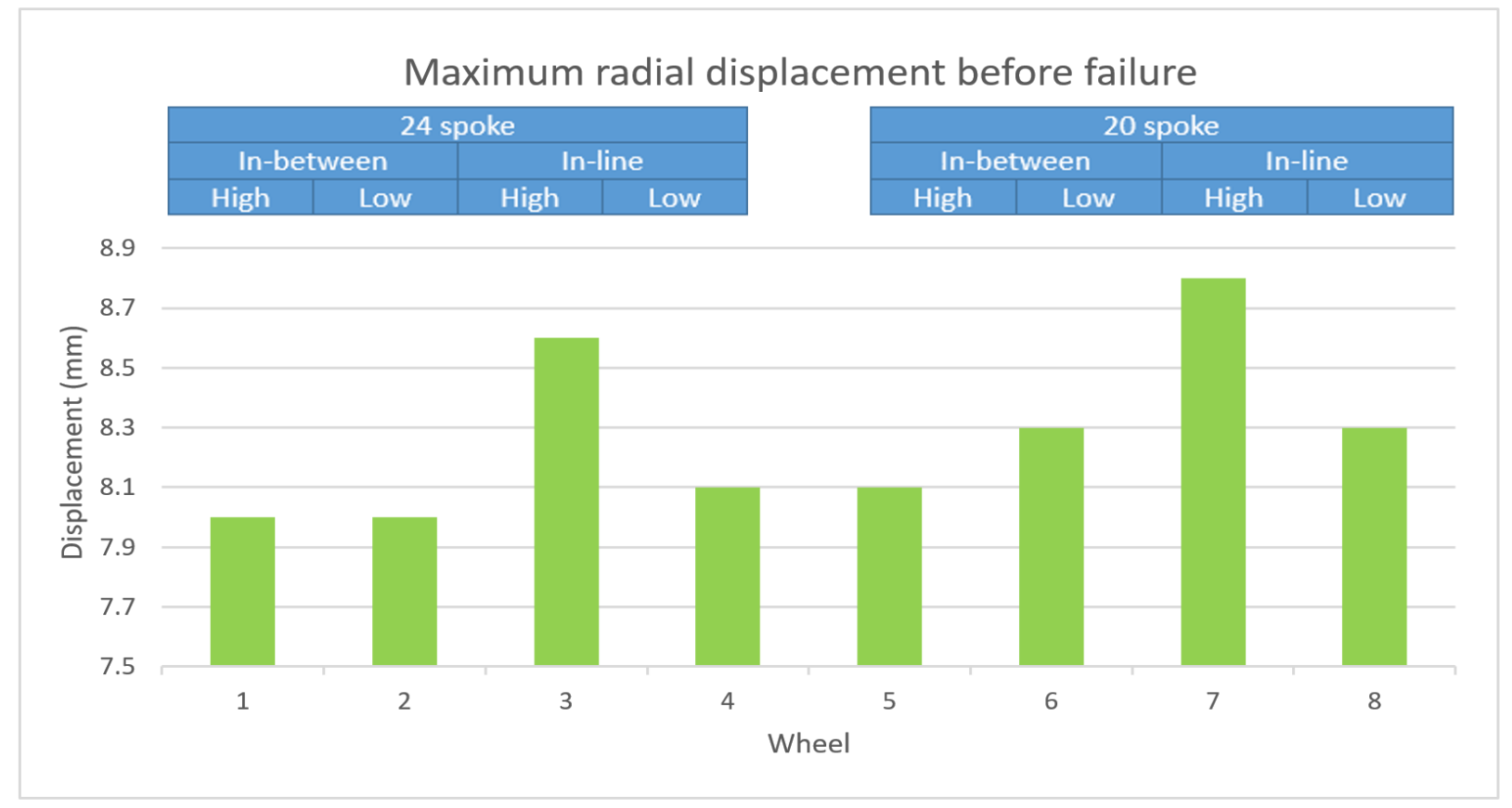

Figure 58 - Maximum rim radial displacement before failure

Another parameter analyzed was the energy absorbed per trial. It can be seen from Figure 59, that when the wheel absorbed more than $32 \mathrm{~J}$, a crack had initiated. This was across all trials performed in the 8 wheels. This shows that the structure is very brittle. As soon as the threshold has been crossed, a failure is imminent.

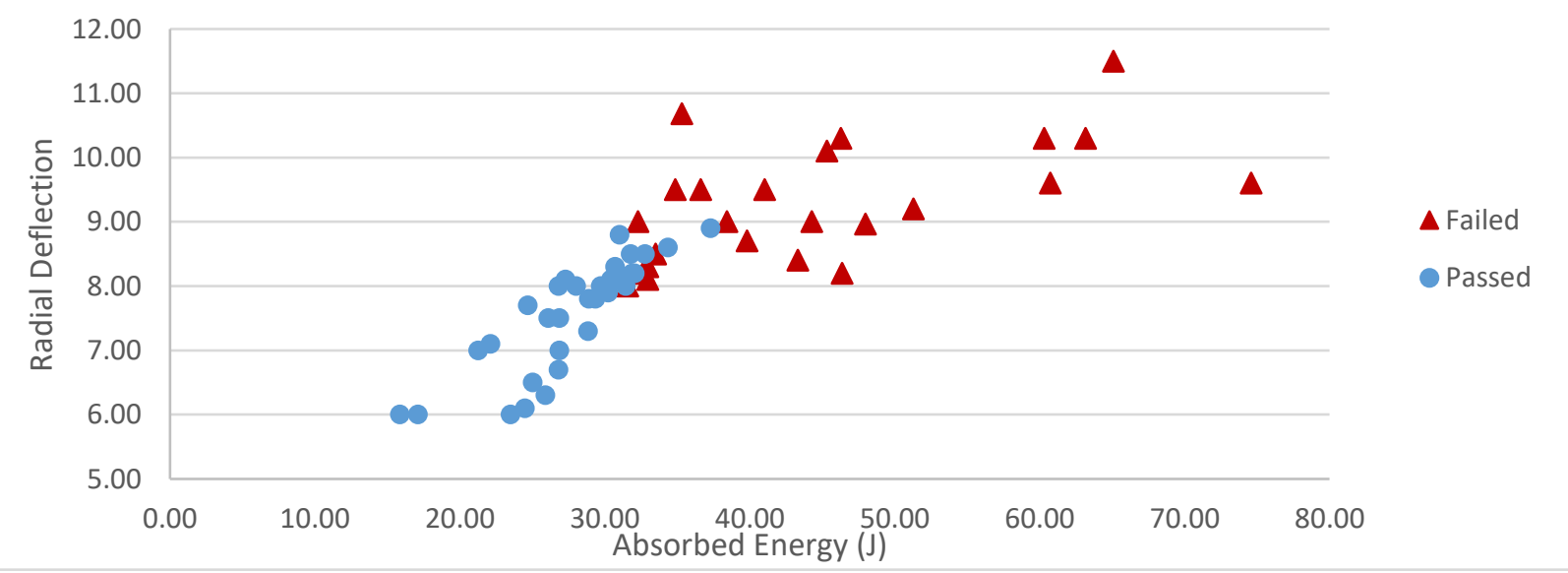

Figure 59 - Absorbed Energy vs Radial Deflection 


\subsection{Impact Damage Assessment (Microscopy)}

The next step was to investigate the fractures through the thermal and visual inspection data that had been collected. To categorize the cracks, they were measured with respect to a cylindrical coordinate system centered in the middle of the wheel. The lateral cracks were located on the top of the rim, where there was contact between the impactor and the rim. The radial cracks are orientated vertically running on the sidewalls of the rim $\left(90^{\circ}\right.$ ply orientation), and the tangential cracks are oriented along the sidewalls of the rim $\left(0^{\circ}\right.$ ply orientation). The crack lengths were measured across both sides of the wheel for each location and averaged. The data was then compared to the average energy absorbed among the failed trials in each test. By comparing energy absorbed and crack growth, it is possible to see how the structure reacts to increasing load with different configurations.

The following series of Figures 60-65 show the resulting damage of the wheel with increasing amounts of absorbed energy. Visual and thermographic inspection shows that lateral crack initiates from the top spoke installation holes, propagate to the edge of the rim, continue radially down the sidewall, and at R4/L4 on the rim wall split longitudinally.
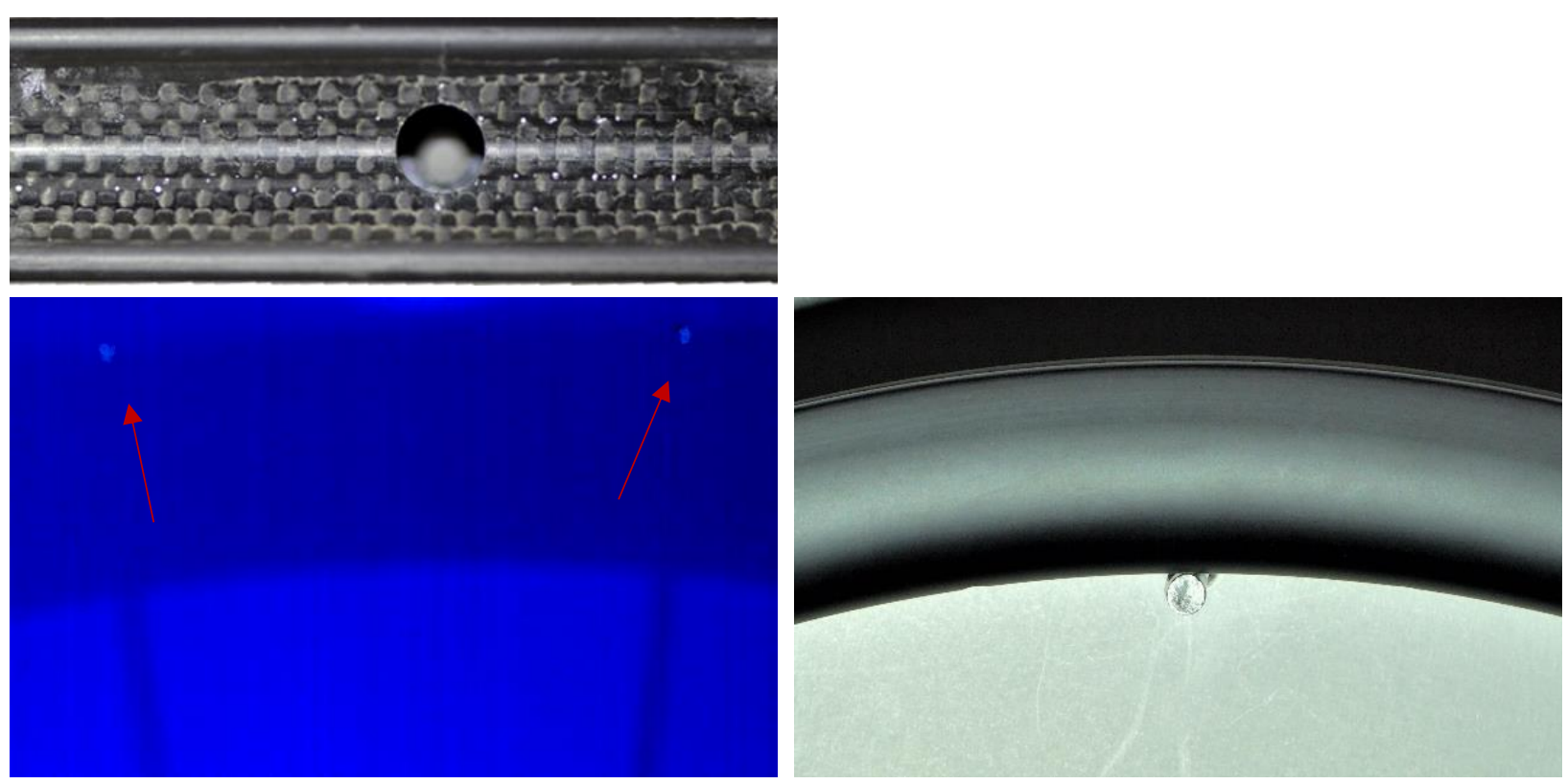

Figure 60 - Wheel 1 Location 1 - 33 J absorbed energy (a) top view - spoke hole (b) IR side view (c) side view 

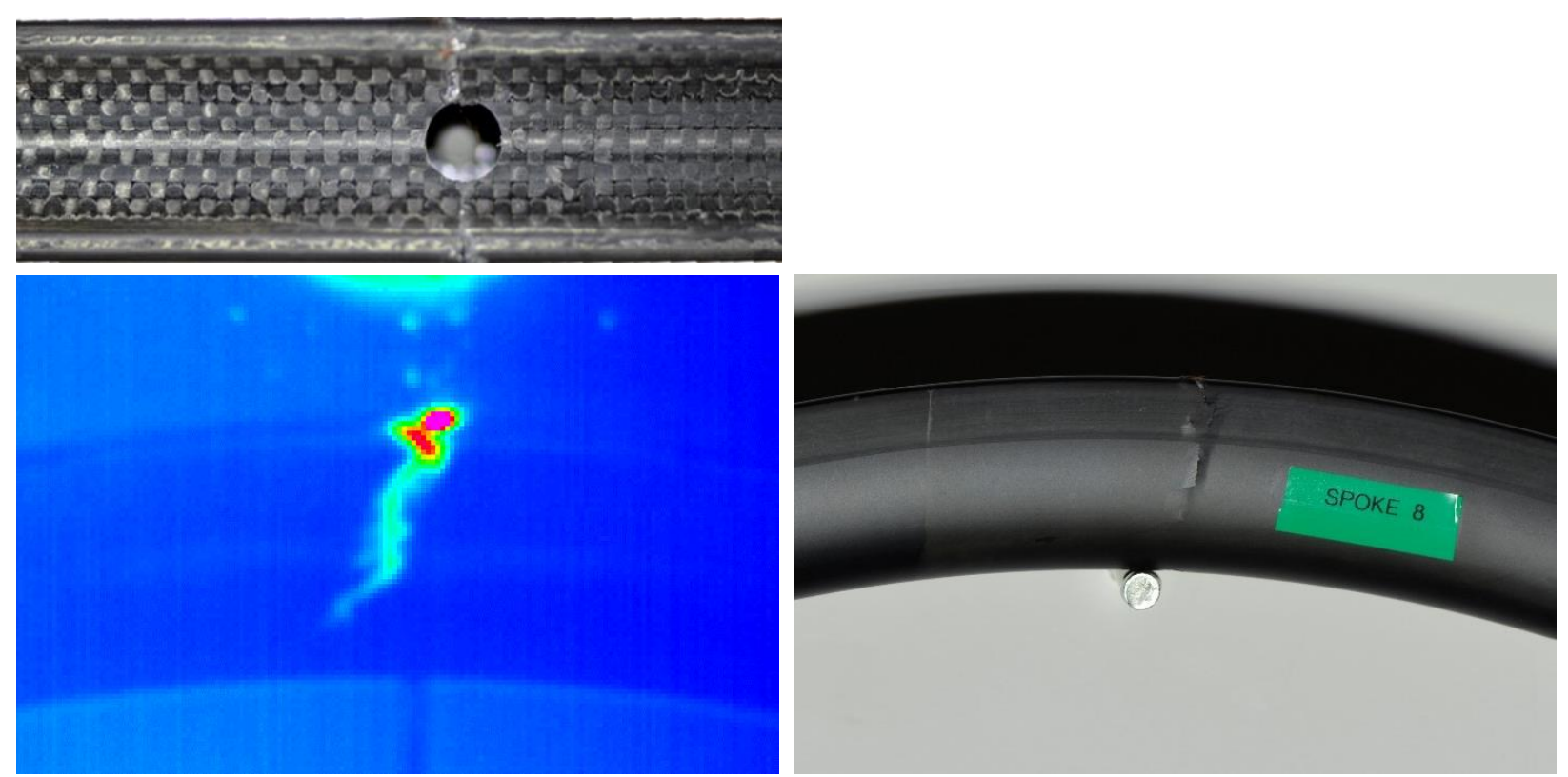

Figure 61 - Wheel 4 location 2 - $41 J$ absorbed energy (a) top view - spoke hole (b) IR side view (c) side view
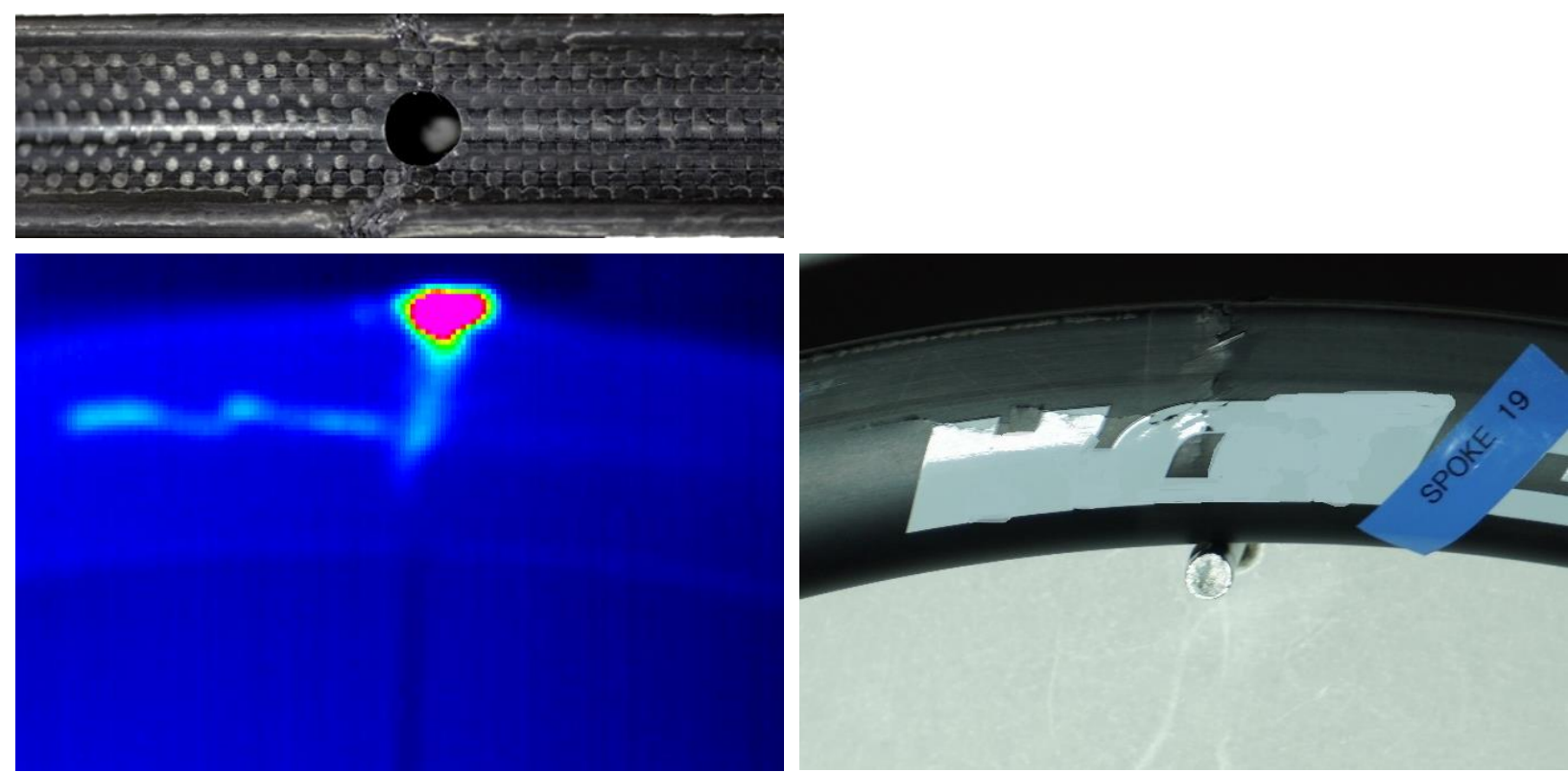

Figure 62 - Wheel 8 location 3 - $46 J$ absorbed energy (a) top view - spoke hole (b) IR side view (c) side view 

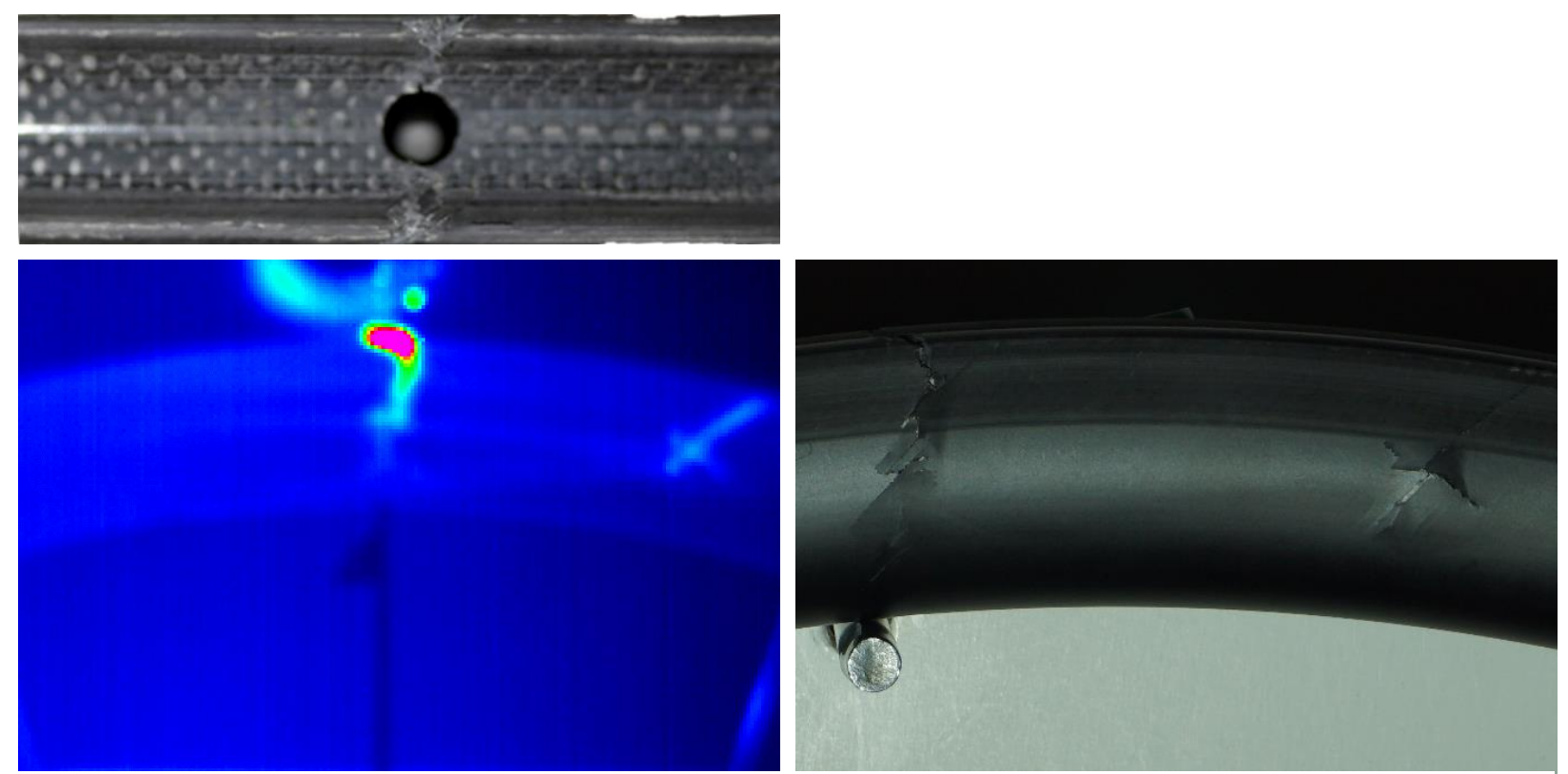

Figure 63 - Wheel 7 location 1 - $51 J$ absorbed energy(a) top view - spoke hole (b) IR side view (c) side view
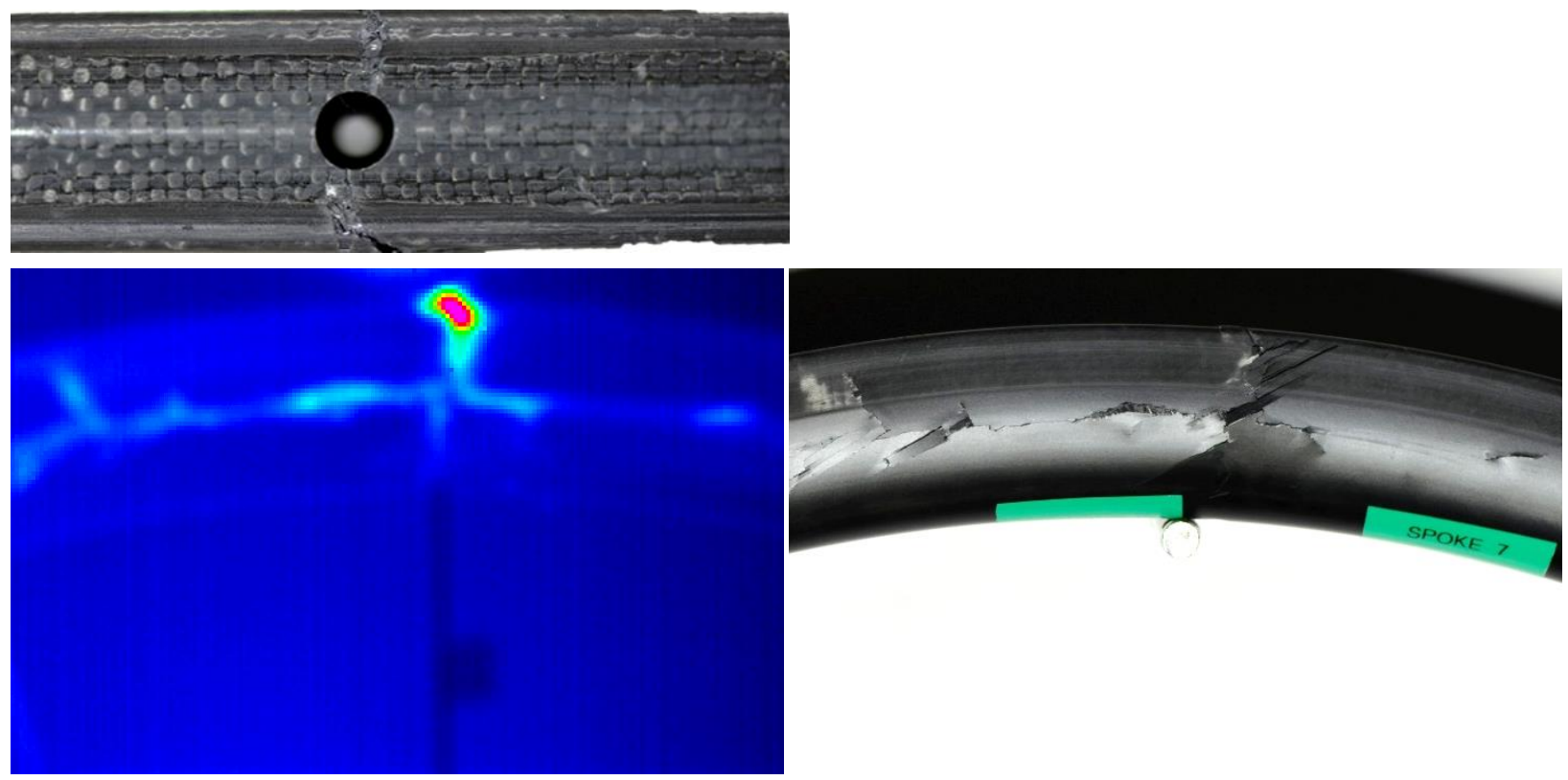

Figure 64 - Wheel 7 location 2 - $61 J$ absorbed energy (a) top view - spoke hole (b) IR side view (c) side view 

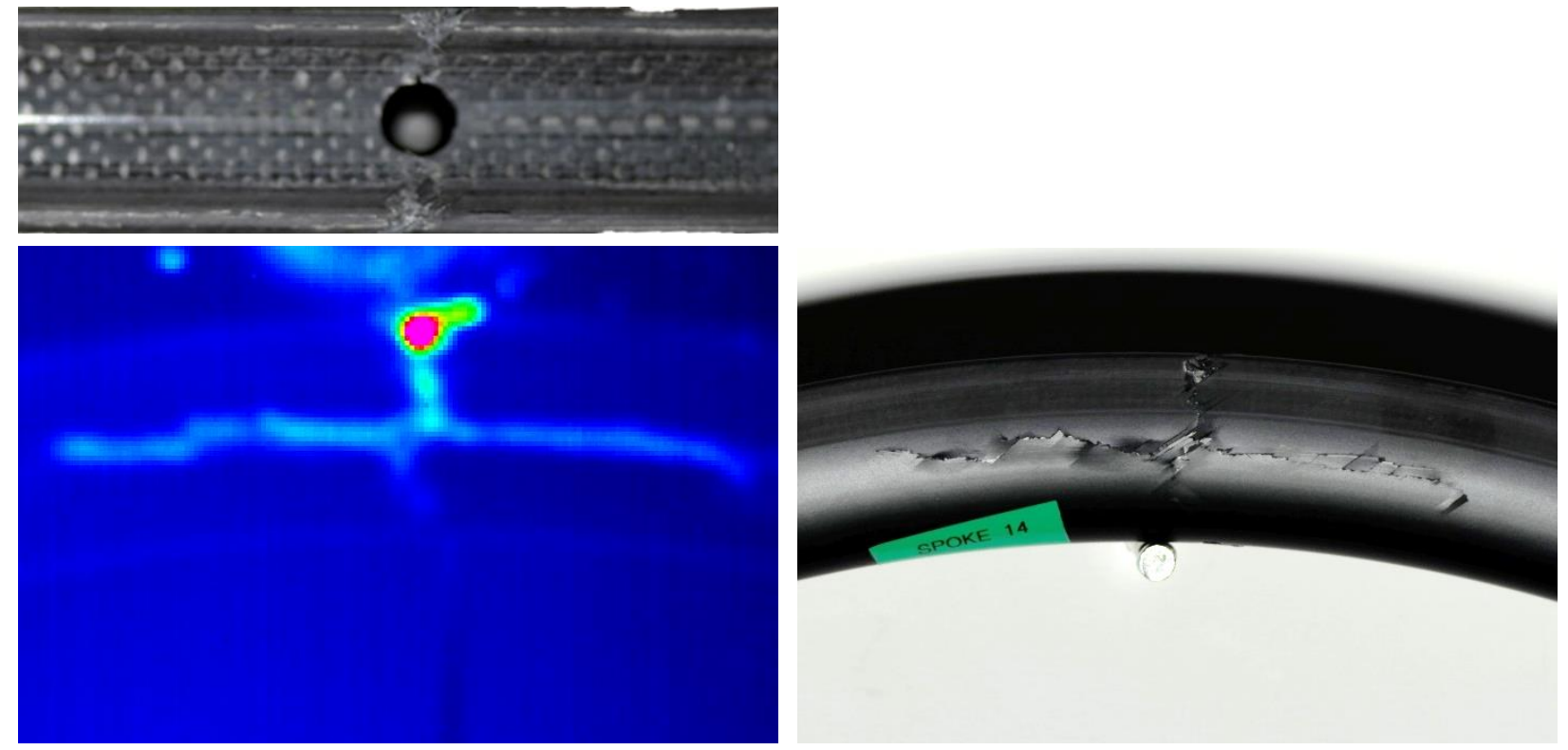

\section{Figure 65 - Wheel 7 location $374 J$ absorbed energy (a) top view - spoke hole (b) IR side view (c) side view}

The hot spots seen in Figures 61b-65b illustrate a release in energy which matches the visual crack shown in Figures 61c-65c. The thermal cracks in Figures $61 \mathrm{~b}-65 \mathrm{~b}$ are slightly wider than the actual cracks (Figures 61c-65c). This shows that the IR inspection method can show the extent of damage in evaluating fracture to a larger degree.

The lateral cracks start from the spoke access hole from and grow to the corner of the rim. The maximum length of crack in the lateral direction is $8.75 \mathrm{~mm}$. The maximum radial crack can be $35 \mathrm{~mm}$ due to the height of the rim cross section. Figure 66 shows that test 8 had the highest crack length and on average absorbed $10 J$ less than wheel 7 and $5 J$ less than wheel 3 . Wheel 5 also cracked more than wheel 2 and 4 . The large variance in trend can be attributed to the inconsistency in manufacturing quality. As documented in section 4, many randomly occurring manufacturing defects were discovered throughout the wheel.

The 2 weakest configurations (wheel 1 and 5) are of high spoke tension and impact in between the spokes. Wheel 2 and 6 are both in between spoke impact locations and low spoke tension configurations. It shows that the impacts in between spokes could absorb the least amount of energy before cracking. The 2 strongest wheels are 3 and 7, both of which have higher spoke tension and in line with spoke impacts. 
By looking at the number of spokes in each configuration, the 20 spoke wheels (test 5-8) are stronger than their 24 spoke wheel pair. This can be attributed to the added stiffness of the extra spokes. As the spokes require greater loads to displace them, there is a stress concentration in the rim leading to earlier failure.

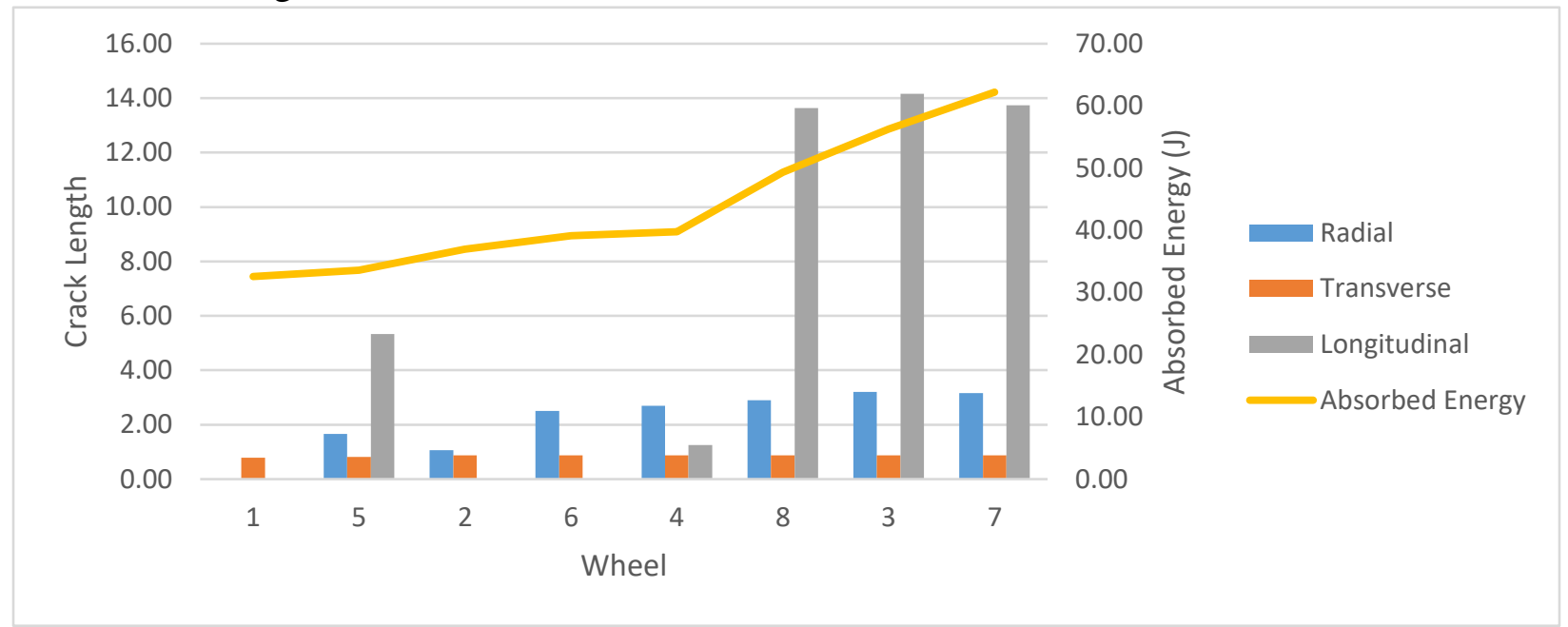

Figure 66 - Average crack length vs energy absorbed of each test

\subsection{Fracture initiation and growth}

In order to characterize the internal damage of the wheel, the tests were sectioned along the crack radially where the lateral and radial crack met. This allowed visual analysis on how the crack grew radially, laterally, and the longitudinally. The samples were set in LECOSET 7008 cold curing resin and polished down to 5microns to obtain a clear view of the cross section. High resolution photographs were taken using a Dino-Lite Pro desktop microscope. This process enabled the investigation of micro damage mechanisms such as delamination, fibre breakage, and cracking. Figure 67 shows the location of the sectioning cuts made on the wheels for crack analysis. 


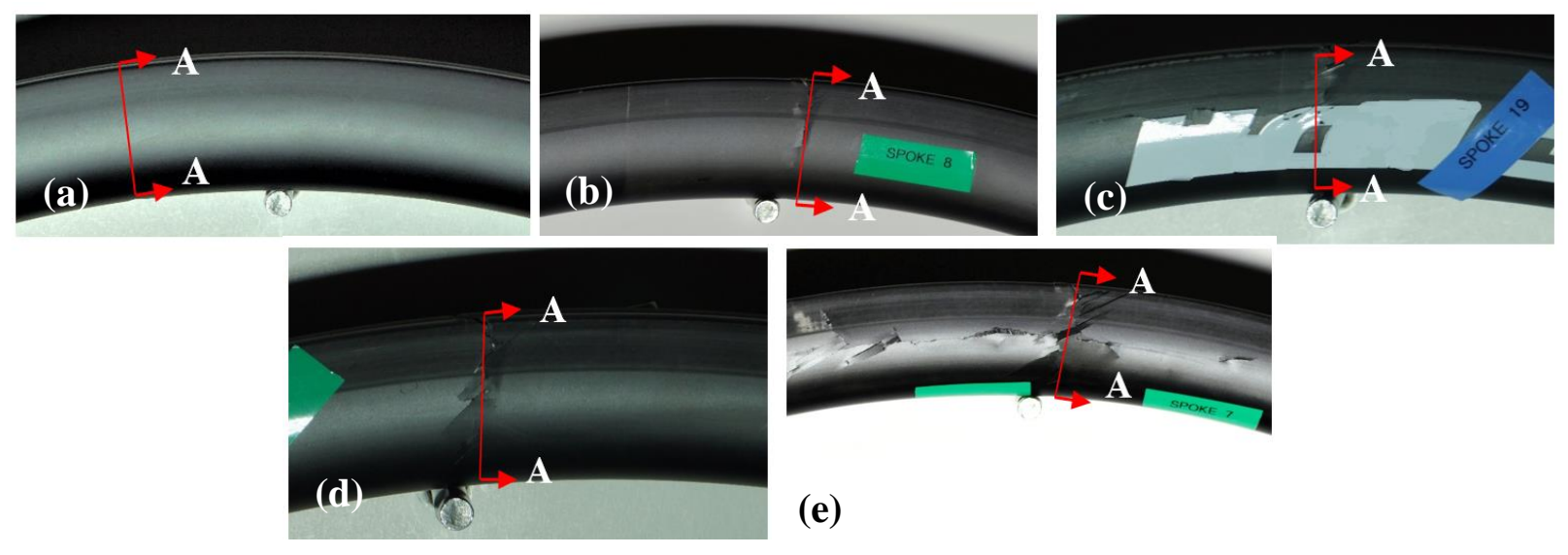

Figure 67 - Section cuts on (a) Wheel 1 (b) Wheel 4 (c) Wheel 8 (d) Wheel 7 location 1 (e) Wheel 7 location 2

Figure 68 highlights the fractures in wheel 1 location 1, where $33 J$ of energy was absorbed. Figure $68 \mathrm{~b}$ shows the ply ends at the spoke installation hole. Almost all the layers have separated, and the delamination continues to the corner of the rim cross section (Figure 68c/d). Upon impact, the top plies on the top section of the rim are under compression where the bottom plies are under tension. The clustered layers create a high bending mismatch which results in larger interlaminar stresses [44]. Due to the presence of the hole, there is a stress relief, and this allows the crack to initiate. The crack is $7 \mathrm{~mm}$ in length. At the corner, Figure 68d, micro cracks are found which show early signs of delamination in the bottom plies. The cracks do not travel around the rim into the side wall, however early signs of layer separation were found highlighted by Figures $68 \mathrm{e}$ and $68 \mathrm{f}$. This happens in R4 just after the resin rich ply tapered region in sections R1,R2,R3. Figure 68g shows a smooth curing of R5 with ply 20, however in Figure 68h the layers are clustered highlighting a manufacturing defect. In the bottom half of the rim cross section, there was no visible damage. 

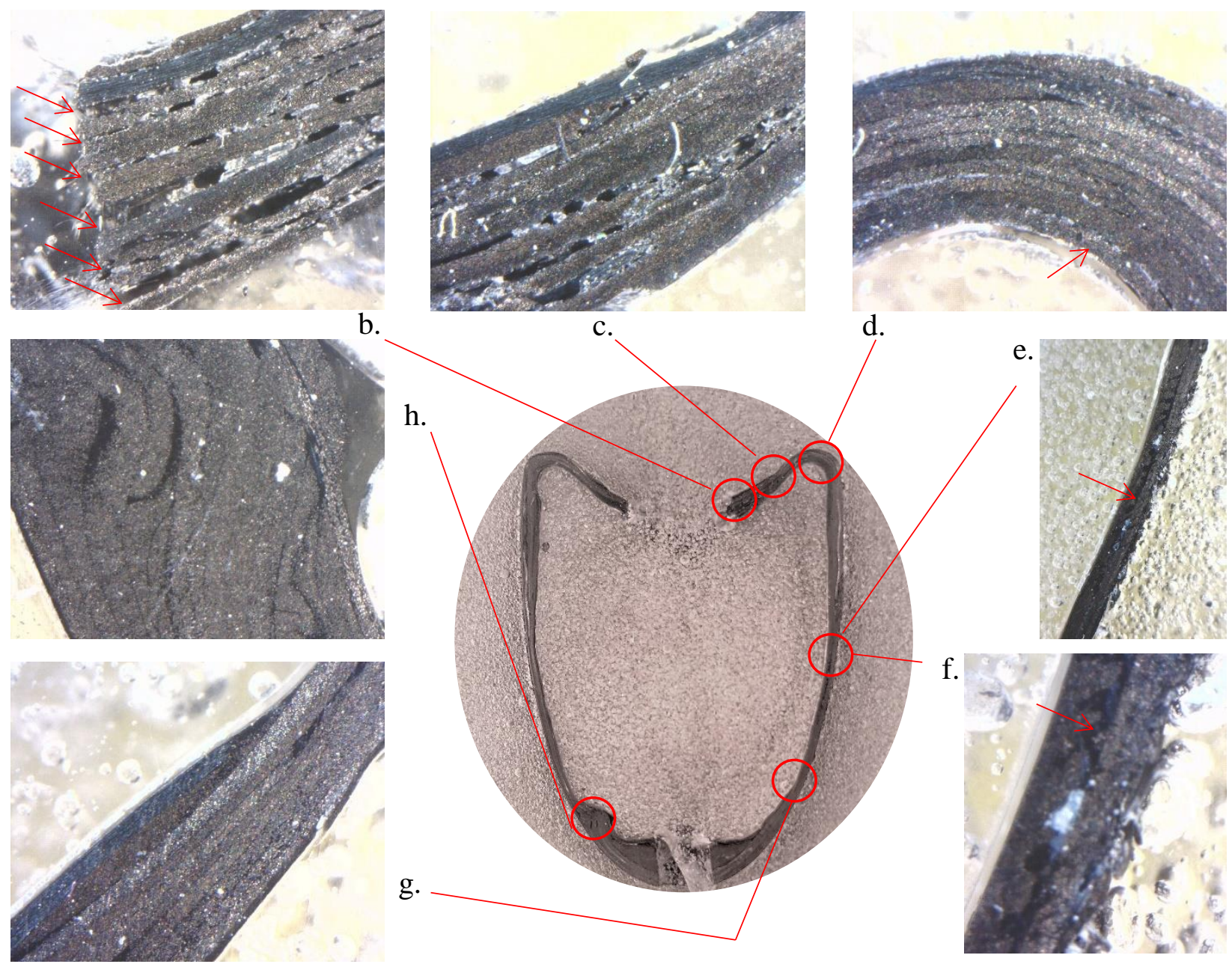

Figure 68 - Wheel 1 location 1 - $33 J$ absorbed - (a) cross section (b) layer separation at TM (b) layer separation at TR (c) microcracks and delamination TR1 (d) delamination inR1 (e)(f) delamination in L3/L4 (g) right cured zone R4/R5 (h) wavy fibers in L4/L5

In wheel 4 (Figure 69), $41 J$ of energy was absorbed which resulted in a full lateral crack and partial radial crack. In Figure 69c, a large amount of delamination can be seen between the bottom layers. The fracture than extends by breaking through layers 13 and 14 extends around the corner down the side walls (Figure 69d/e). Figure 69f/k show the L3/L4 and R3/R4 intersection, which is completely fractured. Figure $69 \mathrm{~g}$, shows how the plies have separated after the junction shown in Figure 69f. Figure 69h shows layer 20 separating from the spoke support region and Figure 69i shows the fiber fracture of layer 20 from left side. From these figures, it can be seen that the rim cross section behavior under impact is different in the top half then in the bottom. The intersection of L3/L4 and R3/R4, acts as a fixed point where the top half compresses. The outer plies along the sidewalls from L1/R1->L3/R3 are in tension while the inside plies are in compression. 

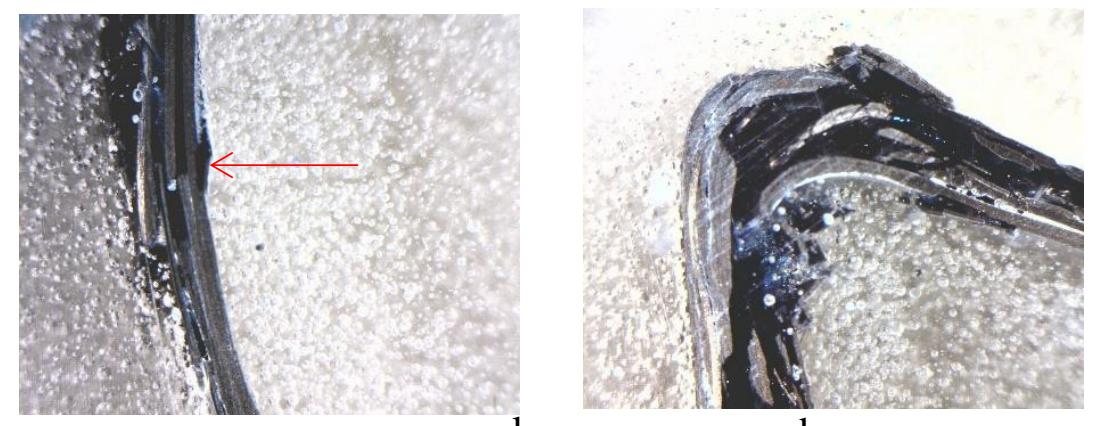

$\mathrm{k}$.

b.
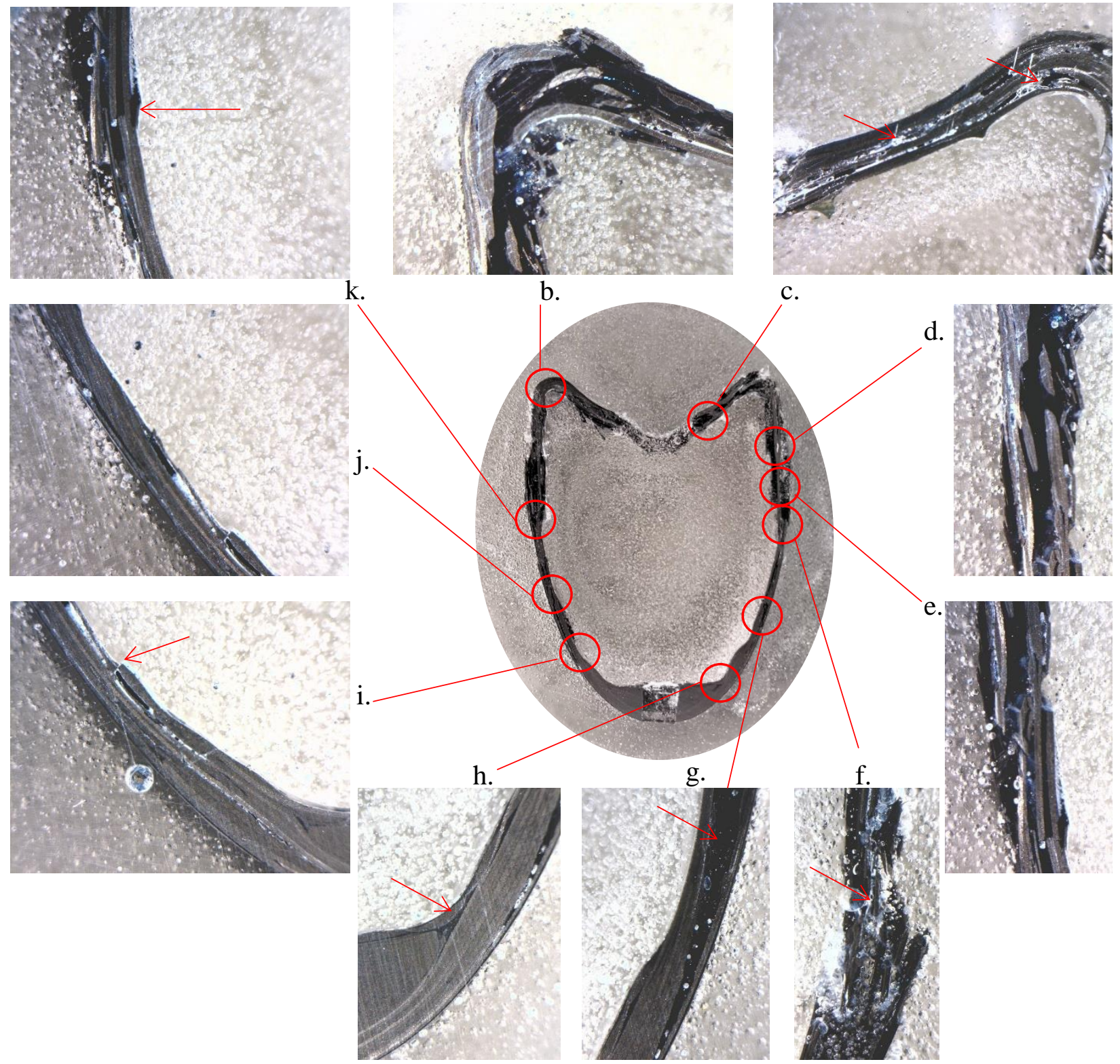

c.

Figure 69- Wheel $4-41 J$ absorbed energy - (a) cross section (b)fracture at L1 (c) delamination and layer separation in TM/TR/TR1 (d) delamination in R2 (e) delamination in $\mathrm{R3}$ (f) fiber fracture in R3/R4 (g) delamination in R4 (h) delamination in R5/BR (i) ply fracture in L4/L5 (j) ply frature in L4 (k)fiber fracture in L3/L4 
In wheel 8 location 2, (Figure 70) $46 J$ of energy was absorbed. In figure 70b/c, layer 17 has delamination, where layer 15 and 14 have fractured and separated. In Figure 70d, cracks have spread from the resin rich areas of R2/R3 and layer 2 has separated continuing into Figure 70e/f. In Figure 70f/g, layer 20 has fibre fracture from the R4/R5 and L4/L5 junction. The tangential crack is normal to the convergence point shown in Figure $70 \mathrm{~h}$.

In wheel 7 location 1 (Figure 71), $51 J$ of energy was absorbed. In comparison to wheel 4 (Figure 69), the additional energy compressed the rim further which can be seen by higher degree of ply separation in the left side wall (figure 71h). The convergence at L3/L4 is also apparent in this case and can be highlighted in Figure 71h. The right side had full fracture, where several plies had fragmented (Figure 71d). Larger tangential cracks were also observed in wheel 7 location 1, than in wheel 4 due to larger energy absorption (out-of-plane crack). There is also significantly higher delamination between layer 2 and 3 and this crack continues throughout the bottom section Figure $71 \mathrm{e} / \mathrm{f} / \mathrm{g}$.

In wheel 7 location 2 (Figure 72) $61 J$ of energy was absorbed. This resulted in cracks along the radial, lateral, and tangential directions. Compared to the $51 \mathrm{~J}$ case, larger and more destructive cracks were apparent. Figure 70c shows the crack start from the spoke installation hole. The delamination continues to the corner. In Figure 70d, the top layer has fractured and a very large separation between layer 1 and layer 2 can be seen. The separation continues into Figure 70e, and Figure 70f reconfirms the convergence point that was apparent in Figure 68-69. Below the convergence point, in Figure 70g, layer 20 shows separation which continues throughout its full length into Figure 70i, where it fractures. This ply fracture was also seen in Figure 69. In Figure 70h, ply 3 and 7 are also seen as separating, layer ply 1's separation increasing in Figures 70i and $70 \mathrm{j}$. In Figure $70 \mathrm{j}$, the middle plies stay neutral as continue through the convergence point. In reference to Figure 70a, the 2 convergence points (Figure 70f and 70j) are the least damaged areas in this profile however the tangential cracks were normal to this point. Figure 70k shows full fracture as layers 2,3,7,8,9 fragmented. Figure 70b mirrors Figure 70d with fibre breakage, separation and fracture. 

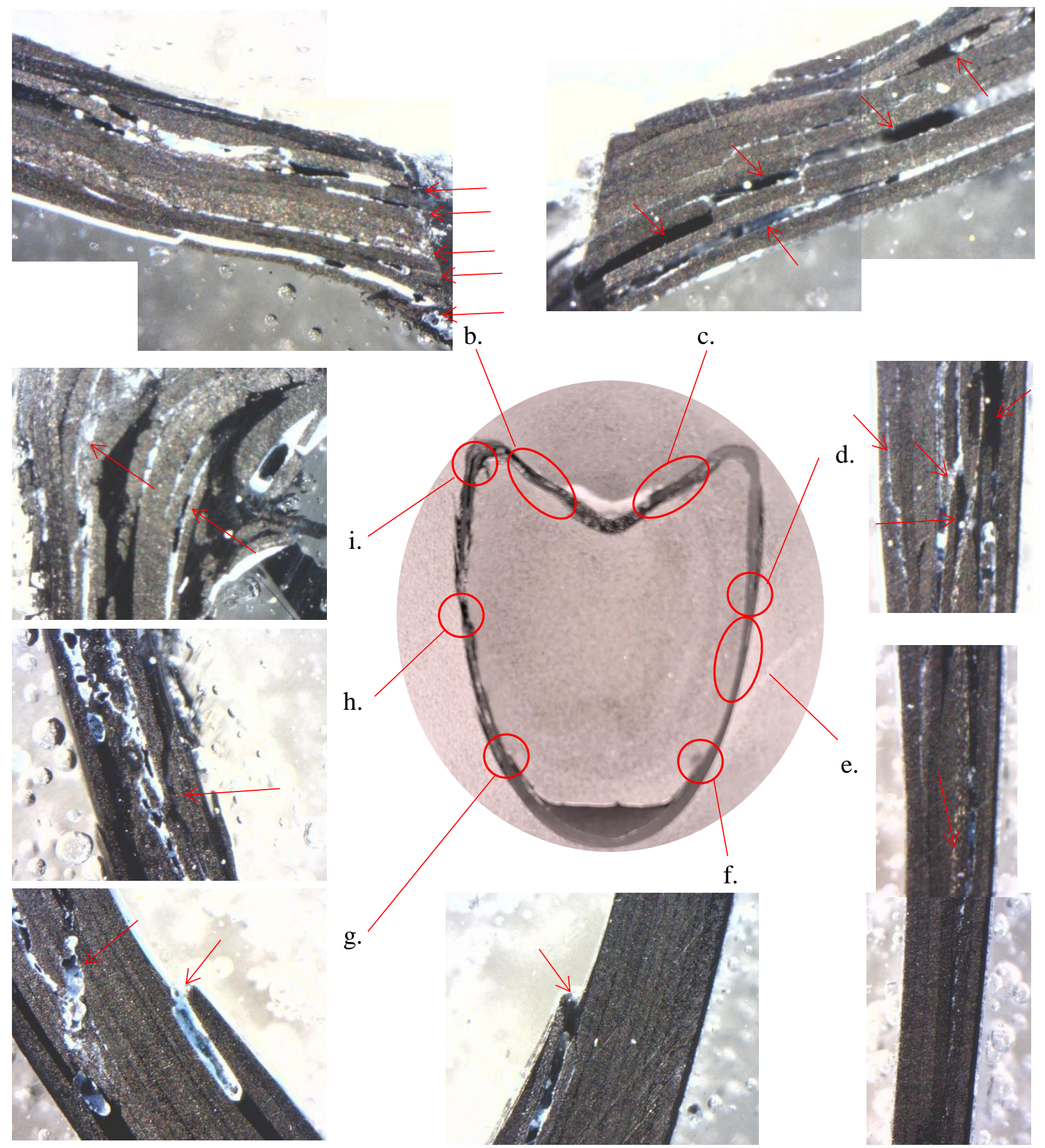

Figure 70 - Wheel 8 - $46 \mathrm{~J}$ absorbed energy - (a) cross section (b) delamination and

fracture in TL/TM (c) fiber fracture in TM/TR/TR1 (d) delamination and fracture in $R 3 / R 4$ (e) delamination in $R 4$ (f)fiber fracture in $R 4 / R 5$ (g) fiber fracture in $L 4 / L 5$ (h) fiber fracture in L3/L4 (i) delamination in R1 


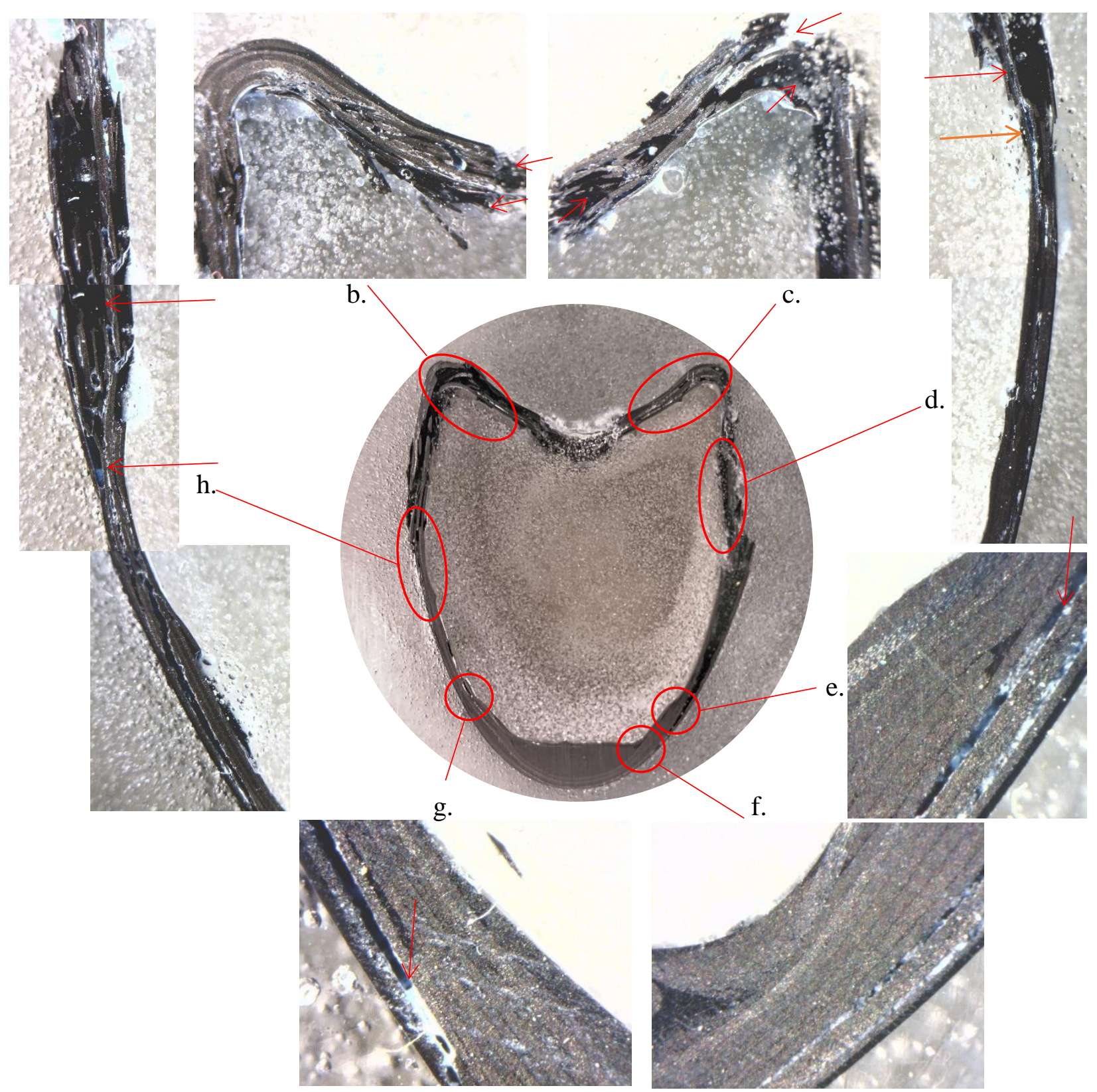

Figure 71 - Wheel 7 - location 1 - $51 J$ absorbed energy - (a) cross section (b) delamination across $\mathbf{L} 2 \rightarrow \mathrm{TM}$ (c) delamination across $\mathbf{T M} \rightarrow \mathbf{R 2}$ (d) delamination and fragmentation across $R 2 \rightarrow R 4$ (e) delamination in R5 (f) delamination in BR (g) delamination in BL (h) fiber fracture and delamination across $\mathrm{L} 2 \rightarrow \mathrm{L} 4$ 

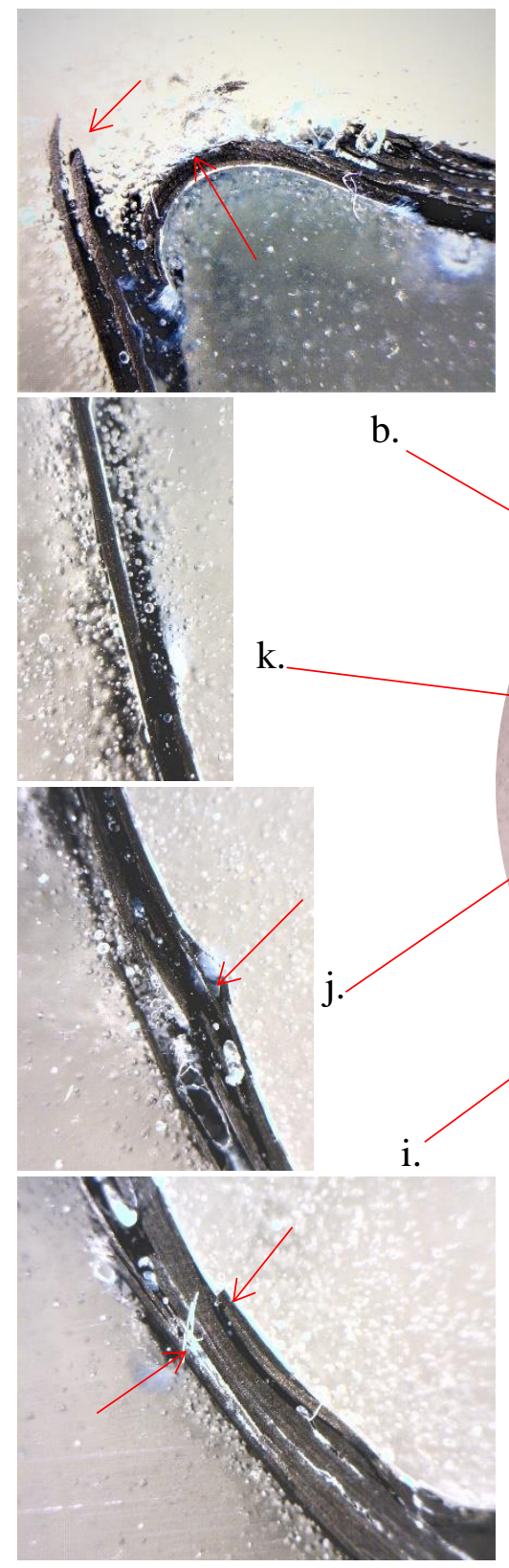

b.

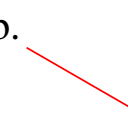

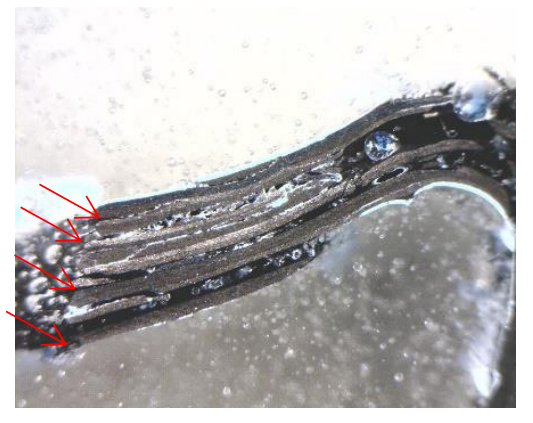

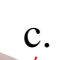

c.
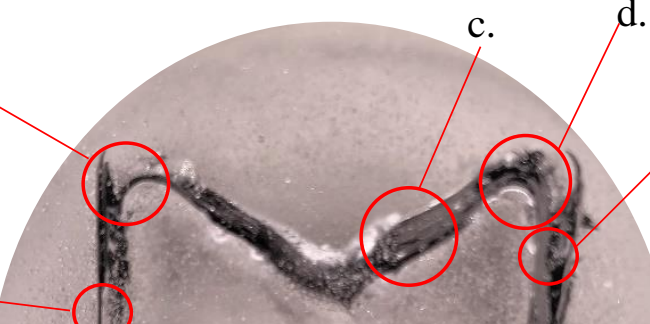

d.
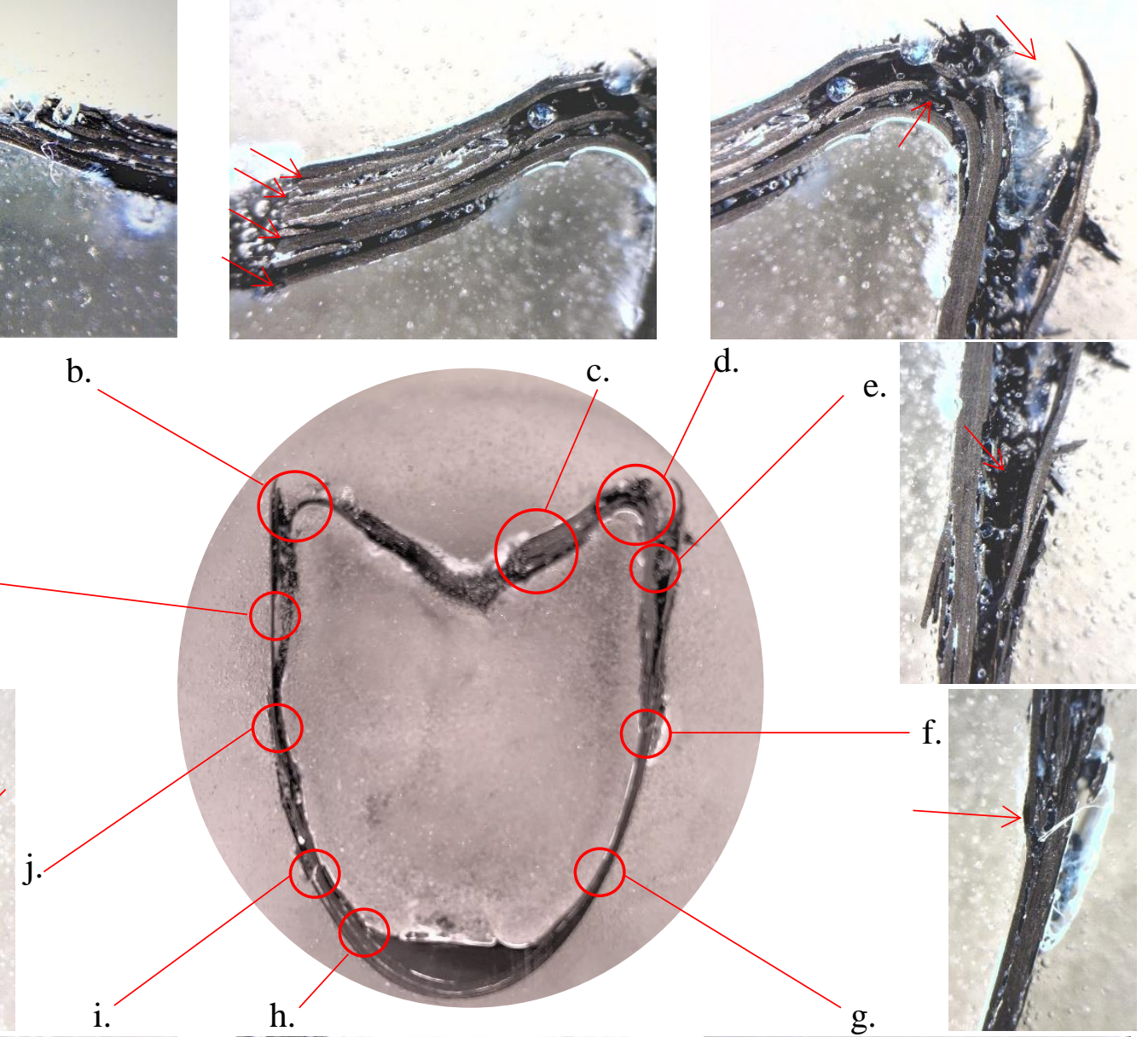

h.

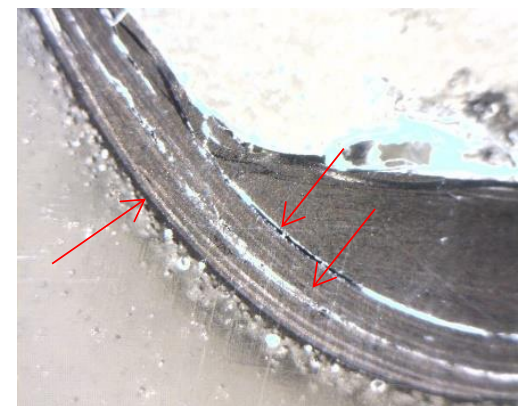

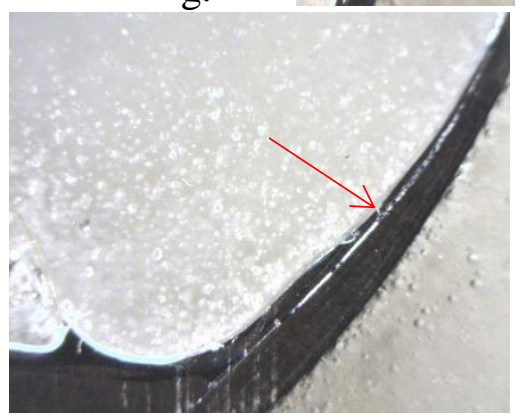

Figure 72 - (a) cross section (b) fracture and fragmentation across L2 $\rightarrow$ TM (c) delamination across $T M \rightarrow T R 1(d)$ delamination and fracture across $T R 1 \rightarrow R 2$ (e) delamination in $\mathrm{R} 2$ (f) fiber fracture in $\mathrm{R3} / \mathrm{R4}(\mathrm{g})$ delamination in $\mathrm{R5}$ (h) delamination in BL (i) delamination and fiber fracture in L4/L5 (j) delamination and fracture in L3/L4 (k) fracture and fragmentation in $\mathrm{R} 2 / \mathrm{R3}$ 


\subsection{Impact Response using IR thermography}

\subsubsection{PCT impact damage monitoring}

During impact, the kinetic energy from the impactor is transferred to the bicycle rim. Some of the energy is absorbed by the wheel structure and if it exceeds the strain energy threshold of the laminate, energy can be released in due to the formation of cracks, delamination and fibre breakage. During the formation of the new sub-structures, heat is released, and the temperature rise picked up by the IR camera. However, subsurface and microcracks can get lost in the noise of the sensor. In order to filter noise and ensure there were no microcracks after an impact had passed through visual inspection, a thermal inspection was performed before the load was incremented for the next test. The PCT post processing method was developed to check for the damage. An example of this sequence is shown in Figure 74 where the PCT images of the impacts on wheel 7 location 3 are shown. The wheel had passed impacts all impacts starting from $57.5 \mathrm{~J}$ to $75 \mathrm{~J}$ and failed at $77.5 \mathrm{~J}$.
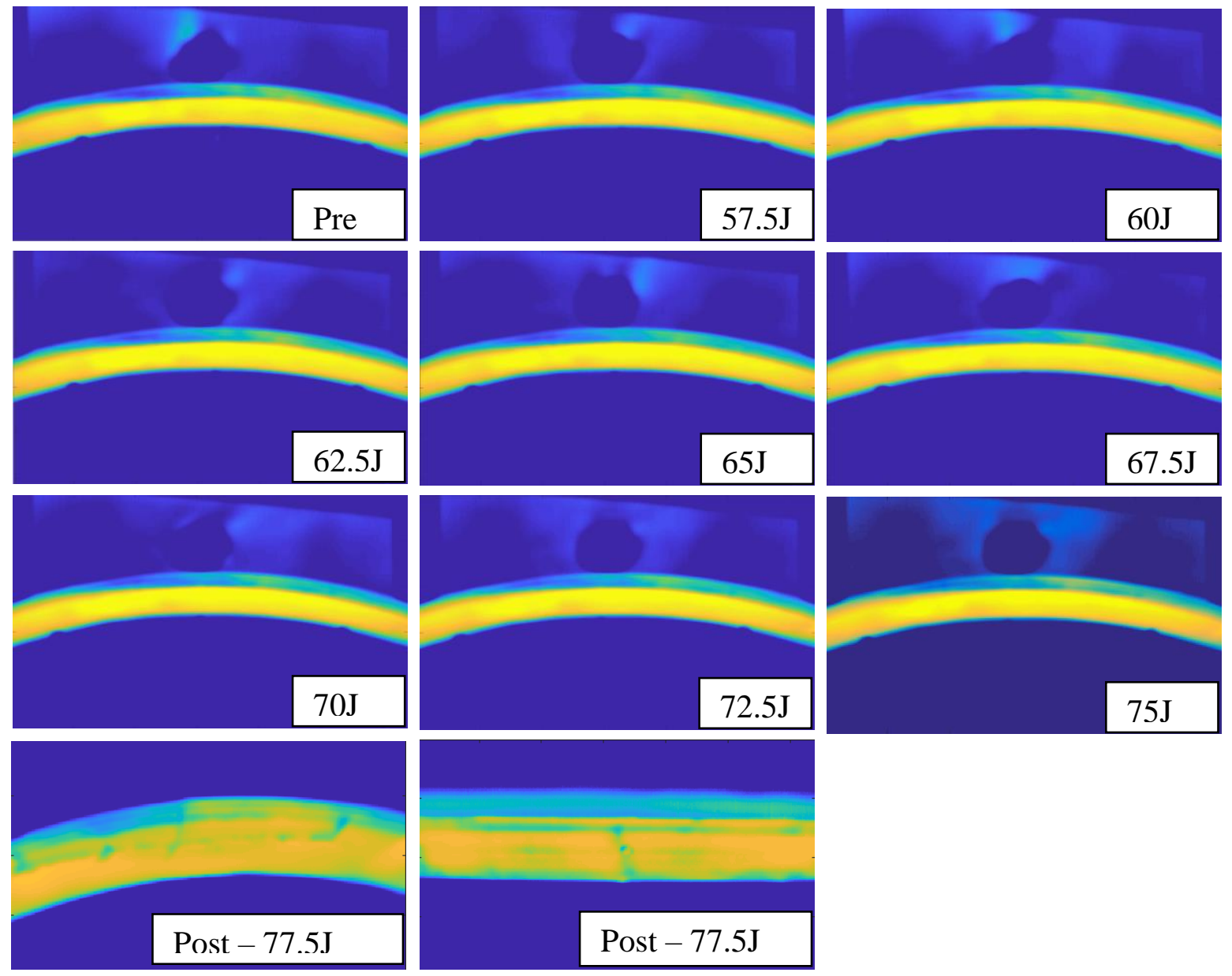

$75 \mathrm{~J}$

Figure 74 - Wheel 7 Location 3 PCT 


\subsubsection{IRT Impact Response}

Once a fracture has been initiated, the release in energy is displayed as an increase in temperature. Figures 75 shows the temperature profile of wheel 7 location 3 fractured at $77.5 \mathrm{~J}$ and absorbing $74 \mathrm{~J}$. During an impact, the material first experiences an elastic phase in which the energy is dissipated without plastic deformation and back into the impactor. This can be illustrated through the sudden surface temperature decrease. As the elastic threshold is reached, the energy absorbed in the plastic phase is dissipated across the surface and due to the brittle nature of composites, there is a sudden rise and the formation of fracture [24]. The fracture release causes a drop-in temperature due to the creation of new surfaces and is followed by a recovery phase which returns the surface temperature back to the ambient conditions.

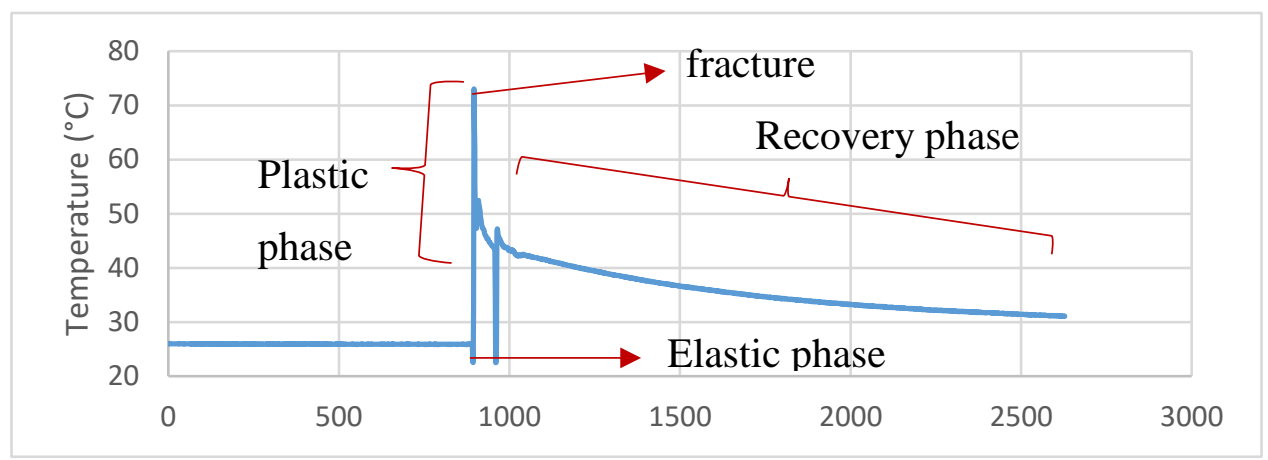

Figure 75 - Temperature profile of wheel 7 location 3 - 74J absorbed energy

In Figure 76, a linear relationship is seen between absorbed energy and change in the temperature. At the absorbed failure energy of 33J, there was a rise of only $0.8{ }^{\circ} \mathrm{C}$. At $41 \mathrm{~J}$, the temperature rose by $20^{\circ} \mathrm{C}$. Finally, at $74 \mathrm{~J}$ the temperature rose by $45^{\circ} \mathrm{C}$. With more testing, it might be possible to numerically model the heat release and absorbed energy by using the infrared thermography technique.

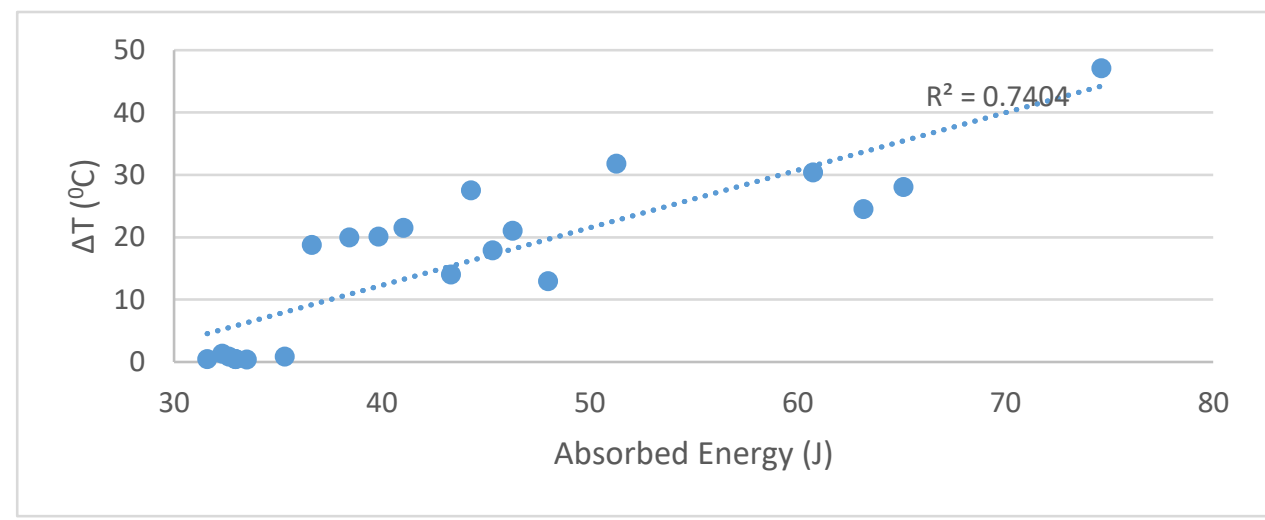

Figure 76 - Change in surface temperature from absorbed energy in failed specimens 


\subsection{Conclusion and Future Work}

Carbon fiber composites have become increasingly popular to manufacture high performance sports equipment due to their high strength-to weight ratios, environmental stability, and ability to create complex shapes. However, due to its infancy in the design of bicycle wheels, common field failures due to impact have risen. With the competitiveness of the cycling industry prioritizing light weighting as the feature of rapid innovation, there is a lack of data verifying the design choices and manufacturing quality of the retailed parts.

The main objective of this study was to describe the response of a bicycle wheel model that had experienced field failure through impact. The wheel had 2 models with spoke counts of 20 and 24. Spoke tension and impact location were other variables looked at in determining the impact response. The composite layup, stacking sequence and cross-sectional design for the 24 and 20 spoke wheels are the same.

The results revealed that this wheel had a damage initiation energy of $60 \mathrm{~J}$ when impacted in between spokes. When impacted in line with the spokes, damage initiation varied from $62.5 \mathrm{~J}$ to $80 J$ depending on the spoke count and spoke tension. Higher spoke tension resulted in a higher impact threshold, and the 20 spoke wheel build also showed a higher fracture energy threshold. Although the 24 spoke wheel is a stiffer system than the 20 spoke wheel, it resulted in more stress being concentrated in the rim and thus a lower impact failure threshold.

From all 8 cases, the maximum radial displacement the rim could compress before fracture was $8.8 \mathrm{~mm}$ and the minimum was $8 \mathrm{~mm}$. The 20 spoke wheel also showed that it was more flexible than the 24 spoke wheel due to its lower stiffness. When the spoke tension was high, the displacement was observed to be higher. This is because higher spoke tension required larger deflection before the spoke lost its pretension and transitioned into a buckling state. The rim does not crack unless the spokes directly underneath the load affected region have buckled.

Absorbed energy was calculated by the difference of the impact energy and the rebound energy of the weight. It was seen that anytime the wheel structure absorbed more than $32 \mathrm{~J}$ of energy, the initial failure modes had been activated.

Crack propagation was consistent among failures and became more evident by analyzing failures across different energy levels. At the initiation stages, the spoke installation hole provided a stress release and all cracks/delamination started from there. The crack would then grow laterally to the edge of the rim and fall radially. By looking at the design and stacking sequence, a transition 
area known as zone 3 and zone 4, which is the thinnest section of the rim sidewall acted as a convergence point. The top half the rim and the bottom of the rim both compressed about this point. This feature can be identified as a weakness in the structure. It was also observed that when the crack reaches the convergence point, the crack spreads out of plane and tangentially along the sidewall of the rim.

During the testing phases of this study, the IR PCT was a useful tool in monitoring the structural integrity of the rim and ensuring the wheel was devoid of cracks before being impacted again. Upon fracture, the temperature on the surface of the rim could increase by up to $40{ }^{\circ} \mathrm{C}$.

The results in the experiment have identified design and manufacturing considerations to improve the impact performance of this wheel model. It has successfully characterized the damage tolerance of the wheel, and the influence of wheel build parameters such as spoke tension, and number of spokes. It also identified the difference of fracture energy due to impact location. In order to improve this study, the following possibilities for future work can be proposed:

1. Low velocity testing with an instrumented jig consisting of load cells to measure impact force, spoke displacement, and energy absorbed by the rim and spokes. This will help to differentiate how much of the absorbed energy in this study was absorbed by not just the full system but by the rim and the spokes. Having this data, will be valuable in performing correlation analyses with numerical results and possibly modeling an elastic impact response zone of the wheel system.

2. Performing a full stacking sequence optimization to find the best layup for the load conditions without taking a weight penalty. Leveraging the current technology offered by FEA systems and improve the wheel characteristics.

3. Instead of performing high impact cases, perform compression after moderate impact cases to test long term durability which accurately represent road impacts from potholes and rough terrain.

4. Perform an explicit finite element model and include material/damage parameters to for accurate prediction of delamination and ply separation.

5. Create a database of testing for other wheel structures and designs to establish an industry baseline. 


\section{Appendix}

A1 Raw Data - Experimental Results

\begin{tabular}{|c|c|c|c|c|c|c|c|c|c|}
\hline No. & Wheel & Trial & $\begin{array}{c}\text { Spoke } \\
\text { Number }\end{array}$ & Einput & $\operatorname{Ei}(\mathrm{m} / \mathrm{s})$ & $\begin{array}{l}\text { Ereb } \\
(\mathrm{m} / \mathrm{s})\end{array}$ & $\mathrm{x}(\mathrm{mm})$ & $\begin{array}{l}\text { Post Spoke } \\
\text { Tension }\end{array}$ & Pass/Fail \\
\hline 1 & \multirow{6}{*}{1} & \multirow{2}{*}{$\mathrm{a}$} & $1->2$ & 57.5 & 3.05 & 2.42 & 7 & same & \\
\hline 2 & & & $1->2$ & 60 & 3.11 & 2.08 & 8.5 & same & $\mathrm{F}$ \\
\hline 3 & & \multirow{3}{*}{$\mathrm{b}$} & $9->12$ & 57.5 & & & & same & \\
\hline 4 & & & $9->12$ & 60 & 3.13 & 2.13 & 8.2 & same & \\
\hline 5 & & & $16->19$ & 57.5 & & & & same & $\mathrm{F}$ \\
\hline 6 & & $\mathrm{c}$ & $16->19$ & 60 & 3.15 & 2.20 & 8 & same & $\mathrm{F}$ \\
\hline 7 & \multirow{6}{*}{2} & \multirow[b]{2}{*}{$\mathrm{a}$} & $1->2$ & 55 & 2.99 & 2.08 & 7.8 & same & \\
\hline 8 & & & $1->2$ & 57.5 & 3.03 & 1.88 & $\begin{array}{c}10.682 \\
9\end{array}$ & same & $\mathrm{F}$ \\
\hline 9 & & \multirow{2}{*}{$\mathrm{b}$} & $10->11$ & 57.5 & & & & same & \\
\hline 10 & & & $10->11$ & 60 & 3.10 & 1.63 & 8.4 & same & $\mathrm{F}$ \\
\hline 11 & & \multirow{2}{*}{$\mathrm{c}$} & $17->18$ & 57.5 & & & & same & \\
\hline 12 & & & $17->18$ & 60 & 3.08 & 2.08 & 9 & same & $\mathrm{F}$ \\
\hline 13 & \multirow{8}{*}{3} & \multirow{3}{*}{$\mathrm{a}$} & 2 & 62.5 & 3.12 & 2.33 & 7.5 & same & \\
\hline 14 & & & 2 & 67.5 & 3.29 & 2.42 & 8.1 & same & \\
\hline 15 & & & 2 & 70 & 3.36 & 1.25 & 10.3 & less & $\mathrm{F}$ \\
\hline 16 & & \multirow{2}{*}{$\mathrm{b}$} & 10 & 65 & 3.25 & 2.33 & 8.2 & same & \\
\hline 17 & & & 10 & 67.5 & 3.29 & 1.88 & 10.1 & less & $\mathrm{F}$ \\
\hline 18 & & \multirow{3}{*}{$\mathrm{c}$} & 17 & 65 & 3.25 & 2.38 & 8.1 & same & \\
\hline 19 & & & 17 & 67.5 & 3.31 & 2.33 & 8.6 & same & \\
\hline 20 & & & 17 & 70 & 3.37 & 1.10 & 10.3 & less & $\mathrm{F}$ \\
\hline 21 & \multirow{5}{*}{4} & \multirow{2}{*}{$\mathrm{a}$} & 1 & 60 & 3.10 & 2.16 & 8.1 & same & \\
\hline 22 & & & 1 & 62.5 & 3.17 & 1.90 & 8.7 & same & $F$ \\
\hline 23 & & \multirow{2}{*}{$\mathrm{b}$} & 10 & 60 & 3.10 & 2.20 & 8 & same & \\
\hline 24 & & & 10 & 62.5 & 3.17 & 1.85 & 9.5 & same & $\mathrm{F}$ \\
\hline 25 & & $\mathrm{c}$ & 17 & 60 & 3.10 & 1.85 & 9 & same & $\mathrm{F}$ \\
\hline 26 & \multirow{6}{*}{5} & \multirow{2}{*}{$\mathrm{a}$} & $14->15$ & 55 & 3.00 & 2.25 & 6.1 & same & \\
\hline 27 & & & $14->15$ & 57.5 & 3.05 & 2.00 & 8.1 & same & $\mathrm{F}$ \\
\hline 28 & & \multirow{2}{*}{$\mathrm{b}$} & $1->20$ & 57.5 & 3.05 & 2.10 & 8.1 & same & \\
\hline 29 & & & $1->20$ & 60 & 3.10 & 2.00 & 9.5 & same & $\mathrm{F}$ \\
\hline 30 & & \multirow{2}{*}{$\mathrm{c}$} & $7->8$ & 55 & 2.95 & 2.21 & 6 & same & \\
\hline 31 & & & $7->8$ & 57.5 & 3.05 & 2.00 & 8.3 & same & $\mathrm{F}$ \\
\hline 32 & 6 & $\mathrm{a}$ & $1->2(6)$ & 60 & 3.10 & 1.37 & 8.963 & same & \\
\hline
\end{tabular}




\begin{tabular}{|c|c|c|c|c|c|c|c|c|c|}
\hline 33 & & & $1->2(6)$ & 57.5 & 3.03 & 2.04 & 8.8 & same & $\mathrm{F}$ \\
\hline 34 & & \multirow{3}{*}{$\mathrm{b}$} & $14->15$ & 55 & 3.00 & 2.50 & 6 & same & \\
\hline 35 & & & $14->15$ & 57.5 & 3.06 & 1.38 & 8.2 & same & \\
\hline 36 & & & $7->8$ & 55 & 2.95 & 2.04 & 8 & same & $\mathrm{F}$ \\
\hline 37 & & $\mathrm{c}$ & $7->8$ & 57.5 & 3.12 & 1.62 & 9 & same & $\mathrm{F}$ \\
\hline 38 & \multirow{15}{*}{7} & \multirow{8}{*}{$\mathrm{a}$} & 1 & 57.5 & 3.06 & 2.25 & 7 & same & \\
\hline 39 & & & 1 & 60 & 3.10 & 2.33 & 6.3 & same & \\
\hline 40 & & & 1 & 62.5 & 3.17 & 2.45 & 6.5 & same & \\
\hline 41 & & & 1 & 65 & 3.25 & 2.50 & 6.7 & same & \\
\hline 42 & & & 1 & 67.5 & 3.30 & 2.50 & 7.3 & same & \\
\hline 43 & & & 1 & 72.5 & 3.40 & 2.55 & 8 & same & \\
\hline 44 & & & 1 & 75 & 3.48 & 2.67 & 8.1 & same & \\
\hline 45 & & & 1 & 77.5 & 3.50 & 2.00 & 9.2 & less & $\mathrm{F}$ \\
\hline 46 & & \multirow{2}{*}{$\mathrm{b}$} & 7 & 70 & 3.35 & 2.65 & 7.5 & same & \\
\hline 47 & & & 7 & 72.5 & 3.37 & 1.25 & 9.6 & less & $\mathrm{F}$ \\
\hline 48 & & \multirow{5}{*}{$\mathrm{c}$} & 14 & 70 & 3.35 & 2.55 & 7.8 & same & \\
\hline 49 & & & 14 & 72.5 & 3.35 & 2.50 & 8.2 & same & \\
\hline 50 & & & 14 & 75 & 3.48 & 2.65 & 8.5 & same & \\
\hline 51 & & & 14 & 77.5 & 3.50 & 2.50 & 8.9 & same & \\
\hline 52 & & & 14 & 80 & 3.55 & 0.77 & 9.6 & less & $\mathrm{F}$ \\
\hline 53 & \multirow{13}{*}{8} & \multirow{3}{*}{$\mathrm{a}$} & 8 & 72.5 & 3.33 & 2.50 & 7.9 & same & \\
\hline 54 & & & 8 & 75 & 3.45 & 2.62 & 8.1 & same & \\
\hline 55 & & & 8 & 77.5 & 3.50 & 1.33 & 11.5 & less & $\mathrm{F}$ \\
\hline 56 & & \multirow{7}{*}{$\mathrm{b}$} & 14 & 55 & 2.97 & 2.50 & 6 & same & \\
\hline 57 & & & 14 & 57.5 & 3.00 & 2.33 & 7.1 & same & \\
\hline 58 & & & 14 & 60 & 3.10 & 2.38 & 7.7 & same & \\
\hline 59 & & & 14 & 62.5 & 3.17 & 2.37 & 8.1 & same & \\
\hline 60 & & & 14 & 65 & 3.25 & 2.30 & 8.5 & same & \\
\hline 61 & & & 14 & 67.5 & 3.29 & 2.42 & 8.3 & same & \\
\hline 62 & & & 14 & 70 & 3.36 & 2.32 & 9.5 & less & $\mathrm{F}$ \\
\hline 63 & & \multirow{3}{*}{$\mathrm{c}$} & 19 & 67.5 & 3.25 & 2.50 & 8 & same & \\
\hline 64 & & & 19 & 70 & 3.30 & 2.40 & 8.2 & same & \\
\hline 65 & & & 19 & 72.5 & 3.38 & 2.00 & 10.3 & less & $\mathrm{F}$ \\
\hline
\end{tabular}




\section{A2 PCT code}

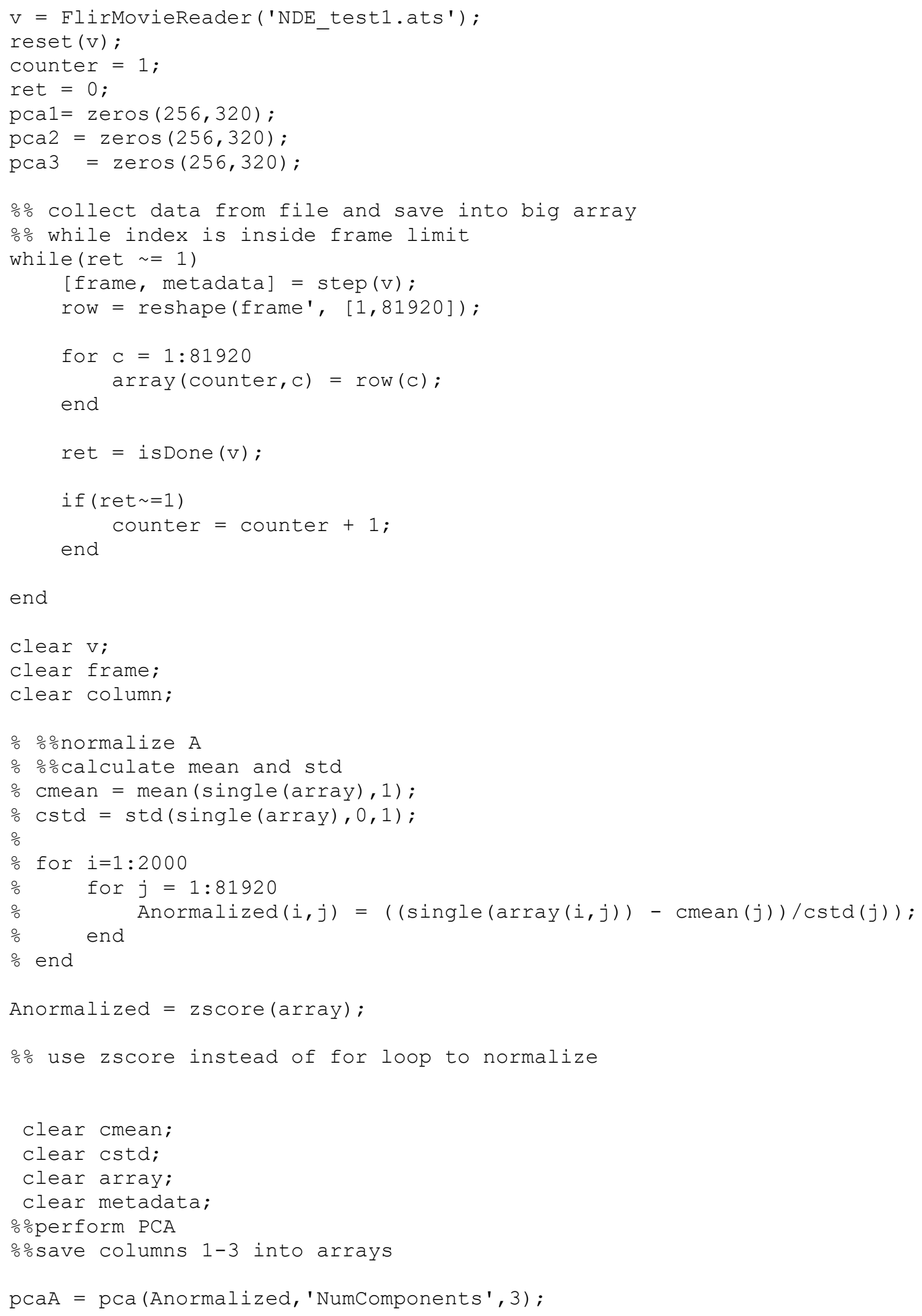




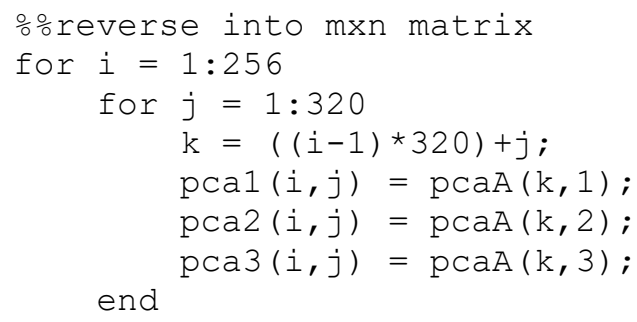

응lot

\section{A3 Unsymmetrical laminates Modulus formulae - CLT [35]}

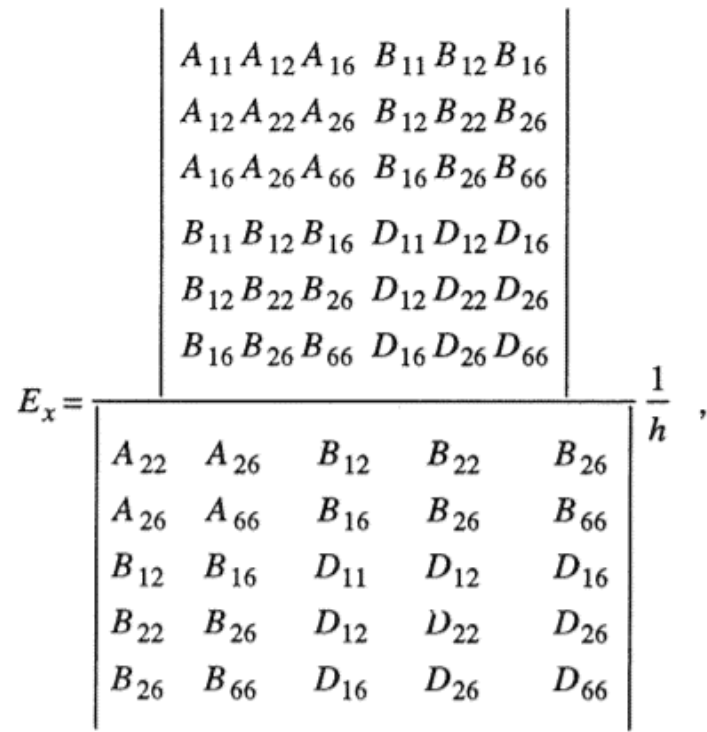

$$
\begin{aligned}
& E_{y}=\frac{\left|\begin{array}{lll}
A_{11} A_{12} A_{16} & B_{11} B_{12} B_{16} \\
A_{12} A_{22} A_{26} & B_{12} B_{22} B_{26} \\
A_{16} A_{26} A_{66} & B_{16} B_{26} B_{66} \\
B_{11} B_{12} B_{16} & D_{11} D_{12} D_{16} \\
B_{12} B_{22} B_{26} & D_{12} D_{22} D_{26} \\
B_{16} B_{26} B_{66} & D_{16} D_{26} D_{66}
\end{array}\right|}{\left|\begin{array}{lllll}
A_{11} & A_{16} & B_{11} & B_{12} & B_{16} \\
A_{16} & A_{66} & B_{16} & B_{26} & B_{66} \\
B_{11} & B_{16} & D_{11} & D_{12} & D_{16} \\
B_{12} & B_{26} & D_{12} & D_{22} & D_{26} \\
B_{16} & B_{66} & D_{16} & D_{26} & D_{66}
\end{array}\right|},
\end{aligned}
$$

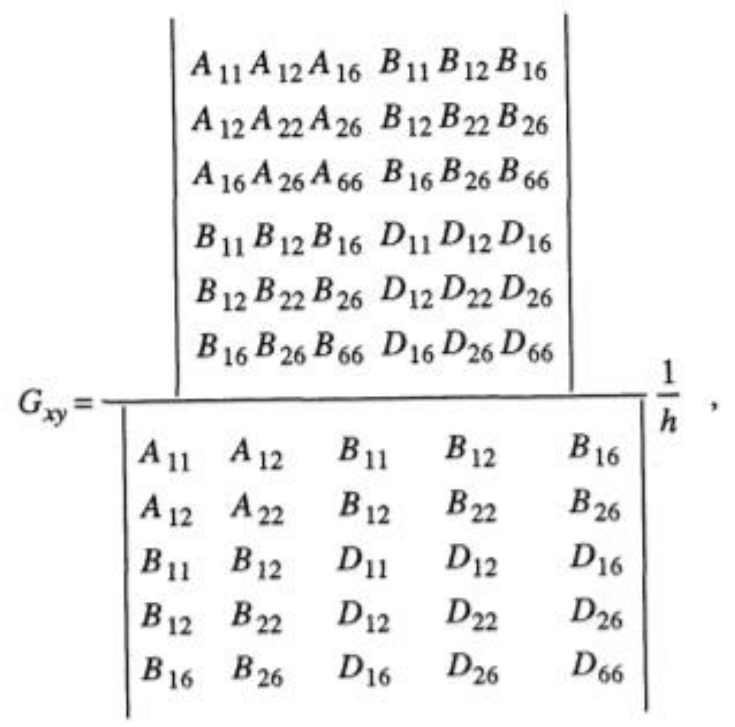$$
v_{x y}=\frac{\left|\begin{array}{lllll}
A_{12} & A_{26} & B_{12} & B_{22} & B_{26} \\
A_{16} & A_{66} & B_{16} & B_{26} & B_{66} \\
B_{11} & B_{16} & D_{11} & D_{12} & D_{16} \\
B_{12} & B_{26} & D_{12} & D_{22} & D_{26} \\
B_{16} & B_{66} & D_{16} & D_{26} & D_{66}
\end{array}\right|}{\left|\begin{array}{lllll}
A_{22} & A_{26} & B_{12} & B_{22} & B_{26} \\
A_{26} & A_{66} & B_{16} & B_{26} & B_{66} \\
B_{12} & B_{16} & D_{11} & D_{12} & D_{16} \\
B_{22} & B_{26} & D_{12} & D_{22} & D_{26} \\
B_{26} & B_{66} & D_{16} & D_{26} & D_{66}
\end{array}\right|}
$$ 


\section{Bibliography}

[1] H. T. Hong, H. J. Chun and H. S. Choi, Optimal strength design of composite bicycle wheels, vol. 15, International Journal of Precision Engineering and Manufacturing , 2014, pp. 16091613.

[2] I. Cycles, "Wheels," Intrepid cycles, [Online]. Available: http://intrepidcycle.com/cycletouring/building-a-touring-bike/wheels/.

[3] G. Drake and F. Zahradnik, "Carbon fiber (advantages of using carbon fiber in bicycle frames)," Bicycling, vol. 34, no. 8, p. 4, 1993.

[4] C. J. Burgoyne and R. Dilmaghanianj, "Bicycle Wheel as a Prestressed Structure," Journal of Engineering Mechanics, vol. 3, no. 119, pp. 439-455, 1993.

[5] U. Velo, "Carbon Bike Wheels: Advantages and Disadvantages," Ultimevelo, 7 June 2016. [Online]. Available: : https://www.ultimevelo.com/en/carbon-bike-wheels-advantagesdisadvantages/. [Accessed 1 March 2019].

[6] C. Meola, S. Boccardi and G. M. Carlomagno, "Infrared Thermography Basics," Infrared Thermography in the Evaluation of Aerospace Composite Materials, pp. 57-83, 2017.

[7] J. Brandt, The Bicycle Wheel, Palo Alto: Avocet, 2003.

[8] D. G. Wilson, J. Papadopoulos and F. R. Whitt, Bicycling Science, Cambridge, MA: MIT Press, 2004.

[9] A. S. Pippard and W. Francis, "XX On a theoretical and experimental investigation of the stress in a radially spoked wire wheel under loads applied to the rim," The London, Edinburgh and Dublin Philosophical Magazine and Journal of Science, vol. 11, no. 69, pp. 233-285, 1931.

[10] M. Hetenyi, "Beams on Elastic Foundation," Ann Arbor, Michigan, University of Michigan Press, 1956, pp. 156-163.

[11] H. Gavin, "Bicycle-Wheel Spoke Patterns and Spoke Fatigue," Journal of Engineering Mechanics, vol. 122, no. 8, pp. 736-742, 1996. 
[12] J. M. Minguez and J. Vogwell, "An analytical model to study the radial stiffness and spoke load distribution in a modern racing bicycle wheel," Proceedings of the Instituion of Mechanical Engineers, Part C: Journal of Mechanical Engineering, vol. 222, no. 4, pp. 563$576,2008$.

[13] M. Ford, J. M. Papadopoulos and O. Balogun, "Buckling of the bicycle wheel," in Bicycle Motion Dynamics Conference, 2016.

[14] N. Petrone and Giubilato, "Methods for evaluating the radial strucutre behaviour of racing bicycle wheels," in 5th Asia-Pacific Congress on Sports Technology, Padova, Italy, 2011.

[15] B. D. Agarwal, L. J. Broutman and K. Chandrashekhara, Analysis and performance of fiber composites, Hoboken, New Jersey: John Wiley and Sons Inc, 1990.

[16] T. J.-C. Liu and H.-C. Hu, "Fiber direction and stacking sequence design for bicycle frame made of carbon/epoxy composite laminate," Materials and Design, vol. 31, pp. 1971-1980, 2009.

[17] D. Liu, E. Lansing and L. E. Malvern, "Cracking in impacted glass/epoxy plates," Journal of Composite Materials, vol. 21, pp. 594-609, 1987.

[18] S. Abrate, Impact engineering of composite structures, Wien: Springer, 2011.

[19] J.-R. Cormier and G. LaPlante, "Study of the effects of low-velocity impact on a composite bicycle down tube," Composite Structures, vol. 198, pp. 144-155, 2018.

[20] T. B. Andersson, "On the effective consitutive properties of a thin adhesive layer loaded in peel," International Journal of Fracture , vol. 141, pp. 227-246, 2006.

[21] ASTM International, "ASTM D7136/D7136M-15 Standard Test Method for Measuring the Damage Resistance of a Fiber-Reinforced Polymer Matrix Composite to a Drop-Weight Impact Event," [Online].

[22] Union Cycliste Internationale, "UCI wheel approval proceudre," 23 December 2016. [Online].

[23] R. Usamentiaga, P. Venegas, J. Guerediaga, L. Vega, J. Molleda and F. Bulnes, "Infrared Thermography for Temperature Measurement and Non-destructive Testing," Sensors, vol. 14, no. 7, pp. 12305-12348, 2014. 
[24] C. Meola, S. Boccardi and G. M. Carlomagno, "Infrared Thermography Basics," Infrared Thermography in the Evaluation of Aerospace Composite Materials, pp. 57-83, 2017.

[25] H. Kaplan, Practical Applications of infrared thermal sensing and imaging equipment, Bellingham, Washington: SPIE Press, 2007.

[26] C. Meola, G. Carlomagno and F. Ricci, "Monitoring of impact damage in Carbon FIbre Reinforced Polymers," 2012 International Conference on Quantitative Infrared Thermography, 2012.

[27] R. Usamentiaga, "Real-time adapative method for noise filtering of a stream of thermographic line scans based on spatial overlapping and edge detection," Journal of Electronic Imaging, vol. 17, no. 3, 2008.

[28] R. Usamentiaga, C. Ibarra-Castanedo, M. Klein, X. Maldague, J. Peeters and A. SanchezBeato, "Nondestructive Evaluation of Carbon Fiber Bicycle Frames Using Infrared Thermography," Sensors, vol. 17, no. 11, 2017.

[29] N. Rajic, "Principal Component Thermography," DSTO Aeronautical and Maritime Research Laboratory, pp. 1-38, 2002.

[30] N. Rajic, "Principal Component Thermography for flaw contract enhancement and flaw depth characterisation in composite structures," Composite Structures, vol. 58, no. 4, pp. 521-528, 2002.

[31] K. E. Cramer and W. P. Winfree, "The Application of Prinicpal Component Analysis Using Fixed Eigenvectors to the Infrared Thermographic Inspection of the Space Shuttle Termal Protection System," Proceedings of the 2006 International Conference on Quanititative Infrared Thermography, 2006.

[32] C. Ibarra-Castenado, D. Gonzalez, M. Klein, M. Pilla, S. Vallerand and X. Maldague, "Infrared image processing and data analysis," Infrared Physics \& Technology, vol. 46, no. 1-2, pp. 75-83, 2004.

[33] Torayca Inc., "2510 Prepeg system Datasheet," [Online]. Available: https://www.toraycma.com/file_viewer.php?id=4856.

[34] S. Wijskamp, R. Akkerman and E. Lamers, "Residual Stresses Characterization of Laminated Composites Curing," in CCM14, twente, 2003. 
[35] NASA, "Fiber reinforced polymer composite material selection (GD-ED-2210)," NASA, 1996.

[36] U. Lehmann and W. Michaeli, "Preforming of fiber reinforced hollow components with the RTM/bladder molding process," Advanced composities; Proceedings of the 9th Annual ACCE Conference, pp. 531-541, 1997.

[37] U. Lehmann and W. Michaeli, "Cores lead to an automated production of hollow composite parts in resin transfer moulding," Composites Part A, vol. 29, no. 7, pp. 803-810, 1998.

[38] u. Lehmann and W. Michaeli, "Improved processing of resin transfer molding for the production of hollow parts with inflatable bladders," Proceedings of the 42nd International SAMPE Symposium and Exhibition, vol. 42, no. 1, pp. 13-23, 1997.

[39] D. M. Lane, J. J. Kutz, S. D. DeRoos and K. R. Alesse, "Comparison of processing techniques for the molding of hollow advanced composite parts," Proceedings of the 38th International SAMPE Symposium and Exhibition, vol. 38, no. 1, pp. 522-532, 1993.

[40] FLIR SYSTEMS, "FLIR SC5000 Series Manual," 2017. [Online]. Available: http://www.flir.co.uk/cs/display/?id=42577.

[41] FLIR Systems, "FLIR Tools/Tools+ User Manual," 2017. [Online]. Available: https://assets.tequipment.net/assets/1/26/FLIR_Tools_Plus_Manual.pdf.

[42] MathWorks Inc., MATLAB, MathWorks Inc., 2018.

[43] N. Salamon and R. Oldham, "Analysis for design of spoked bicycle wheels," Finite Elements in Analysis and Design, vol. 10, no. 4, pp. 319-333, 1992.

[44] A. Sasikumar*, J. Costa, D. Trias, E. Gonzalez, S. M. García-Rodríguez and P. Maimi, "Unsymmetrical stacking sequences as a novel approach to tailor damage," Composites Science and Technology, vol. 173, 2019.

[45] X. Sun, P. Qu, G. Liu, X. Yi, H. Su and Y. Jia, "Analysis on low velocity impact damage of laminated composite toughened by structural toughening layer," Polymer Compostes, vol. 38, no. 7, pp. 1280-1291, 2015.

[46] M. Ford, P. Peng and O. Balogun, "Acoustic Model testing of Bicycle Rims," Journal of Non-Destructive Evaluation, vol. 37, no. 16, 2018.

[47] A. Hartz, "Finite element analysis of the classic bicycle wheel," pp. 1-17, 2002. 
[48] D. Mariappan, S. Vijay and V. Ramamurti, "An efficient alogirthm for solving spoked wheels," Advances in Engineering Software, vol. 34, no. 1, pp. 25-30, 2003.

[49] M. Bruggeman, H. Sol and W. P. De Wilde, "Design and Production of FRP Bicycle Wheels," Reliability, Stress Analysis and Failure Prevention Aspects of Composite and Active Materials ASME, vol. 79, 1994.

[50] J.-M. Drouet and Y. Champoux, "A novel dynamometric hubset design to measure wheel loads in road cycling," in 8th conference of International Sports Engineering Association, Sherbrooke, Quebec, 2010.

[51] G. Gaussorgues, Infrared Thermography, London: Chapman \& Hall, 1994.

[52] F. Ahmad, H. S. Choi and M. K. Park, "A review: Natural Fiber Composites Selection in View of Mechanical, Light Weight and Economic Properties," Macromolecular Materails and Engineering, vol. 300, no. 1, pp. 10-24, 2014.

[53] W. Michaeli and U. Lehmann, "Combined moulding speeds hollow parts," Reinforced Plastics, vol. 40, no. 3, 1996.

[54] J. B., "Research and Development of composite sport goods in Marshal," International SAMPE Symposium and Exhibition, vol. 41, no. 1, pp. 379-392, 1996.

[55] Solidworks , Solidworks 2018, Dassault Systems, 2018.

[56] Hypermesh, Troy, Michigan: Altair Engineering Inc. , 2017.

[57] L. Lessard, J. A. Nemes and P. L. Lizotte, "Ulitization of FEA in the design of composite bicycle frames," Designer's Corner, vol. 72, no. 3, 1995.

[58] P. M. Sisneros, P. Yang and F. E. El-Hajjar, "Fatigue and Impact Behavior of Carbon Fibre Composite Bicycle Forks," Fatigue and Fracture of Engineering Materials \& Structures, pp. 672-682, 2011.

[59] J. T. H. a. T. M. C. P. O. Sjoblom, " Low velocity impact testing of composite materials," Journal of Composite Materials, vol. 22, pp. 30-52, 1988.

[60] S. Reddy, C. Yuvraj and P. K. Rao, "Design, Analysis, Fabrication and Testing of CFRP with CNF Composite Cylinder for Space Applications," International Journal of Composite Materials, 2015. 